\title{
Frontiers of light manipulation in natural, metallic, and dielectric nanostructures
}

\author{
E. De Tommasi ${ }^{1}$ - E. Esposito ${ }^{1} \cdot$ S. Romano ${ }^{1} \cdot$ A. Crescitelli ${ }^{1}$ - V. Di Meo ${ }^{1}$. \\ V. Mocella ${ }^{1} \cdot$ G. Zito $^{1} \cdot$ I. Rendina ${ }^{2}$
}

Received: 21 December 2020 / Accepted: 1 February 2021 / Published online: 18 March 2021

(c) The Author(s) 2021

\begin{abstract}
The ability to control light at the nanoscale is at the basis of contemporary photonics and plasmonics. In particular, properly engineered periodic nanostructures not only allow the inhibition of propagation of light at specific spectral ranges or its confinement in nanocavities or waveguides, but make also possible field enhancement effects in vibrational, Raman, infrared and fluorescence spectroscopies, paving the way to the development of novel high-performance optical sensors. All these devices find an impressive analogy in nearly-periodic photonic nanostructures present in several plants, animals and algae, which can represent a source of inspiration in the development and optimization of new artificial nano-optical systems. Here we present the main properties and applications of cutting-edge nanostructures starting from several examples of natural photonic architectures, up to the most recent technologies based on metallic and dielectric metasurfaces.
\end{abstract}

Keywords Nanophotonics · Natural photonic structures · Plasmonics · Dielectric nanophotonics $\cdot$ Metasurfaces $\cdot$ Advanced photonic sensors

\section{Introduction}

Light control and processing are dominating contemporary era, with applications involving telecommunications, imaging, sensing and biosensing, biomedicine, energy harvesting, and quantum computing, to name a few [1]. Technological progress

E. De Tommasi, E. Esposito, S. Romano contributed equally.

$\triangle$ I. Rendina

ivo.rendina@isasi.cnr.it

1 Consiglio Nazionale delle Ricerche, Istituto di Scienze Applicate e Sistemi Intelligenti, Sede Secondaria di Napoli, Via P. Castellino 111, Naples, Italy

2 Consiglio Nazionale delle Ricerche, Istituto di Scienze Applicate e Sistemi Intelligenti, Via Campi Flegrei 34, Pozzuoli, NA, Italy 
allowed the development of new materials and fabrication methods enabling light manipulation at the nanoscale, which in turn enlarged the range of optical functionalities and devices that can be designed [2,3]. Periodic modulation of refractive index in photonic crystals (PhCs) allows controlling the flux and the confinement of photons, which led to the realization of narrow-band filters, resonant mirrors, waveguides, waveguide splitters, optical swithches and nanocavities [4]. The strong light confinement which takes place in properly designed $\mathrm{PhCs}$ is able to enhance the interaction of radiation with an analyte, which inspired the development of novel optical sensing schemes [5,6]. Light fields can be modified and manipulated also by the interaction with metallic nanostructures supporting surface plasmons (local oscillations of plasma electrons), which led to the birth and growth of plasmonics [7]. The ability of plasmonic nanostructures to confine and enhance optical fields found applications in near-field scanning microscopy, non-linear optics, Raman spectroscopy, infrared spectroscopy, in the development of novel therapeutic treatments based on thermal effects and in the possibility to boost the efficiency of solar cells [8].

Taking into account the amount of time in which evolution acted on natural systems since the appearance of life on Earth, it is not surprising to observe the presence of more or less ordered optical nanostructures in several animals, plants and protists capable of light manipulation [9], mainly optimized for inter- and intra-species communication, camouflage or sunlight exploitation. Nature, for instance, already developed one-dimensional, two-dimensional and three-dimensional $\mathrm{PhCs}$ millions of years before the introduction and the mathematical formalization of artificial $\mathrm{PhCs}$, to name only one of the multitude of optical nanostructures exhibited by many biological systems, which still represent a source of inspiration in the development of novel designs in contemporary photonics [10].

Electromagnetic properties not observed in nature such as negative refraction, superimaging, invisibility, and gigantic chirality [11-14] can be achieved in metamaterials, artificial materials in which the optical properties depend not only on the material composition, but also on the interaction of fields with subwavelength, periodic or specially arranged inclusions (so called meta-atoms) [15]. A metasurface is a twodimensional array of meta-atoms which can be either metallic or dielectric scatterers or subwavelength apertures in a metallic or dielectric thin film [16]. We can thus distinguish between plasmonic metasurfaces, characterized by tight field confinement, broad bandwidth and small device footprint, and dielectric metasurfaces, not affected by the intrinsic optical losses typical of metallic nanostructures [17]. Being able to finely control the phase of incoming fields, metasurfaces found application in extraordinary reflection and transmission, in imaging as flat metalenses, in holography, in the observation of the optical spin Hall effect, and in the generation of optical vortex beams [18-21].

The present review article offers a wide overview of natural and artificial photonic nanostructures, starting from the main examples that can be found in living organisms and proceeding with the two main classes of artificial metasurfaces able to manipulate light at the nanoscale, metallic and dielectric ones, with particular attention devoted to field-enhancement effects and their application in sensing. 


\section{Photonic nanostructures in nature}

The first eye appeared during the so called Cambrian explosion, more than 500 million years ago, most likely in a very mobile predator [22]. Since then, light and color played a fundamental role in animal kingdom, both in intraspecies communication (e.g. for attracting mates and generally for sexual selection) and interspecies interaction (typically for camouflage or warning signs) [23,24]. Many algae and plants also developed external structures able to enhance photosynthetic efficiency [25] or to attract specific pollinators [26]. The availability of microscopic techniques with proper resolving power, mainly transmission and scanning electron microscopies, allowed the observation of more or less complex, ordered or quasi-ordered nanostructures at the basis of the optical response of several organisms, not necessarily related to the presence of pigments or materials with specific absorption in the visible spectral range. Some of the photonic nanostructures found in nature can cause coherent or incoherent scattering [27,28], others act as one-dimensional multilayer reflectors [29,30], polarization-selective reflectors [31,32], two-dimensional diffraction gratings [33,34] or can be shaped in even more complex hierarchical architectures whose optical characteristics often rely on a fine interplay between order and disorder [35-38].

In the following sections, the main photonic nanostructures present in nature are summarized, starting from the simplest multi-layered arrangements responsible for the so called structural color in many insects, fishes, birds, and flora up to architectures in which disorder or, conversely, hierachical organization of the ultrastructure plays a fundamental role in the resulting optical properties of the living system. Particular attention has been devoted to the description of the photonic properties of diatom frustules, which in recent years gained growing attention and for which a pletora of applications is repeatedly envisaged. In addition to some cases of direct exploitation of natural nanostructures in photonics and plasmonics, some examples of biomimetic devices are illustrated.

\subsection{Natural photonic crystals and structural color}

Probably the simplest photonic nanostructure in biological systems can be found in iridoviruses [22,39]. Even though, as all viruses, they cannot be classified with no ambiguity as living organisms [40], they are able to self-assemble in paracrystalline arrays inside the infected cell, causing optical iridescence arising from multiple Bragg scattering (see Fig. 1). Viral colloidal assemblies derived from insect-specific Wiseana iridescent virus have been obtained in vitro by sedimentation and flow-assisted thinfilm assembly [39], showing optical characteristics in the visible spectrum. Since virus particles are intrinsically monodisperse with highly defined simmetries, the polydispersity index in size and mass of the viral colloids nearly equals 1 . Viruses find application also in plasmonics, e.g. as core substrates in the fabrication of metallodielectric nanoparticles. For example the surface charge of capsids of chilo iridescent viruses can be decreased by controlled introduction of excess electrolyte, and the subsequent attachment of gold nanoparticles allows obtaining plasmonic nanoshells with resonances in visible and near infrared spectral regions [43]. The use of viruses thus 


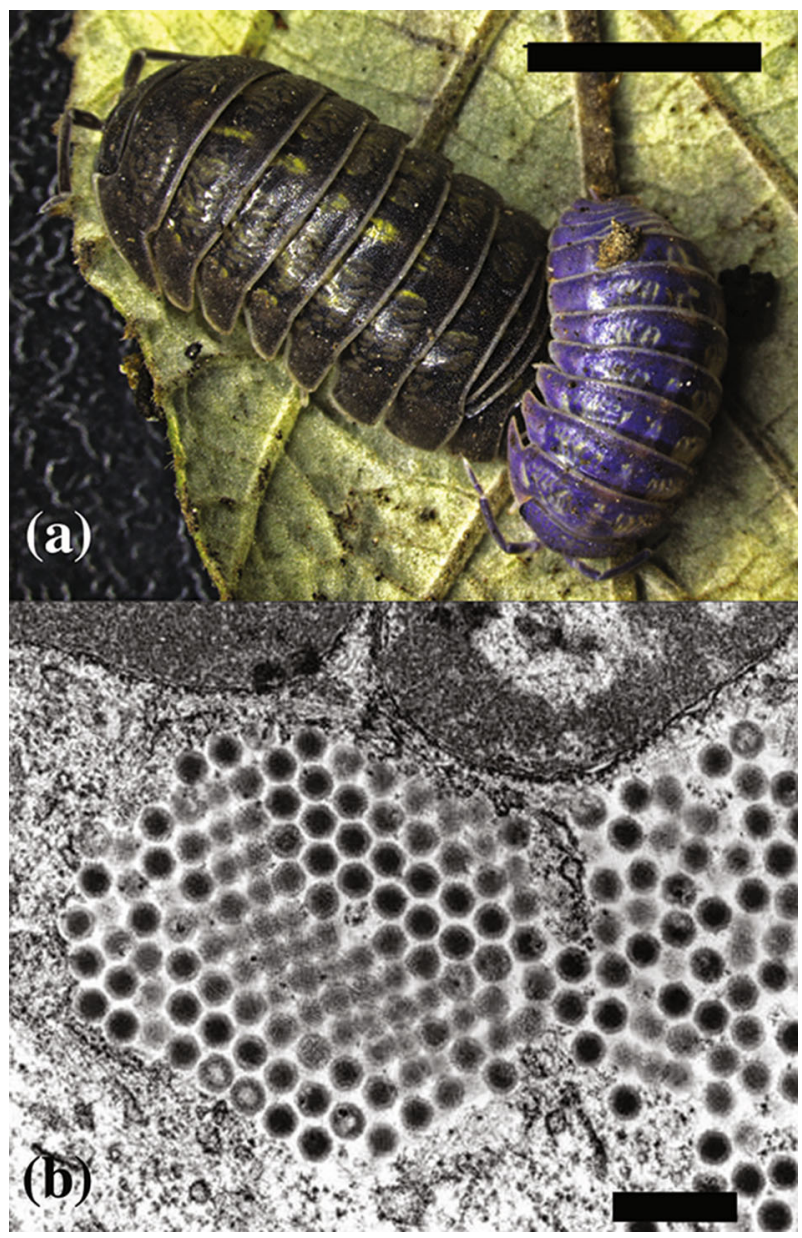

Fig. 1 a An Armadillidium decorum isopod infected by iridovirus in an advanced stage is characterized by blue coloration due to Bragg scattering from regularly self-assembled viral particles (scale bar: $1 \mathrm{~cm}$ ). Reproduced with permission from [41]. b Transmission electron micrograph of a paracrystalline array of iridoviruses cultured from the liver of a Rana temporaria frog (scale bar: $400 \mathrm{~nm}$ ). Reproduced with permission from [42]

potentially provide cores with smaller diameters than currently used silica (even below $80 \mathrm{~nm})$.

Quasi-ordered periodic nanostructures (mainly in the form of multilayer arrangements) acting as selective reflectors are also diffused as constitutive parts of many animals and flora [44]. They are at the basis of the so called structural color, which does not depend on pigmentation but mainly on the geometry and the refractive index contrast of these structures with respect to the environment [45]. In addition to multilayer interference, other phenomena such as coherent scattering and diffraction contribute to structural coloration and iridescence. The combination of these effects with specific absorption from pigments and irregularity on different length scales of the system 
increases the intensity of the whole optical effect and broadens the angular range of the overall reflectance [23].

Multilayer reflection is extremely frequent in beetles and originates from a stack of layers of the appropriate thickness alternating in refractive index. For layers of alternating optical thicknesses $n_{1} d_{1}$ and $n_{2} d_{2}$, the reflectance peak wavelength is given by the well known interference condition:

$$
\lambda_{\max }\left(\theta_{0}\right)=2\left(n_{1} d_{1} \cos \theta_{1}+n_{2} d_{2} \cos \theta_{2}\right)
$$

with $\theta_{0}$ angle of light incidence for the first layer, and $\theta_{1}$ and $\theta_{2}$ angles at the interfaces of the multilayer. In the japanese jewel beetle Chrysochroa fulgidissima (see Fig. 2ac), for example, the different colors of the elytron (green), the ventral area (orange), and the elytron stripes (purple) are originated by interference from alternating layers of chitin and melanin whose spacing varies in different locations [46]. The refractive index does not change abruptly from layer to layer, smoothly oscillating between about 1.6 and 1.7 with a periodicity depending on the site. Finally, a melanin substrate fully absorbs all the light backscattered from the deep layers of the cuticle, enhancing the intensity and purity of the structured color.

The architectures originating structural colors can be rather more complex, as can be seen for the Lamprocyphus augustus weevil (see Fig. 2d-g). Each single scale of the exoskeleton is composed by nanostructured domains of ordered air holes in a surrounding cuticular matrix. These domains are characterized by the same crystal lattice ( $\simeq 300 \mathrm{~nm}$ ) but different orientations, thus leading to a near angle-independent coloration [47]. The combined use of focused ion beam (FIB) milling and SEM imaging allowed reconstructing the three-dimensional, diamond-based structure of the domains and the corresponding photonic band diagrams. The proximity and overlap of three stop gaps in the $\Gamma-W(210), \Gamma-K(110)$, and $\Gamma-X$ (100) directions cover the whole green spectral region, thus explaining the intense angle-independent coloration of the scales.

Probably the most studied hierarchical photonic architecture able to produce intense structural coloration can be found in several species of the Morpho butterfly [35,36,49]. Due to the combination of ordered and disordered structures, it gives rise to several, interacting coherent and incoherent optical effects, first of all the multilayer interference originating from the lamellae of the "tree-like" ridges observed in the single scales of the wings (see Fig. 3a-c). The presence of multiple layers of scales, their different angular orientation, the different height of the individual ridges and the presence of an absorbing background contribute to the overall visual appearance of these butterflies [50], characterized by flashing, intense blue wings mainly used by males to mark their territory and scare off potential rivals in mating. In particular, the length distribution of the ridges and the width of each single ridge control the angular width of the reflection cone in the compressed and extended directions, respectively (see Fig. 3d), while the distribution of height of the ridges suppresses the diffraction grating effect typically obtained from periodic structures, giving rise to a smooth, uniform angular reflection $[45,50]$. It has been shown that the reflectance spectra of nanostructured scales of Morpho sulkowskyi butterfly are affected by the nature and concentration of the vapours to which the scales are exposed [51]. This dependence is mostly due to physical adsorp- 
(a)

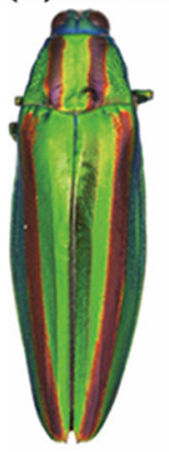

(b)

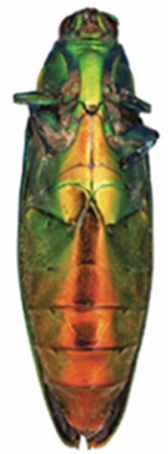

(c)
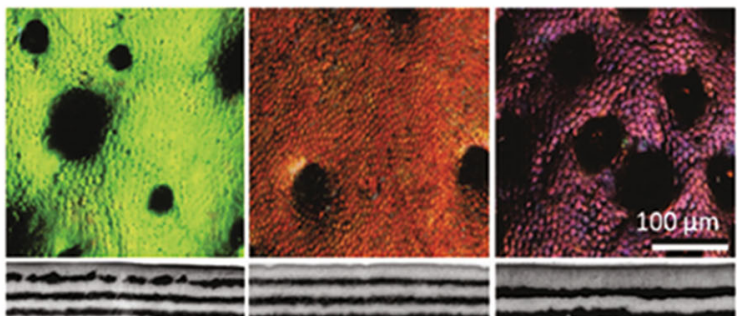
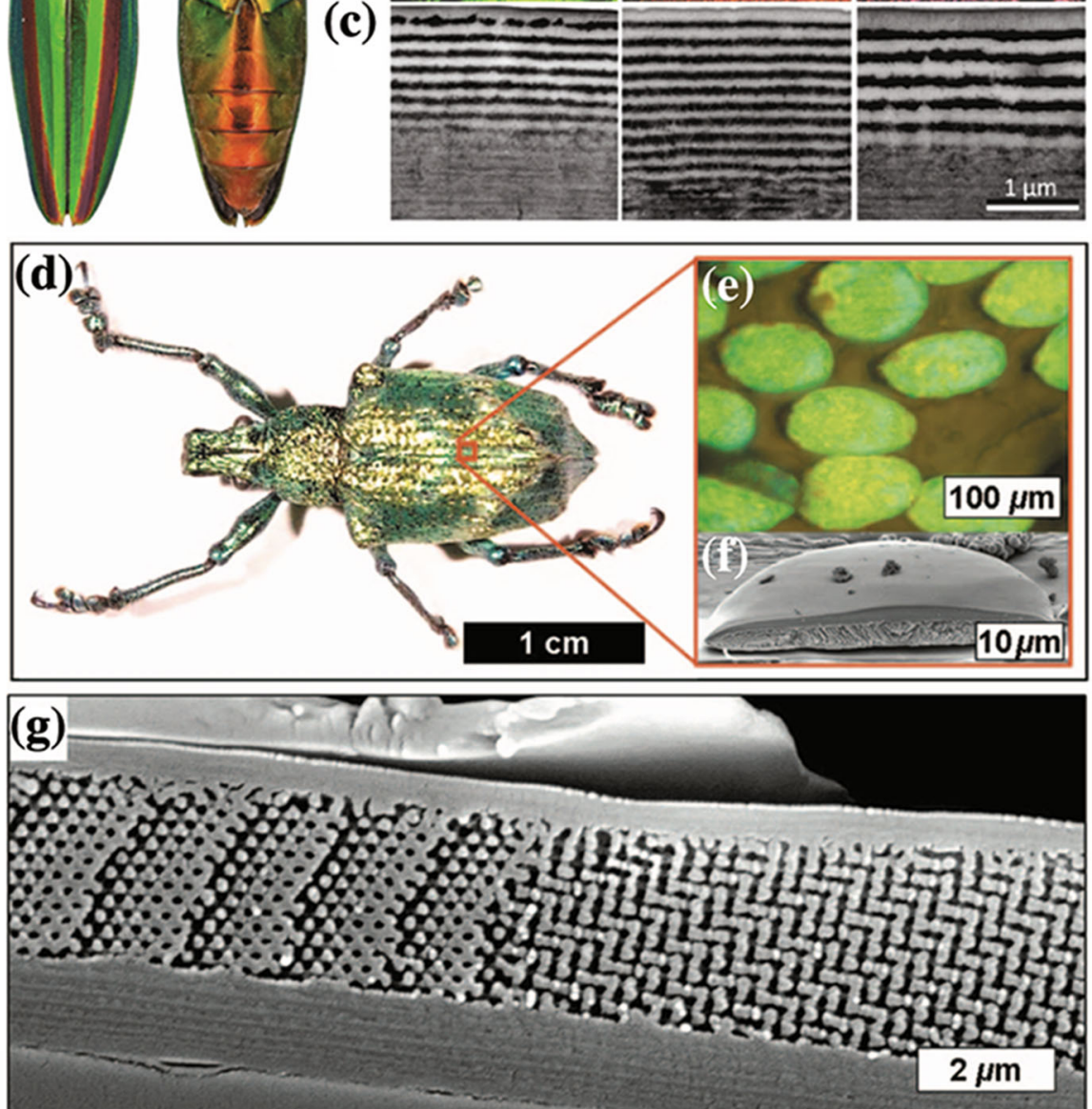

Fig. 2 a Japanese jewel beetle Chrysochroa fulgidissima. Microscopy (b) and TEM (c) images of $C$. fulgidissima cuticles in different sites. d Lamprocyphus augustus beetle. Optical (e) and (f) SEM images of individual scales. $\mathbf{g}$ SEM image of the cross-section of a single scale. a-c Reproduced with permission from [46]. d-g Reproduced with permission from [47]

tion and capillary condensation of vapours in the nano-sized domains of the scales. The application of principal component analysis (PCA) to the differential reflectance spectra obtained under exposure to vapours with similar solvent polarities and refractive indices (water, methanol, and ethanol) allows to differentiate between them, thus demonstrating the possibility to use Morpho nanostructured scales as efficient, selective chemical sensors. Properly metallized butterfly scales (or their artificial, metallic 
(a)

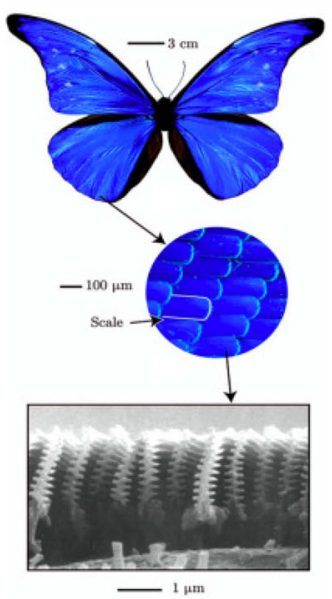

(b)

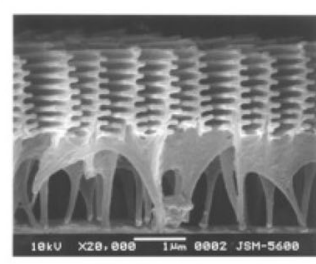

(d)

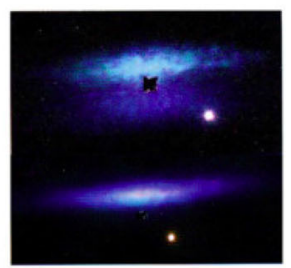

(c)

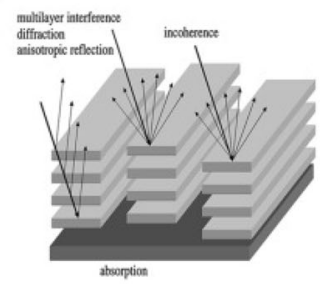

(e)

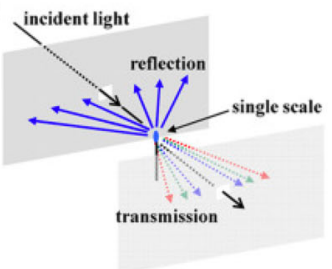

Fig. 3 a Images of a Morpho rhetenor butterfly: dorsal side (top), detail of a wing showing scales (middle), and SEM image showing the section of a single scale with its characteristic ridges. Reproduced with permission from [48]. b Ultrastructure of ground scales of M. sulkowskyii. $\mathbf{c}$ Schematic description of the effects contributing to the overall optical response. $\mathbf{d}$ Reflection patterns of a single cover scale (up side) and of a single ground scale (down side) of $M$. didius. e Experimental scheme for the detection of the reflection and transmission patterns of a single scale. b-e Reproduced with permission from [45]

replicas) have been successfully employed in plasmonics [52]. As an example, Garrett et al. [53] obtained efficient surface enhanced Raman spectroscopy (SERS) substrates by thermal evaporation of gold or silver onto the conical, cuticular nanostructures found on the wings of Graphium weiskei butterfly. Both these bio-substrates and their replicas obtained by reactive ion etching were characterized by enhancement factors of the order of $10^{6}$ (for gold) and $10^{7}$ (for silver). Furthermore, they have been successfully exploited in avidin-biotin assay experiments, reaching sensitivities comparable with those of a typical enzyme-linked immunosorbent assay (ELISA) system.

\subsection{Helicoidal structures and polarized light}

In some synthetic systems such as chiral nematic liquid crystals, the lack of inversion symmetry of the constituent molecules induces the formation of small azimuthal twists which in turn causes strong circularly polarized color reflections [31]. Similar helicoidal structures (in this case based on chitin fibres arranged in birefringent layers) can be observed in the elytra of several beetle species [49,54]. An example is given by the exocuticle of the beetle Chrysina gloriosa, which selectively reflects left circularly polarized light (see Fig. 4a, b) [32,55]. The microstructure of the beetle surface is organized in a near-hexagonal packing of conical-protruded cells resembling the spiraled conic domains that arise on the free surface of a siloxane oligomer-based cholesteric liquid crystal (see Fig. 4e, f and Ref. [56]). Since the pitch $p$ of the helicoidal structure is of the same order of magnitude of the wavelength of visible radiation, Bragg-like, polarization-dependent reflection takes place. For an angular incidence $\phi$ and for an 


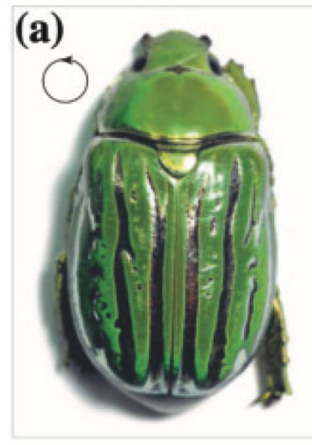

(c)

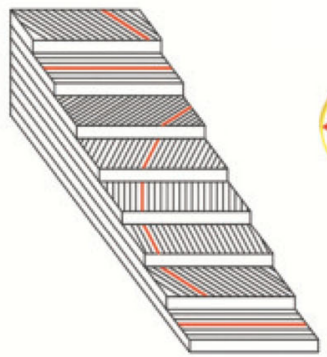

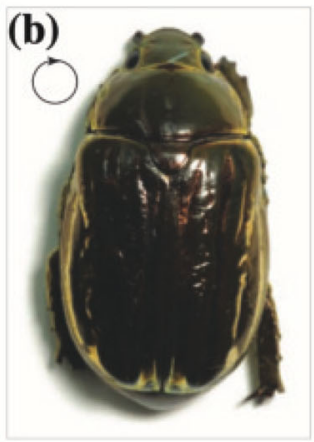

(d)

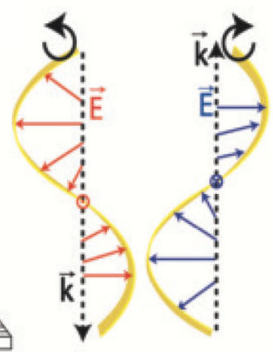

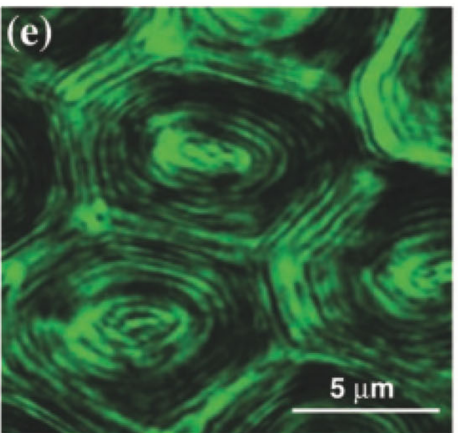

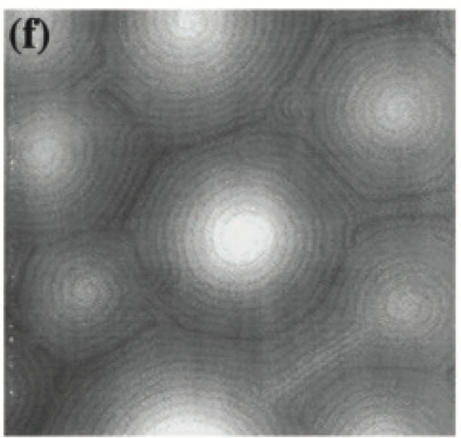

Fig. 4 C. gloriosa beetle as seen in unpolarized light (or with a left circular polarizer) (a) and with a right circular polarizer (b). Left-handed helicoidal structure (c). Right-handed (transmitted) and left-handed (reflected) circular polarized light with indication of the wavevector $\mathbf{k}$ (d). Microstructure of cellular pattern of $C$. gloriosa as obtained by laser scanning confocal microscopy (e). AFM image of the spiraled, focal-conic domains at the free surface of a siloxane oligomer-based cholesteric liquid crystal (f). a, b, and e Reproduced with permission from Ref. [32]. $\mathbf{c}$ and $\mathbf{d}$ Reproduced with permission from Ref. [57]. f Reproduced with permission from Ref. [56]

orientation of the helical axis $\theta$ we have:

$$
\lambda_{0}=n p \cos (\phi-\theta)
$$

with $\lambda_{0}$ wavelenght of the peak of the reflected light and $n$ average refractive index. The spectral width $\Delta \lambda$ of the reflection peak is related to the birefringence $\Delta n=n_{o}-n_{e}$, with $n_{o}$ and $n_{e}$ refractive indeces for polarization perpendicular and parallel to the axis of anisotropy, respectively. The reflectance of $C$. gloriosa spans the interval from 500 to $600 \mathrm{~nm}$ with peaks at 530 (green) and 580 (yellow) nm. The strong circularly polarized reflection produced by $C$. gloriosa exocuticle is likely related to intraspecies communication, as it has been verified for the marine crustacean Odontodactylus sp. [31]. 


\subsection{Blackness, whiteness and transparency in nature: the role of disorder}

In most insects, temperature is mainly regulated by the controlled absorption of solar radiation. Furthermore, the brightness and visual appearance of many colored insects is strongly enhanced by the darkness of the frame that surrounds their colored regions (see Fig. 5a). Blackness in natural systems is not uniquely associated with the presence of broad-band absorbing pigments, since other effects can contribute to the final appearance. In Papilio ulysses butterflies, for example, disordered, aperiodic nanowalls present in their wing scales (see Fig. 5a) scatter incident radiation towards the interior of the structure, increasing the optical path length throughout the diffusely distributed pigmentation of the substrate [58], resulting in a remarkable lowering of back-reflection. Wang et al. [59,60] studied, by means of finite difference time domain (FDTD) method, the omnidirectional absorption induced on incident optical radiation by a disordered nano-hole structure with ridges inspired by $P$. ulysses, proving its stability at different polarizations and wavelengths.

In absence of pigments, multiwavelength scattering of light induced by aperiodic and multiply oriented interfaces can give rise to high level of whiteness, brightness and brilliance, as in the case of Cyphochilus sp. or Lepidiota stigma beetles, whose scales are composed by an intricate network of cuticular nano-filaments (see Fig. 5b) [27,28]. In the case of Cyphochilus sp., in particular, two-dimensional fast Fourier transform (FFT) analysis of TEM images of the nanostructures and the examination of light diffracted by individual scales, confirmed the absence of well-defined periodicities [27]. The combined effect of the system aperiodicity, high void fraction and refractive index contrast ( $\Delta \mathrm{n} \simeq 0.56$ ) allows obtaining high values of whiteness (60) and brightness (65) even if the scales are only $5 \mu \mathrm{m}$ thick.

Omnidirectional anti-reflection behaviour is observed in the transparent regions of the wings of Greta oto, not accidentally known as glasswing butterfly (see Fig. 5c). This feature has been attributed to the presence of nano-pillars characterized by a disordered spatial arrangement and a random height and width distribution [62]. The ring-shaped, two-dimensional Fourier power spectrum of the top-view SEM images of the nanostructures confirms the quasi-random positioning of the pillars and the presence of isotropic order only on a long-range scale. The combined application of effective medium theory and transfer matrix method to randomly placed nanopillars characterized by a gaussian distribution of heights and widths allowed the reproduction of reflection and transmission spectra of the structure for a given polarization and for all angles of incidence, demonstrating low values of reflectance even for large view angles (up to $80^{\circ}$ ). Possible applications include the large-scale production of efficient anti-reflective surfaces improving the light collection in solar cells or for efficient light extraction in light-emitting diodes.

\subsection{Living photonic crystals: the case of diatoms}

The first organisms deserving the explicit classification of "living photonic crystals" have been diatoms [63]. Diatoms are ubiquitous, unicellular microalgae constituting the major component of phytoplancton and inhabiting all seas and freshwaters [64]. It 
(a)
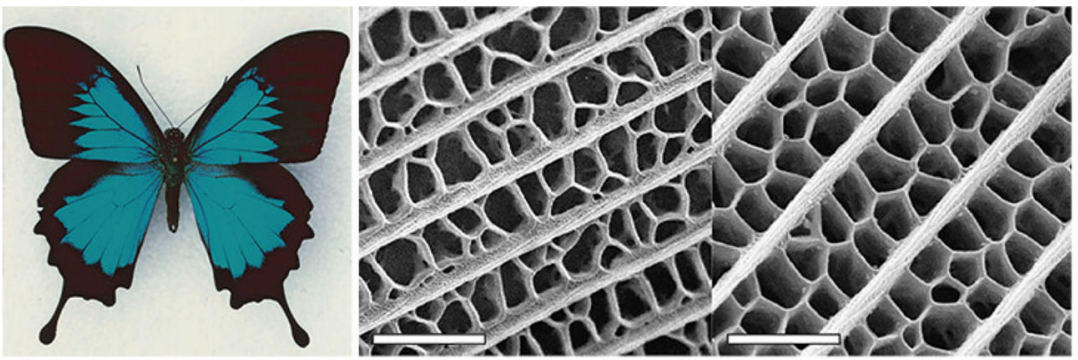

(b)
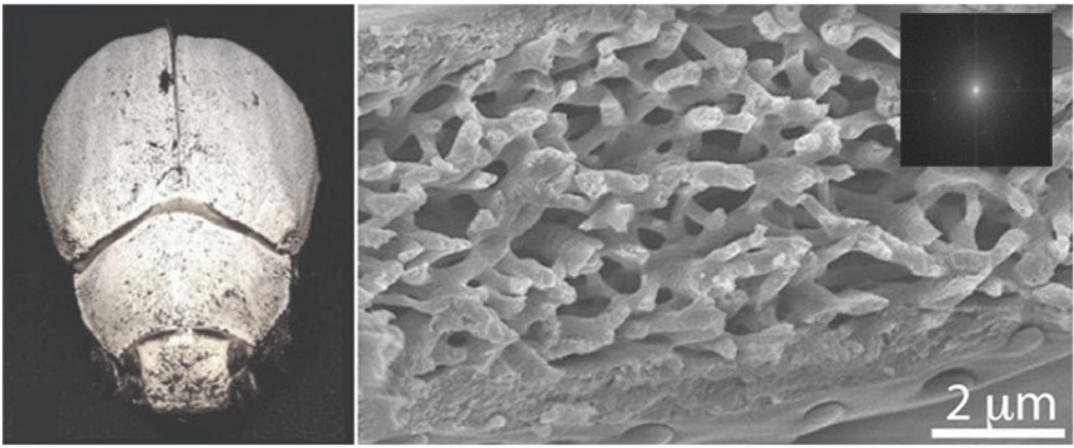

ezavant. The refleqtance, however, depenc larization when the angle of incidence is gure $7 a, b$ show how the reflectance for 500 pends on the angle of incidence for differe ights, that is for $T^{\prime}(s-)$ nolarized and

(c)

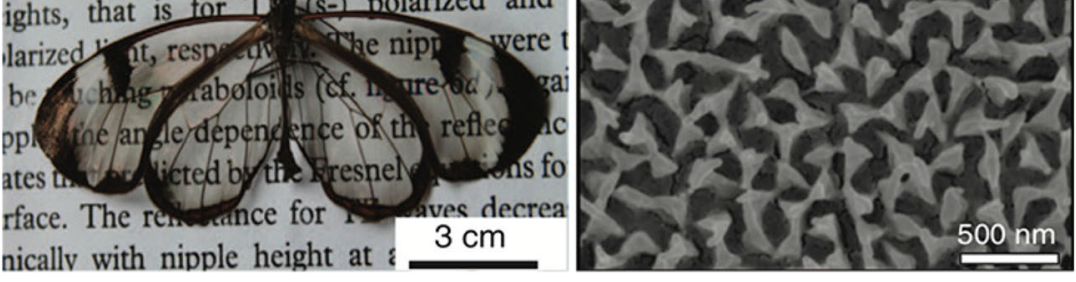

Fig. 5 a $P$. ulysses butterfly and SEM images of the disordered nanostructure of a single black scale from the lustrous (left) and matt (right) black regions. Scale bars: $3 \mu \mathrm{m}$ and $2 \mu \mathrm{m}$, respectively. Reproduced with permission from Refs. [61] and [58]. b Cyphochilus sp. beetle (left) and SEM image of the random network of cuticular filaments responsible of its whiteness (right). In the inset, the result of two-dimensional FFT of a TEM image (not shown) of the scale interior showing the absence of any well-defined periodicity. Reproduced with permission from Ref. [28] and from the Supporting Material of Ref. [27]. c G. oto butterfly (left) and SEM top view of the quasi-randomly positioned pillars found in the transparent regions of its wings (right). In the inlet, the two-dimensional Fourier power spectrum of the SEM image is shown, with a ring-shaped distribution caused by the disordered arrangement of the pillars. Reproduced with permission from Ref. [62] 
(a)

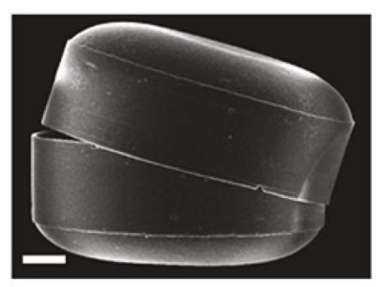

(b)

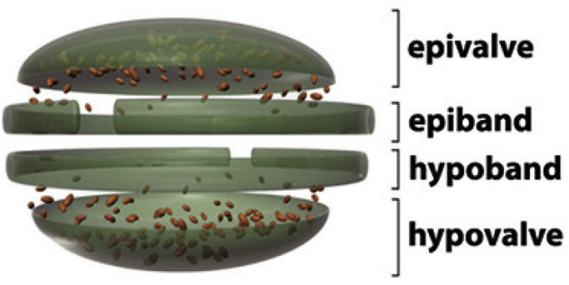

(c)

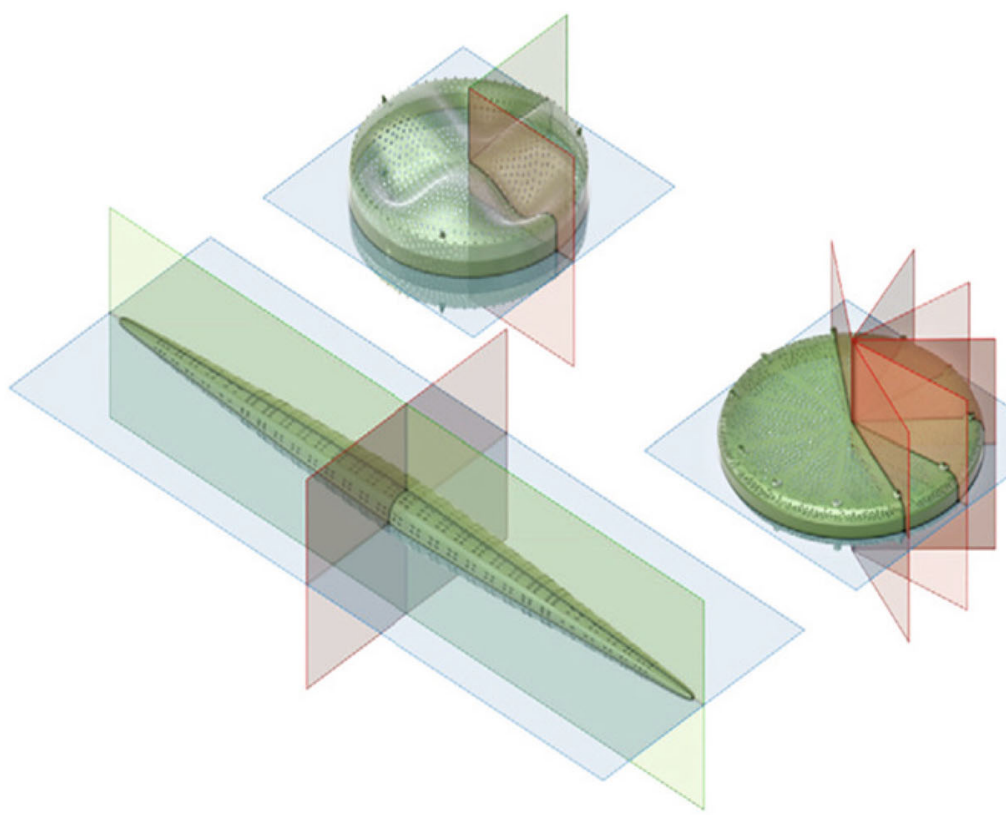

Fig. 6 a Frustule of a centric diatom, with an epitheca overlapping a hypotheca in a petri-dish-like structure. Scale bar: $50 \mu \mathrm{m}$. Reproduced with permission from Ref. [66]. b Scheme of a generic frustule with indications of the single components of the techae. Chloroplasts inside the frustule are also shown. Adapted with permission from Ref. [67]. c Schematic representations of pennate and centric diatom frustules, with indication of the corresponding simmetry planes. Reproduced with permission from Ref. [37]

is estimated that about $20 \%$ of global primary production is to be ascribed to the photosynthetic activity of diatoms [65]. About $10^{5}$ species of diatoms have been classified so far, differing in dimensions (with sizes ranging from some tens of microns to 1-2 millimeters) and morphology but sharing a common feature, i.e. the presence of a hydrated, micro- and nano-structured silica shell termed frustule.

Frustules consist of a epitheca overlapping a hypotheca in a petri-dish-like shape (see Fig. 6a, b). Every theca is constituted by a valve and a series of lateral bands (girdle bands or copulae). Valves and girdles are ornate with regualar, periodic patterns of micro- and nano-pores, whose dimensions range from some tens of nanometers to about 1-2 microns, depending on the considered species and on the their location in 

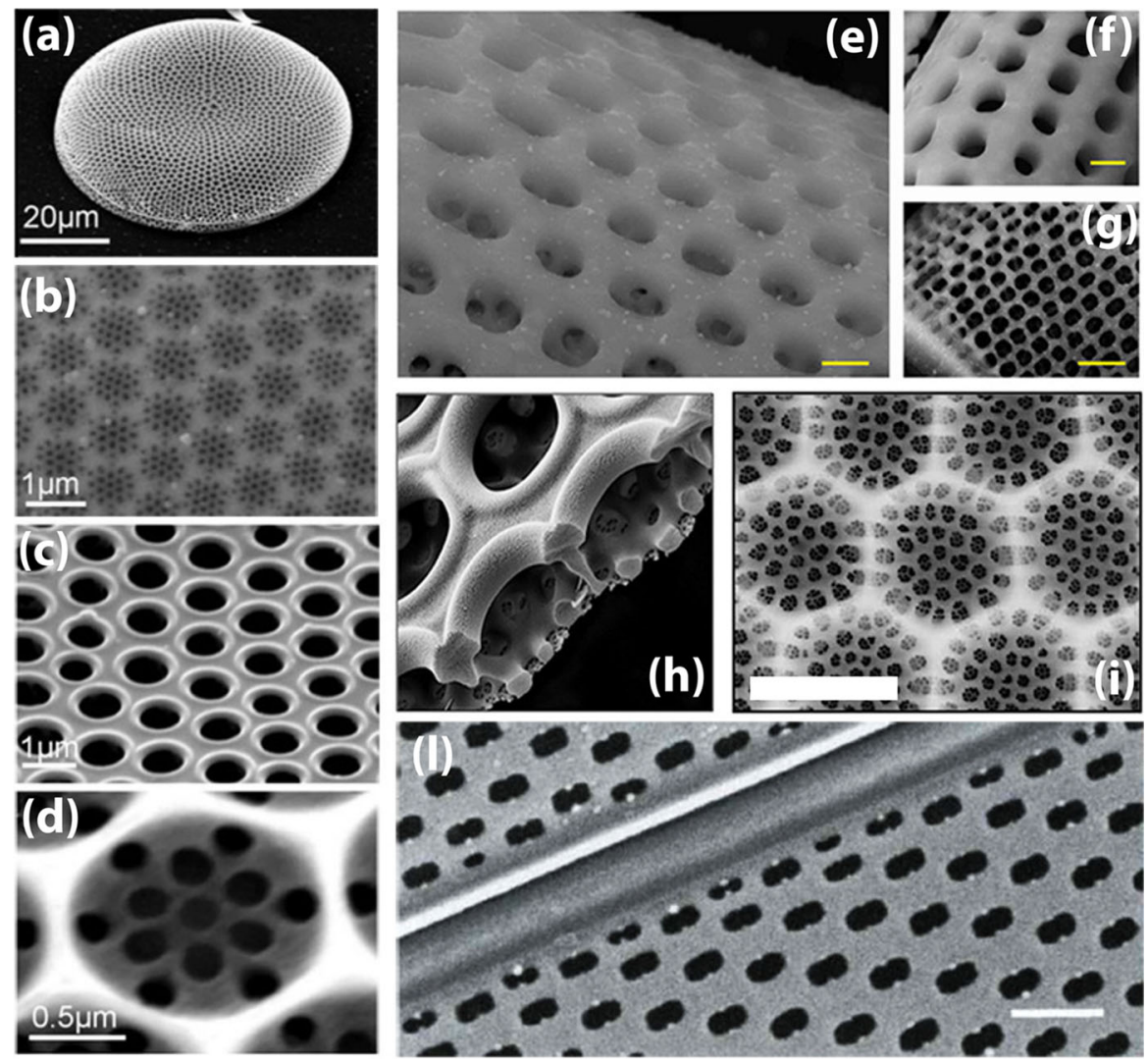

Fig. 7 Examples of pore patterns in diatom frustules. External plate (a), cribrum (b), foramen (c) and cribrum detail (d) of a Coscinodiscus sp. valve. Reproduced with permission from Ref. [74]. (e)-(g) pore patterns in diatom shells from diatomaceous earth (sedimentary powder derived by fossilized diatoms). Scale bars: $100 \mathrm{~nm}$ (e), $150 \mathrm{~nm}$ (f) and $400 \mathrm{~nm}$ (g). Reproduced with permission from Ref. [75]. Detailed sectional (h) and top (i) view of a centric diatom valve. Scale bar: $1 \mu \mathrm{m}$. Reproduced with permission from Ref. [76]. I Detail of the external valve of Pleurosigma acus (stage in morphogenesis). Scale bar: $1 \mu \mathrm{m}$. Reproduced with permission from [77]

the frustule (see Fig. 7). In particular, valves are structured in a series of overlapped layers with pores of distinct dimensions and lattice constants (cribellum, cribrum and foramen, see Fig. 7a-d). According to frustule symmetry, diatoms can be distinguished in centric (mainly planktonic and with a radially symmetric frustule) and pennates (mostly benthic and provided with a bilaterally symmetric frustule). Frustule functions comprise mechanical stability, being able to resist to pressures ranging from 1 to $7 \mathrm{~N} / \mathrm{mm}^{2}$ (equivalent to $100-700 \mathrm{t} / \mathrm{m}^{2}$, as experimentally verified in Ref. [68]); sorting of nutrients (such as $\mathrm{NH}_{4}^{+}, \mathrm{HCO}_{3}^{-}, \mathrm{NO}_{3}^{-}$etc.) from harmful agents (e.g. bacteria and viruses) [69,70]; reduction of sinking speed [71]; finally, optimization of coupling with sunlight [72]. Several optical properties of frustules were known since the early 1970s [73]: if observed in the proper azimuth and in a dark background, diatom frustules present brilliant colors due to diffraction induced by their ornamentations; the frustule 

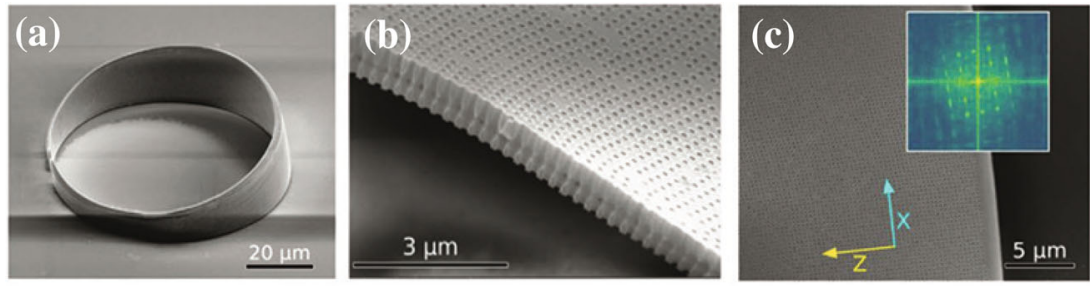

(d)

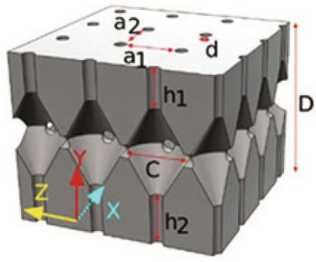

(e)

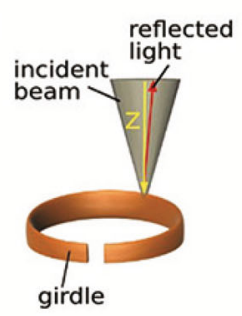

(f)

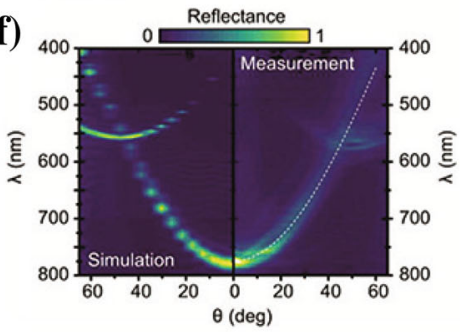

Fig. 8 a $C$. granii girdle. b Detail of the bilayered girdle provided with a square-lattice of holes and chambers. c Square lattice of the girdle and the result of its FFT analysis. d CAD of four unit cells of the girdle reconstructed from its SEM morphological characterization. $a_{1}=285 \pm 5 \mathrm{~nm} ; a_{2}=279 \pm 11 \mathrm{~nm}$; $d=124 \pm 21 \mathrm{~nm} ; h_{1}=225 \pm 21 \mathrm{~nm} ; h_{2}=250 \pm 32 \mathrm{~nm} ; C=290 \pm 18 \mathrm{~nm} ; D=745 \pm 43 \mathrm{~nm}$. e Scheme of the experimental setup used to reconstruct the photonic band structure of the girdle. $\mathbf{f}$ FDTD numerical results (left) and experimental results (right) relative to the reflectance as a function of the incidence angle and wavelength for a girdle immersed in water. Reproduced with permission from Ref. [78]

of several diatom species presents optical activity, being able to change the state of polarization of the incident radiation; some diatoms of the genus Actinocyclus switch from one color distribution to the complementary one as the mode of illumination changes from bright field to dark field.

In 2004 Fuhrmann et al. numerically reconstructed the photonic band structures of valves and girdles of a Coscinodiscus granii frustule, modeled as photonic crystal slab waveguides [63]. Valves and girdles present, for this species, a hexagonal and a square pattern of sub-micrometric holes, respectively. Furthermore, the authors evaluated the efficient optical coupling of the chloroplasts, the photosynthetic units of the cell, with the evanescent waves associated to the guided modes. Chloroplasts indeed tend to locate close to the frustule walls under dim lighting conditions, thus well within the evanescent field. The photonic properties of $C$. granii girdles seen as photonic crystal slabs have been further deepened by Goessling et al. [67,78] by means of an accurate SEM morphological characterization, 3D numerical simulations based on FDTD calculations and measurements of their photonic properties in in-plane direction by means of microscatterometry (see Fig. 8), both in air and in water. These studies showed the presence of a pseudogap, i.e., a photonic band gap that only exists for particular directions of propagation. When the girdle is immersed in water, the pseudo gap appears in the near-IR for normal incidence and tends to be blue-shifted as the incidence angle $\theta_{i}$ is increased (see Fig. 8f). Also waveguiding of green light, already suggested by Fuhrmann et al. [63], has been experimentally observed. Since both the pseudogap and the waveguided modes do not resonate in the spectral range where the 
efficiency of photosynthesis is high, the authors speculate that the photonic properties of the girdle are such to reduce the interference of photosynthetically not productive wavelengths with pigment light absorption.

The incorporation of rhodamines in the silica matrix of $C$. granii and Coscinodiscus wailesii frustules by means of metabolic doping allowed to study how the frustule photonic structure affects the photoluminescence emission of the embedded dyes [79], in analogy with the study of the interaction between a photonic crystal and an integrated emitter (e.g. a quantum dot [80]), which is fundamental for the development of new light sources based on hybrid materials. The possibility to metabolically insert semiconducting or metallic materials in diatom frustules during the cell growing process (experimentally demonstrated, for example, in Ref. [81] and [82]) can induce, as numerically evaluated in Ref. [83] for $C$. wailesii valves doped with titania, the appearance of complete photonic band-gaps with gap-midgap ratios $\Delta \omega / \omega_{0}$ up to $6.5 \%$.

Yamanaka et a. [84] calculated the dispersion relations of the modes supported by the inner shell of a Melosira variance diatom (provided with pores arranged in a triangular lattice), considering air and water as background. In both cases they observed that, in the spectral range between 400 and $500 \mathrm{~nm}$ and in $M-K$ direction, the dispersion curves are parabolic, which implies an enhanced interaction between light and the shell seen as a photonic crystal slab. The authors hypothesize that this behavior mechanism could be exploited by the diatom to weaken a possible excess supply of blue light which, above a specific threshold, induces the production of active oxygen (harmful for the cell).

The use of a fiber-based supercontinuum source emitting coherent optical radiation from $400 \mathrm{~nm}$ to beyond $1700 \mathrm{~nm}$ allowed the analysis of light transmitted by a single C. wailesii valve over a wide spectral range at different locations in the porous plate [85]. The transmission spectra acquired after probing different regions of the valve (corresponding to slightly different average pore spacing) present dips at different wavelengths. In particular, moving from the center of the valve towards the edge, the dip tends to shift toward longer wavelengths (as confirmed by the observations of the colors of the transmitted diffraction patterns), following the increasing spacing of the pores characterized by a hexagonal arrangement.

The application of 3D FDTD calculations and scanning near-field optical microscopy (SNOM) to valves of Nitzchia filiformis diatom revealed the presence of resonances corresponding to the absorption spectrum maxima of chlorophyll $\mathrm{A}$, the predominant photosynthetic pigment in diatoms [86,87]. It is hypothesized that the frustule acts both as a grating-coupler and a photonic crystal slab, enhancing the collection of photosynthetic active radiation.

It has been demonstrated that the valves of several species of centric diatoms are able to collect and confine optical radiation, acting as microlenses. In 2007 De Stefano et al. [88] verified that a single $C$. wailesii valve is able to focus coherent radiation at $785 \mathrm{~nm}$ in a $10 \mu \mathrm{m}$ wide spot at a distance of about $100 \mu \mathrm{m}$ away from the valve itself. The phenomenon has been ascribed to coherent superposition, along the optical axis, of the diffraction contributions coming from the single pores of the valve and it has been confirmed, both experimentally and numerically and also for incoherent visible radiation, for the same species [89] and for Arachnoidiscus ehrenbergii [90], Cos- 
cinodiscus centralis [91], and C. granii [92] single valves. The position of the light spots along the optical axis depends on the wavelength of the incoming radiation. Indeed the divergence angle of the light diffracted by a single pore is proportional to wavelength, thus, the longer the wavelength, the closer to the valve the focused spots take place. This means that, for sufficiently short wavelengths, the light confinement occurs quite far from the valve or does not take place at all $[89,90,93]$. This could represent a mechanism by which diatoms are able to efficiently collect photosynthetic active radiation and, simultaneously, protect themselves from harmful ultraviolet radiation [93]. Other mechanisms potentially involved in UV-shielding are absorption by frustule silica and UV-visible wavelength conversion induced by nanostructured silica photoluminescence [72]. The confinement of optical radiation happens even closer to the valve, thus inside the cell, when considering water as the environment in which the valve is immersed, as numerically demonstrated in Ref. [89] and derived from digital holography measurements in Ref. [94]. The digital hologram reconstruction procedure allows indeed the retrieval of both amplitude and phase of the optical field which interacts with the valve at every position along the optical axis and for any value of the refractive index of the surrounding environment.

\subsubsection{Applications of diatom frustules: some examples}

Diatom cultures represent a sort of living, low-cost, large-scale nanofactories able to provide complex, three-dimensional nanostructures hardly feasible even by the most recent lithographic techniques with high reproducibility and rates. Far from aspiring to cover all the achieved and envisaged uses of diatom frustules in nanotechnology [95-97], in the following paragraphs some examples of applications in the fields of plasmonics, sub-diffractive optics, solar energy harvesting and optical sensing and biosensing are reported.

Metallized diatom valves as efficient SERS substrates Since diatom frustules are regularly nanopatterned dielectric structures, they are susceptible, if properly metallized, to trigger plasmonic effects. One of the main application of plasmonics in sensing is given by the already mentioned SERS, where localized surface plasmons (LSPs) supported by metallic nanoparticles or periodic metallic nanostructures are exploited to boost the Raman scattering signal coming from molecules in their proximity, allowing the detection of low concentrations (down to single molecules) of analytes. First attempts to use diatom frustules as SERS substrates initially involved the fabrication of silver replicas of Synedra sp. and Thalassiosira sp. valves by chemical removal of silica after silver evaporation [98], allowing reaching an enhancement factor (EF) of about $\simeq 10^{6}$ using rhodamine as testing analyte. EF is defined as:

$$
E F=\frac{I_{S E R S} / N_{\text {surf }}}{I_{R S} / N_{v o l}},
$$

where $I_{S E R S}$ and $I_{R S}$ stand for SERS and Raman intensities, $N_{\text {surf }}$ is the average number of molecules adsorbed onto the metallic substrate in the scattering area for the SERS measurements, and $N_{v o l}$ is the number of molecules in the scattering volume for spontaneous Raman scattering measurements. 
Photonic-plasmonic hybrids have been obtained by chemical attachment of metallic nanoparticles to diatom frustules, enabling the efficient coupling of the valve guidedmode resonances with the LSPs supported by the nanoparticles, thus ensuring high quality factors of the local electric field. Ren et al. [99] obtained silver nanoparticles self-assembly to amine-functionalized Pinnularia sp. valves and tested them as SERS substrates, obtaining an increase in EF up to 12 times comparing the acquired spectra from rhodamine $6 \mathrm{G}$ dropped onto the hybridized valves with respect to a planar glass slide which had undergone the same metallization process. This kind of substrate has been used also in a SERS-based sandwich immunoassay system for the detection of antibody-antigen recogniton [100], allowing a detection limit for mouse IgG down to $10 \mathrm{pg} / \mathrm{mL}$. Finally, monolayers of Pinnularia sp. frustules coated with silver nanoparticles have also been succesfully used in experiments based on the combination of SERS with ultrathin layer chromatography (UTLC) [101].

Direct thermal evaporation of gold onto Pseudonitzchia multistriata valves with no silica removal has been applied by Managò et al. [38] for the realization of SERS substrates aimed at the detection of the biochemical composition of the membrane of red blood and B-leukemia REH cells. The peculiar, hierarchical ultrastructure of $P$. multistriata frustules, where several interacting periodicities can be identified, guarantees an extremely efficient coupling with external optical radiation. Furthermore, the presence of an extruded lateral edge on one side of the valve allows interacting with the cell under analysis avoiding steric hindrance, thus ensuring the acquisition of membrane Raman signals with no interference from other cellular compartments. The EF of these substrates has been quantified starting from the spectra of a biphenyl4-thiol (BPT) monolayer self-assembled onto a metallized valve and equals, for a 40 nm thick gold film, $(4.6 \pm 0.9) \times 10^{6}$.

Besides the enhancement of Raman signals, other plasmonic effects induced by metallized diatom frustules have been obesrved. For example free-standing, gold replicas of Coscinodiscus asteromphalus valves, obtained by an amine-amplifying surface functionalization followed by electroless gold deposition and selective removal of silica by dissolution in a HF solution, show transmission maxima at infrared wavelengths not found in the starting silica valves nor in flat non-porous gold films used as reference [102]. This extraordinary optical transmision (EOT) effect has been ascribed to the excitation of surface plasmons by scattering from individual holes and consequent interference with incoming light. Surface plasmon-mediated EOT can find applications in integrated optics and in chemical and biological sensing [103,104]. Sub-diffraction light squeezing assisted by Optical Eigenmodes technique The ability of centric diatom valves to focus and confine incoming light in a tiny spot can be improved by the combination with sub-diffraction, far-field techniques, achieving what can be defined as a "bio-assisted" super-resolution. In particular this has been accomplished by the application of optical eigenmodes (OEi) technique to single valves of Arachnoidiscus sp. diatoms [105]. OEi technique [106-108] is a structured illumination approach based on the use of $N$ probe fields to construct the finite intensity $\mathbf{M}^{(\mathbf{0})}$ and second order momentum $\mathbf{M}^{(\mathbf{2})}$ operators for a given region of interest (ROI). The eigenmode with the smallest eigenvalue of $\mathbf{M}^{(2)}$ represented in the normalized eigenmode base of $\mathbf{M}^{(\mathbf{0})}$ corresponds to the smallest achievable spot for the given ROI and probe fields. Experimentally the probe fields are obtained by using a spatial 
light modulator (SLM). In the case under consideration, a single Arachnoidiscus sp. valve is illuminated by a focused laser beam $(\lambda=532 \mathrm{~nm})$ modulated by a SLM used to probe and detect the OEi of the system. The combination of probe beams which minimizes the second order momentum of the spot transmitted by the valve is recorded. The valve alone is able to confine light at a distance of about $30 \mu \mathrm{m}$ along the optical axis, while, when illuminated by OEi fields, sub-diffractive focal spots take place at different distances along the illumination path which are even smaller than the ones obtainable with OEi in absence of the valve. For circular polarization and at a distance of $20 \mu \mathrm{m}$ from the valve plane, the focal spot full width at half maximum (FWHM) reaches a value of $0.21 \lambda / \mathrm{NA}$ (with NA numerical aperture and $0.51 \lambda / \mathrm{NA}$ classical resolution limit), surpassing the performance of other sub-diffraction far field techniques such as OEi pupil filters [106-109], random lenses based on material with high refractive index [110] or specially engineered and micro-fabricated binary mask super-lenses [111,112]. At the origin of this efficient sub-diffraction confinement of radiation stands the interplay between the OEi capability to probe the optical degrees of freedom (ODOF) of the system and the valve nanostructure enabling an efficient access to those ODOF.

Enhancement of efficiency in dye-sensitized solar cells In a dye-sensitized solar cell (DSSC), solar radiation is absorbed by a photosensitive dye, usually a ruthenium complex, bound to a photoanode enriched with a thin film of $\mathrm{TiO}_{2}$ nanoparticles. In its photoexcited state, the dye injects electrons in the conduction band of the semiconducting $\mathrm{TiO}_{2}$. Finally, the electrons diffuse through titania to the working electrode while the dye is regenerated by a liquid electrolyte [113-115]. In 2013 Toster et al. [116] used titania-enriched diatom frustules as photoanodes in a DSSC. Frustule coating with titania nanoparticles was performed by means of plasma treatment, avoiding the chemical modification of biosilica surface by functional groups. After only three cycles of treatment, an increase in conversion efficiency of about $30 \%$ with respect to conventional DSSCs has been observed. The effect is ascribed to the high effective surface area which characterizes the frustule-titania hybrids and increases the density of dye molecules. Furthermore, the multiple scattering events and light trapping induced by frustules imply an enhanced interaction between incoming photons and dye electrons. A further increase in cell efficiency (up to 35\%) and stability has been recently obtained by Bandara et al. [117] by substituting the liquid electrolyte with a gel polymer electrolyte and avoiding the application of volatile solvents for its preparation. In this case the titania-frustules hybrids have been obtained by spin coating of a mixture prepared using $\mathrm{TiO}_{2} \mathrm{P} 90$ powder and diatom biosilica.

Besides the use of modified diatom frustules to enhance the efficiency of a DSSC, a biomimetic approach in the design of the active layer of a generic solar cell can be employed. Several periodic light trapping nanostructures have been proposed through years for silicon-based solar cells, such as photonic crystals [118,119], triangular or pyramid gratings [120,121], and plasmonic nanostructures [122,123]. All these solutions are based on an ad-hoc, intuition-based methodology aimed at the predefinition of the topology of the light-trapping structure and often do not allow to reach the optimal design. Inspired by the unique hierarchical periodic structures of diatom frustules modeled through milions of years of evolution, Wang et al. [124] applied genetic algorithms based on nongradient topology optimization in combination with rigor- 
ous coupled wave analysis (RCWA) method to design highly efficient light trapping structures characterized by an enhancement factor over three times the Yablonovitch limit.

Optical sensing and bio-sensing Nanostructured diatom biosilica is characterized by visible radiation emission after UV excitation [93]. This process, typical of several forms of nanostructured silica such as silica nanoparticles, oxidized porous silicon, sol gels, silicon-oxide thin films, and silica-based mesoporous materials, is to be ascribed mainly to a variety of surface defects, including non-bridging oxygen hole centers, neutral oxygen vacancies, silanol groups, and to the recombination of selftrapped excitons localized by self-induced lattice distortions in presence of strong electron-phonon interactions [125-127]. Furthermore, in the case of diatoms, also organic residuals incorporated in the frustule silica matrix contribute to visible photoluminescence [72,128]. De Stefano et al. [129] firstly observed that green emission from Thalassiosira rotula frustules is sensitive to the chemical composition of the environment, both in terms of emission wavelength and peak intensity. In particular, in presence of growing concentrations of electrophilic gases or vapors such as $\mathrm{NO}_{2}$, acetone, and ethanol, the photoluminescence spectra are progressively quenched (due to attraction of electrons from silica skeleton) and red-shifted (due to adsorption of the volatile substances into the porous matrix of the frustule). On the other side, the exposure to nucleophilic gases like xylene and pyridine causes, apart from the above-mentioned red shift in the emission spectra, a noticeable enhancement of the photoluminescence intensity following electron donation to non-radiative defects of the frustule. This effect can be exploited as transducing mechanism in gas optical sensing and has been studied also for other species (C. wailesii [130] and Cocconeis scutellum [131]), which allowed understanding the effect of frustule morphology on the response of its photoluminescence and on the sensitivity of the process.

The ability to chemically modify the frustule surface in order to covalently link bioprobes such as enzymes, antibodies, or single nucleic acid strands paved the way to the realization of label-free, diatom-based optical biosensors. An example is given in Ref. [132], where Cyclotella sp. frustules have been functionalized by reaction with 3-aminopropyltrimethoxysilane (APS), bissulfosuccinimidyl suberate (BS3) as crosslinker, and rabbit IgG antibody as anchored bioprobe. The characteristic blue photoluminescence emitted by the frustules after excitation at $337 \mathrm{~nm}$ was amplified by a factor of six after IgG binding and by a further factor of three after immunocomplex formation with the complimentary antigen anti-rabbit $\mathrm{IgG}$, while no response is observed in presence of a non-complimentary antigen. The immunocomplex binding followed a Langmuir adsorption isotherm with a binding constant of $2.8 \pm 0.7 \times 10^{-7} \mathrm{M}$.

\section{Artificial photonic structures: metasurfaces}

Metamaterials are artificial structured materials, not existing in nature, endowed with unique physical properties. Their fabrication is complex, in many cases making them of impractical application. Metasurfaces (MSs), the two-dimensional counterpart of metamaterials, are characterized by thicknesses of the order of few tens of nanometers when designed for optical applications, viz. about one order of magnitude lower than 
the wavelengths of the electromagnetic (EM) fields they interact with. Unlike threedimensional metamaterials, MSs can be easily fabricated exploiting well established micro- and nano-electronic techniques. These planar and compact structures open the way to the miniaturization and integration of optical devices that still rely on basic and bulky configurations (such as lenses, prisms, filters, switches, etc.), increasing their performance, and to the design of new photonic components with unexpected functionalities in terms of optical field localization and manipulation. MSs are usually composed of nanostructures ordered in arrays suitably engineered, that experience resonant interaction with the incoming EM radiation. The dimension of these nanostructures (also called nanoantennas, NAs), as well as their spacing, are lower than the wavelength of the incoming light. Simply adjusting their shape and size, as well as the array periodicity, it is possible to control and manipulate light below the diffraction limit, down to the nanoscale.

First proposals of devices beating the diffraction limit date back to the 40s [133], with the study of diffraction of visible light through a subwavelength hole in an infinite thin metal film. After several years, the discoveries of extraordinary optical transmission [134] and perfect imaging [135] properties, based on plasmonic effects in two dimensional structured metals, paved the way to the realization of new nanophotonic MSs with extraordinary response [136-140].

MSs have been used to manipulate polarization [141,142], phase [136,143-146], and amplitude of EM fields [147,148]. More sophisticated MSs, transforming both phase and polarization, have been recently realized to produce beams possessing angular orbital momentum (OAM) [149] or vortex waves [150-152], holograms [153,154], and stable beam traction [155]. In order to enable these extraordinary responses, it is imperative to strongly enhance the interaction between light and matter. A rich diversity of MSs, and their applications, has been reported so far. They can be grouped as dielectric $[156,157]$ and plasmonic $[158,159]$. Moreover, in addition to optical waves, similar concepts have been applied to microwave [160], non-hermitian [161], and also acoustic waves [162].

MS design requires a solid model that describes its physical structure (i.e., material, geometry and size of the scattering structures, substrate parameters, layer configuration and thickness) and provides insight into its physical behavior. They are best described, according to Huygens principle, as "surface polarization current sheets" via continuous (locally homogeneous) bianisotropic surface susceptibility tensorial functions; inserting the corresponding surface polarization densities into Maxwell equations results in electromagnetic sheet transition conditions, which consist in the key equations to solve in the design of metasurfaces [148,163-165].

Critical issues are involved in the correct design of MSs, such as: high optical losses often associated with the resonant structures, large-scale CMOS-compatible nanofabrication techniques and potential incorporation of active control elements. Furthermore, practical MS devices could require robust operation in high-temperature environments, caustic chemicals, and intense EM fields. Novel material platforms (refractory plasmonic materials, epitaxial noble metal, silicon, graphene, phase change materials, and metal oxide) that offer resilient, low-loss and tunable metasurface designs are driving new and promising routes for overcoming these obstacles. 


\section{Plasmonic metasurfaces}

For centuries, metals were employed in optical applications only as mirrors and gratings. New vistas opened up in the late 1970s and early 1980s with the discovery of surface-enhanced Raman scattering and the use of surface plasmon (SP) resonances for sensing. However, it was not until the 1990s, with the appearance of accurate and reliable nanofabrication techniques, that plasmonics blossomed.

A plasmon is a quantum of free electron oscillation whose excitation is accompanied by a dramatic localization and enhancement of the electric field associated with the interacting light. These characteristics, combined to the ability of modifying the local density of photonic states and to the ultra-fast response to surrounding stimuli, are central to a variety of novel techniques in nanoelectronics, sensing and imaging, finding extensive applications in different fields, from bio-medicine and environmental monitoring, to telecommunications and photovoltaics. The properties of SPs are strongly related to materials and structures, so that metals, semiconductors and two-dimensional materials with various morphologies can have plasmonic resonances ranging from ultraviolet to far infrared.

Surface plasmon polaritons (SPPs) are evanescent waves excited at the interface between a dielectric and a conductor due to the coupling of the EM fields to oscillations of the conductor's electron plasma. Their physical properties can be investigated by solving the Maxwell's equations at the interface between a semi-infinite conductor and a semi-infinite dielectric, each characterized by a complex permittivity, respectively given by

$$
\begin{aligned}
& \epsilon_{c}=\epsilon_{c}^{\prime}+i \epsilon_{c}^{\prime \prime} \\
& \epsilon_{d}=\epsilon_{d}^{\prime}+i \epsilon_{d}^{\prime \prime}
\end{aligned}
$$

where $\epsilon^{\prime}$ and $\epsilon^{\prime \prime}$ are the real and imaginary parts of $\epsilon$. Referring to Fig. 9, for waves confined at the interface (i.e with evanescent decay in z-direction) and propagating along x-direction, only surface modes for TM polarization can exist (viz., only $E_{x}, E_{z}$ and $H_{y}$ are non-zero). The dispersion relation defining the propagation constant $\beta$ of a SPP at the interface between the two half spaces is given by $[7,166]$ :

$$
\beta=k_{0} \sqrt{\frac{\epsilon_{c} \epsilon_{d}}{\epsilon_{c}+\epsilon_{d}}}
$$

where $k_{0}=\omega / c=2 \pi / \lambda$ is the wave vector of the propagating wave in vacuum. This expression is valid for both real and imaginary part. For lossless (undamped) materials $\left(\epsilon_{c}^{\prime \prime}, \epsilon_{d}^{\prime \prime}=0\right)$, SPPs are demonstrated to only exist if the permittivities $\epsilon_{c}^{\prime}$ and $\epsilon_{d}^{\prime}$ are of opposite signs and $\epsilon_{c}^{\prime}<\epsilon_{d}^{\prime}$. As the permittivity of dielectric materials is usually positive, the adjacent conducting material has to show a real negative dielectric function $\epsilon_{c}^{\prime}<0$ (this condition is fulfilled for metals at frequencies below the bulk plasmon frequency $\omega_{p}, v i z$, the plasma frequency of the free electron gas).

Metals such as gold, silver, and aluminum exhibit a negative real part of permittivity in the visible and near infrared regions of the electromagnetic spectrum. Due to its bound nature, the excitation of a SPP can only take place if there is a match 
Fig. 9 Schematic representation of the propagation geometry for a SPP. The wave propagates along the $\mathrm{x}$-direction

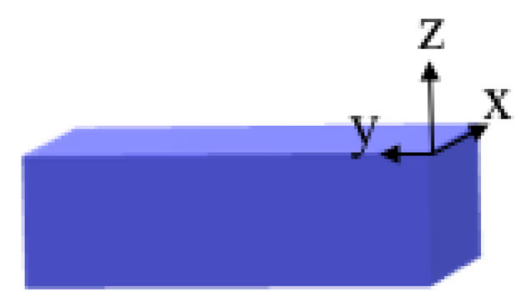

between its momentum and that of the incoming light beam. It is easy to demonstrate that, for a given frequency, a free-space photon has always less momentum than a plasmon because of their different dispersion relations [167]. Coupling can only be achieved using a suitable medium to match the photon and surface plasmon wave vectors and momenta. Thus, special phase-matching techniques such as grating [168] or prism coupling $[169,170]$ are required for their excitation via three-dimensional beams. Radiation into the metal occurs in the transparency regime $\omega<\omega_{p}$. Between the regime of the bound and radiative modes, a frequency gap region with purely imaginary $\beta$ prohibiting propagation exists.

In the process of optical excitation of surface plasmons, a portion of the energy of the light wave is transferred into the energy of a surface plasmon and dissipated in the metal film, which results in a drop of intensity of the light wave. In addition to the change in the intensity, the light wave exciting a surface plasmon undergoes a change in phase [171].

Since conduction electron excitation suffers both from free-electron and interband damping, a considerable imaginary part of the permittivity still exists, which causes the propagation constant of a surface plasmon to have a non-zero imaginary part. The imaginary part of the propagation constant is associated with the damping of SPPs with an energy attenuation length called propagation length $L=(2 \Im(\beta))^{-1}$. The real part of the propagation constant is related to the effective index through the relation: $n_{\text {eff }}=\Re(\beta) / k_{0}$.

Due to the damping, the wave vector of the bound SPPs approaches a finite limit at the surface plasmon frequency $\omega_{s p}$. This limitation puts a lower bound both on the wavelength $\lambda$ of the surface plasmon and also on the amount of mode confinement perpendicular to the interface. The quasi-bound, leaky part of the dispersion relation between $\omega_{s p}$ and $\omega_{p}$, is allowed, in contrast to the case of an ideal conductor, where $\Re(\beta)=0$ in this regime.

SPPs at frequencies close to $\omega_{s p}$ exhibit large field confinement at the interface and a subsequent small propagation distance due to increased damping. The better the confinement, the lower the propagation length. This characteristic trade-off between localization and loss is typical for plasmonics. We note that field confinement below the diffraction limit of half the wavelength in the dielectric can be achieved close to $\omega_{s p}$. In the metal itself, the fields fall off over distances on the order of $20 \mathrm{~nm}$ over a wide frequency range spanning from the visible to the infrared.

Localized plasma oscillations can be excited in metallic subwavelength structures or nanoparticles, where the electric field of incoming radiation can polarize the conduction electrons. The resulting free electron oscillations are distributed over the 
Fig. 10 Sketch of a

homogeneous sphere placed into

an electrostatic field

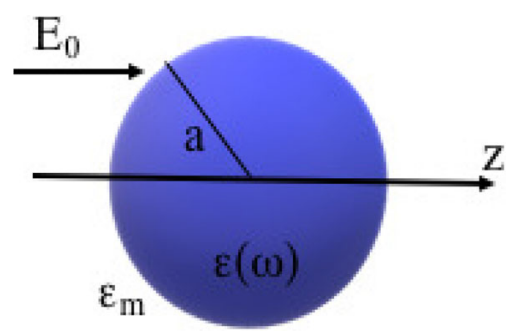

nanoparticle volume and are localized within the particle. These plasmon oscillations are called localized surface plasmons (LSPs). The displacement of the electron clouds from the lattice generates a restoring force that tries to pull the electrons back into the lattice. The nanoparticle therefore acts as an oscillator driven by the incoming field together with restoring Coulomb force and behaves as a simple dipole in the direction of the electric field. When the frequency matches the resonance-frequency defined by the shape of the particle, a LSP resonance (LSPR) occurs, enhancing local field amplitude. The nanostructures can also introduce a local phase shift to the incoming light beam and manipulate its wavefront.

A theoretical treatment of localized surface plasmons is lengthy and beyond the scope of this review. We refer the interested reader to [7]. A simplified treatment describing the phenomenon in a macroscopic way derives from the scattering theory applied to a small, sub-wavelength conductive nanoparticle in an oscillating EM field. The curved surface of the particle exerts an effective restoring force on the driven electrons, so that a resonance can arise, leading to field amplification both inside and in the near-field zone outside the particle. Another consequence of the curved surface is that plasmon resonances can be excited by direct light illumination, in contrast to propagating SPs, where the phase-matching condition has to be satisfied. The interaction of a particle of size $d$ with the EM field can be analyzed using the simple quasi-static approximation provided that the particle dimension is much smaller than the wavelength of light in the surrounding medium. For analytical treatment, a convenient geometry is represented by a homogeneous, isotropic sphere of radius $a$ located at the origin in a uniform, static electric field $\mathbf{E}=E_{0} \mathbf{z}$ (see Fig. 10).

In this case, the phase of the harmonically oscillating electromagnetic field is practically constant over the particle volume, so that one can calculate the spatial field distribution by assuming the simplified problem of a particle in an electrostatic field. Assuming that the surrounding medium is isotropic and non-absorbing with dielectric constant $\epsilon_{d}$, and the dielectric response of the sphere is further described by the dielectric function $\epsilon_{c}(\omega)$, the applied field induces a dipole moment inside the sphere of magnitude proportional to $|\mathbf{E}|$. Introducing the polarizability $\alpha$ as function of the dipole moment $p$ defined as:

$$
p=\epsilon_{0} \epsilon_{d} \alpha E_{0}
$$


one obtains the (complex) polarizability of a small sphere of sub-wavelength diameter in the electrostatic approximation:

$$
\alpha=4 \pi a^{3} \frac{\epsilon_{c}-\epsilon_{d}}{\epsilon_{c}+2 \epsilon_{d}} .
$$

It is apparent that the polarizability experiences a resonant enhancement when $\left|\epsilon_{c}+2 \epsilon_{d}\right|$ is a minimum, which for the case of small or slowly-varying $\Im\left(\epsilon_{c}\right)$ around the resonance simplifies to the Fröhlich condition:

$$
\Re\left(\epsilon_{c}(\omega)\right)=-2 \epsilon_{d} .
$$

This condition expresses the strong dependence of the resonance frequency on the dielectric environment: the resonance red-shifts as $\epsilon_{d}$ is increased. Metal nanoparticles are thus ideal platforms for optical sensing of changes in refractive index. As expected, the resonance in $\alpha$ also implies a resonant enhancement of both the internal and dipolar fields. It is this field-enhancement at the plasmon resonance on which many of the prominent applications of metal nanoparticles in optical devices and sensors rely. From the viewpoint of optics, it is interesting to note that another consequence of the resonantly enhanced polarization is a concomitant enhancement in the efficiency with which a metal nanoparticle scatters and absorbs light. The theory of scattering and absorption of radiation by a small sphere predicts a resonant field enhancement due to a resonance of the polarizability $\alpha$ if the Fröhlich condition is satisfied. Under these circumstances, the nanoparticle acts as an electric dipole, resonantly absorbing and scattering electromagnetic fields. This theory of the dipole particle plasmon resonance is strictly valid only for vanishingly small particles. The relations outlined above provide a reasonably good approximation for spherical or ellipsoidal particles with dimensions below $100 \mathrm{~nm}$ illuminated with visible or near-infrared radiation. However, for particles of larger dimensions, where the quasi-static approximation is not justified due to significant phase-changes of the driving field over the particle volume, a rigorous electrodynamic and numerical approach is required [172,173].

The localized plasmon resonance frequency of a single metallic nanoparticle depends on the size, shape, composition, and local optical environment of the particle [173], and typically occurs in the visible and near-infrared part of the spectrum for nanostructures made of noble metals $(\mathrm{Au}, \mathrm{Ag}, \mathrm{Cu})$.

However, localized surface plasmons are generally of greater spectral width when compared to propagating surface plasmons: as an example, the resonance full width at half maximum (FWHM) is typically more than $100 \mathrm{~nm}$ for LSPs of Au nanostructures, compared to a spectral width of about $50 \mathrm{~nm}$ for propagating SPs. The quality factor of the resonance, $Q$, which in an oscillator is intrinsically expressed as the ratio of energy stored to the energy lost by an oscillator, can be estimated as $Q=\lambda_{\text {res }} / \Delta \lambda$ ( $\lambda_{\text {res }}$ is the resonance wavelength, and $\Delta \lambda$ is the full width at half maximum of the resonance) and is large when $\Im\left[\epsilon_{c}(\omega)+2 \epsilon_{d}(\omega)\right]$ is small. For metals, the dielectric constant is a complex value so that it is not possible to have a zero value for the denominator in Eq. 7 and $Q$ factors of $\simeq 10-20$ are found to be typical for most LSP resonances. An improvement in the quality factor of LSPs was originally expected 
from appropriate engineering of the shape and size of nanostructures. However, studies involving various geometries, including nanotriangles [174,175], nanorods [176], nanostars $[177,178]$, and nanocrosses $[179,180]$ did not lead to remarkable LSP resonance narrowing. Actually, in the quasi-static approximation [181] the quality factor of the plasmon resonance depends only on the dielectric function of the metal at the plasmon frequency, once material and plasmon frequency have been fixed. Thus, while the shape and environment do influence the LSP frequency, the quality factor is independent on the nanostructure shape and on the dielectric surrounding medium. Moreover, beyond the quasi static regime the plasmon resonance is damped by two competing processes which contribute to broaden the LSP resonance: a radiative decay process into photons, and a non-radiative decay due to absorption. The radiative decay process into photons is dominating for larger particles; the non-radiative decay is due to absorption (creation of electron-hole pairs via either intraband excitations within the conduction band or interband transition from lower d-bands to sp-conduction-band for noble metal nanoparticles). These two damping processes can be incorporated into a simple two-level model of the plasmon resonance [182].

\section{Array of nanoantennas: field enhancement and loss mechanisms}

Fortunately, the limitations on the $Q$ factor of LSPs associated with individual nanostructures discussed above can be largely overcome when nanostructures are arranged in arrays. The electromagnetic fields related to the LSP mode of one nanoparticle may then act to influence the response of neighboring nanoparticles. Additional shifts of the plasmon resonance are expected to occur in nanoparticle arrays due to EM interactions between the localized modes. For small particles, these interactions are essentially of a dipolar nature, and the particle ensemble can be treated, in a first approximation, as an ensemble of interacting dipoles. Assume that the particles of size $a$ are arranged within one or two-dimensional ordered arrays with inter-particle spacing $d$. Assuming $a \ll d$, so that the dipolar approximation is justified, and the particles can be treated as point dipoles, two regimes have to be distinguished, depending on the magnitude of the inter-particle distance $d$ : near-field and far-field coupling.

In the near-field coupling regime, closely spaced particles characterized by $d \ll \lambda$ interact with a distance dependence through a dominating factor $d^{-3}$, and the particle array can be described as an array of point dipoles interacting via their near-field. In this case, strong field localization in nano-sized gaps between adjacent particles can be observed [183]. So, scattering is drastically suppressed for closely spaced particles, and the fields are instead highly localized at interstitial sites. Similar inter particle junctions, therefore, serve as hot-spots for field enhancement. These interactions lead to significant spectral shifts of the plasmonic resonances and a modification and splitting of their line-shapes due to the hybridization of the plasmonic modes [184-186]. In particular, such hybridization can lead to the generation of antisymmetric modes [187], which provide slightly narrower resonances (characterized by a FWHM of about 50 $\mathrm{nm}$ ). However, these modes are typically dark and cannot easily be excited by incident light. 
In the far-field coupling regime, largely spaced particles interact with far-field dipolar coupling (i.e., via their scattered radiation fields) with a distance dependence through a dominating factor $d^{-1}$. Far-field coupling has pronounced influences on the plasmon line shape, both in terms of resonance frequency and of spectral width. When a number of particles are randomly distributed, the scattered fields impinging on a given particle have no particular phase relationship, and the effects of the scattered fields are relatively minor [188]. However, when metal nanoparticles (nanoantennas) are arranged in a periodic array, and the period is comparable to the wavelength of the incident light, then under appropriate conditions the scattered fields impinging on a given particle can arrive in phase with the incident light. The scattered fields correspond to diffraction of the incident light in the plane of the array. By using the right combination of nanoparticle size and shape, together with an appropriate array period and symmetry, the light scattered by each nanoparticle can be put in phase with the plasmon resonance induced in its neighbor by the incident light, thereby reinforcing the resonance in the neighboring particles. Thus, the quality factor of the resonance can significantly increase by appropriate tuning of the array. When extended over a large array of nanoparticles [189], such plasmonic surface lattice resonances (diffractively coupled localized surface plasmon resonances) can lead to a remarkable narrowing of the resonance width (down to a few $\mathrm{nm}$ ), as well as to related phenomena such as a dramatic enhancement of both absorption and the local electric fields near the nanostructures. However, observation of diffractively LSP resonances requires the incident light to be spatially coherent over a large area (i.e., covering a sufficient number of particles) to ensure useful interference of the light scattered by the arrayed nanoparticles. Moreover, the interactions between metal nanoparticles can be further enhanced by providing additional coupling pathways, for example in the form of propagating SPs for particle arrays fabricated on a conductive substrate [190], or benefit from a media exhibiting optical gain [191].

LSPs have emerged as an attractive alternative to propagating SPs in a wide range of applications, primarily because plasmons can be excited without fulfilling momentum matching conditions. Moreover, they benefit from standard bottom-up fabrication routes for metal nanoparticles, as well as from top-down nanolithography techniques for array definition. A limit can be the lack of post fabrication dynamic control of plasmonic resonance. The resonant wavelength of the NAs is dependent on their geometrical parameters and spatial arrangement. Recently, graphene with its exceptional opto-electrical properties represented a good platform to electrically modulate plasmonic resonances [192].

LSP-based metasurfaces have been showing a series of unique properties and functionalities which find application in sub-diffraction lensing [193], spectroscopy [194], monochromatic [19] and color holography [195], polarization conversion [196-198], vortex plates [199], invisibility and cloaking [200], polarization-selection [201], photovoltaics [122], and super-sensitive nanosensing [202-205].

Despite all these promising applications, undesired optical losses and related heat generation in metals severely limit their use in replacing conventional optical elements. Neither in the best plasmonic materials, with small imaginary part and large negative real part of the permittivity, the losses can be avoided entirely. Even for an ideal plasmonic material with negligible loss, nanostructuring the metal causes the magnetic 
field of an incoming EM wave to be truncated while interacting with the free electrons of the structure. When the size of nanostructure is roughly sub-wavelength $(a<\lambda / 2 n$, where $n$ is the refractive index), the field spatial dependence changes and the magnetic field is truncated. Therefore the magnetic energy is much smaller than the electric energy. This makes self-sustaining oscillations impossible without the conversion of stored magnetic energy into kinetic energy of electrons, inducing a loss mechanism termed Landau damping [206].

Absorption process requires a phonon or imperfection, and produces two hot carriers. The absorption probability due to phonons and defects is proportional to $\gamma_{p h}(\omega) \omega_{p}^{2} / \omega^{2}$, and is weakly dependent on frequency (where the scattering probability with phonons is typically $\left.\gamma_{p h}(\omega) \simeq 10^{14} \mathrm{~s}^{-1}\right)$. It is high due to high density of states above the Fermi level available for the transition. Another absorption process is due to electron-electron scattering, that ends up with four carriers having less energy; this process is strongly frequency dependent, and at visible range is comparable with the electron-phonon scattering. Another mechanism that gives absorption is the cited Landau damping [206], in which direct absorption is assisted by the propagating SP momentum, and that, for particle with $a<10 \mathrm{~nm}$, shows rates comparable with the other ones. Finally, in metals there are always transitions between bands, without generation of hot carriers. In fact, only the scattering with phonons and the Landau damping produce hot carriers that thermalize in few tens of femtoseconds.

Compared to dielectric metasurfaces (which will be discussed in section 8), plasmonic MSs (PMSs) are therefore inherently less efficient, and a tradeoff between field confinement and loss must be found in practical applications, that anyway can take advantage of tight field confinement, broad bandwidth, and small device footprint. Of course, PMSs are particularly useful when optical losses are desired, such as in heating or absorbing device systems.

To summarize, in PMSs the diffraction limit can be exceeded with the cooperation of free carriers, but the energy gets stored in the form of kinetic energy. Hence, it is lost at the rate of electron's scattering in metals, which is on the scale of tens of femtoseconds. The density of photon states in PMSs is much higher than in free space, and the rate of spontaneous emission can be increased many times. The emission involves the states of the motion of free electrons that couple into the original photon states. So, correlating the losses to the Purcell effect, the field enhancement in plasmonic MSs is obtained by coupling energy into lossy electronic states, and so the energy has a higher probability to be dissipated rather than radiated into free space. That is why PMSs work well for originally weak and inefficient optical processes, and have been assuming increasing interest in sensing, where there is no need for high absolute efficiency, in Raman and IR spectroscopies, as well as in several applications requiring thermal management. In the next section, we focus our attention on PMSs used as vehicle to strongly enhance signal detection in Raman and IR spectroscopy. 


\section{Sensing application: surface enhancement spectroscopy}

The high field enhancement offered by PMSs is of fundamental importance especially in those physical systems where the interaction between light and matter is intrinsically weak. In particular, besides sensing exploiting the natural dependence of plasmonic resonances on the changes of the surrounding medium [207], one of the application field of PMSs which is gaining high interest are the Raman and infrared (IR) spectroscopies, based on the interaction of light with the molecular vibrations of the system under analysis, capable of providing both quantitative and qualitative information about the target analyte [208].

Raman and mid-IR spectroscopy are complementary techniques, and usually both are required to completely measure the vibrational modes of a molecule. They differ in the way the photon energy is transferred to the analyzed molecule by changing its vibrational state. In the case of mid-IR spectroscopy, interaction between light and matter is based on a resonance condition involving the transition between vibrational energy levels mediated by the electric dipole. In the case of Raman spectroscopy, the interaction between light and matter depends on an off-resonance condition involving the polarizability of the molecule. Here, the incident photon has much more energy than the vibrational quantum one, and transfers part of it to the molecular vibration while the remaining is scattered as a photon with reduced frequency (two-photon inelastic light scattering event) [208]. In general, symmetric vibrations of nonpolar groups give rise to better Raman spectra, while IR spectroscopy is more sensitive to the asymmetric vibrations of polar groups. The IR and Raman vibrational bands are characterized by their frequency (energy), intensity (polar character or polarizability), and band shape (environment of bonds). Diatomic molecules such as $\mathrm{H}_{2}, \mathrm{~N}_{2}$, and $\mathrm{O}_{2}$ have no dipole moment and are IR inactive (but Raman active), while heteronuclear diatomic molecules such as $\mathrm{HCl}, \mathrm{NO}$, and $\mathrm{CO}$ do have dipole moments and have IR active vibrations. Since the vibrational energy levels are unique to each molecule, IR and Raman spectra provide a "fingerprint" of a particular molecule. The frequencies of these molecular vibrations depend on the masses of the atoms, their geometric arrangement, and the strength of their chemical bonds. The spectra provide information on molecular structure, dynamics, and environment. However, both IR and Raman spectroscopies are characterized by extremely low molecular absorption/scattering cross-sections of the order of $10^{-20}$ and $10^{-30} \mathrm{~cm}^{2}$, respectively. Therefore, a large amount of material or of photon energy, as well as a long time of acquisition, are required for accurate measurements. This limit can be overcome in surface enhanced Raman spectroscopy (SERS) and in surface enhanced infrared absorption (SEIRA) spectroscopy techniques thanks to the field enhancement offered by PMSs. Vibrational modes of molecules located at the surface of PMSs, where the EM fields are strongly confined, are enhanced by several orders of magnitude enabling a spectroscopic characterization with unprecedented sensitivity [209,210]. The detection of molecules at extreme low concentrations, together with the capability to perform label-free and non-invasive analysis, has been promoting the use of both SERS and SEIRA techniques not only in medicine and pharmacology, but also in emerging fields such as trace chemical detection in environmental analysis, homeland security, food safety and forensic sciences. 
In many aspects, the most fundamental metric for SERS and SEIRA is the enhancement factor (EF), which quantifies the amplification of the signal for a specific molecular vibrational mode. SERS and SEIRA experiments require that both incident radiation at definite frequency $\omega_{i n c}$ and the molecular vibrational mode (at the eigenfrequency $\omega_{v i b}$ equal or very close to $\omega_{i n c}$ ) are in resonance with the LSPR of the metal MSs. In particular, the EF in SERS is defined as [211]:

$$
E F_{S E R S}=\frac{\left|E_{L O C}\left(\omega_{i n c}\right)\right|^{4}}{\left|E_{i n c}\right|^{4}}
$$

where $\left|E_{L O C}\left(\omega_{i n c}\right)\right|^{4}$ is given by the product of the squared local field due to the nanoantenna at $\omega_{i n c}$ times the squared "outgoing" Raman scattered field (assuming $\left.\omega_{i n c} \simeq \omega_{i n c}-\omega_{v i b}\right)$ and $E_{i n c}$ is the incident field at $\omega_{i n c}$. Therefore, an increase of local field due to the MS, i.e., $E_{l o c} / E_{i n c}=10^{2}$, for example, results in an overall increase of the SERS intensity by a factor of $10^{8}\left(\left(E_{l o c} / E_{i n c}\right)^{4}\right)$. It is worth noting that, since the electric field strength of dipolar radiation scales with the distance as $r^{-3}$, a SERS distance dependence as $r^{-12}$ is expected, which means that SERS is a surface-selective effect. Very high EFs are only observed in highly localized regions, called "hot spots", in the junctions between two nanostructures. Thus, the ability to precisely control the surface characteristics is critical to fabricate reliable and robust SERS substrates.

Since the enhancement process, as described in Eq. 9, involves only the MS's nanostructures and the incident photon, it does not require the presence of the molecules but only the frequency conversion from $\omega_{i n c}$ to $\omega_{i n c}-\omega_{v i b}$. The experimental determination of EF requires measurements of the SERS intensity for the adsorbed molecule on the MS, relative to the normal Raman intensity of the same, "free" molecule in solution. The two intensities must be normalized to the corresponding number of molecules on the surface and in solution, respectively.

Similarly, the enhancement mechanism in resonant SEIRA is due to the interaction between plasmonic and molecular excitations, which require a model accounting for resonant coupling, but a detailed understanding of this mechanism does not exist yet. To fit experimental SEIRA spectra, modified functional forms of Fano resonances [212] or coupled harmonic oscillators [213] are used. In SEIRA experiments the plasmonic mode is strongly driven by incident infrared field; in contrast, the incident field only weakly interacts with the molecular vibrational mode which is excited by the coupling to the nanoantenna's plasmonic mode. Consequently, the vibration is not directly observed as an absorption feature in the spectrum, but appears as a modulation on top of the plasmonic resonance. In SEIRA experiments the enhanced vibrational signal scales with the square of the electric field, and it is highly located around the tip ends of nanoantennas and into the gaps. To quantify the sensitivity of SEIRA different parameters are used like the number of molecules from which the enhanced vibrational signal arises (both for a single nanoantenna and for the array of NAs). A further commonly used definition of SEIRA sensitivity is the enhancement factor (EF), which relates the enhanced signal strengths to standard IR techniques (transmission, reflection), which takes into account also the different geometry between the nanostructures and, consequently, the different distribution of molecules on the illuminated area. It is 
defined as follows [214]:

$$
E F_{S E I R A}=\frac{I_{S E I R A}}{I_{0}} \frac{A_{0}}{A_{S E I R A}}
$$

where $I_{S E I R A}$ is the enhanced and $I_{0}$ is the unenhanced signal intensity. Furthermore, $A_{0}$ is the area of the NAs exposed to the incident field and $A_{S E I R A}$ is the effective area of NAs on which the enhanced field is located. The enhanced SEIRA signal mainly originates from the molecules located in the antenna hot-spots, e.g. the tip ends for a linear antenna. On the basis of this finding, a commonly used approximation for the active area is the surface of the tip ends.

Molecules are therefore intrinsic and integral component of SERS/SEIRA and for an accurate theoretical treatment it is mandatory to include their interaction with the MS and to consider the electronic properties of the corresponding adsorbate, which is reported as chemical enhancement [215]. For this reason, the comparison between structures having different characteristics is often performed by taking into account also the device sensitivity in terms of limit of detection (LOD). The LOD (typically expressed in units of concentration) describes the smallest amount of analyte that can be reliably detected by a specific measurement process. However, also LOD is influenced not only by the substrate, but also by the chemical properties and the structure of the molecules [216]. For this reason, even though EF and LOD values appear as immediate terms of comparison between different PMSs, their absolute value should always be related to the nature of the targeted analyte and its chemical characteristics.

In the last decades, great efforts have been carried out to develop several substrates suitable for vibrational spectroscopies. In both techniques, preliminary results have been obtained with rough metal films like silver or gold [217-219]. Obviously, the enhancements that can be obtained by employing the roughness of metal films are low and, above all, not predictable nor reproducible, since a strong dependence of SERS/SEIRA effect on the surface morphology of the substrate subsists [220,221]. This dependence causes a variability also in the position of the plasmonic hot spots, so that the localization of target molecules in correspondence of the increased local fields becomes too fortuitous, especially for low concentrations of the analyte. For this reason, a great effort has been performed to develop fabrication techniques able to reduce at acceptable values the above mentioned variability (lower than 10\%), thus strongly reducing the reproducibility issues [222]. For vibrational spectroscopy applications, a lot of substrates have been developed so far; one of the most studied consists of the aggregation of metallic nanoparticles, like nanostars [223] or coreshell nanostructures [224], typically fabricated with bottom-up fabrication processes. These structures offer the possibility to achieve very strong hot spots thanks to the small nanogaps (below $10 \mathrm{~nm}$ ) between the single nanoparticles; on the other hand, a strict control on the nanogap arising from the synthesis of this kind of aggregation can be challenging. Instead, PMSs that are usually constituted by ordered array of metallic nanoantennas (NAs) let to achieve tailored plasmonic resonances in the desired region of the electromagnetic spectrum. Indeed, in order to reach the maximum efficiency, the plasmonic resonance must be finely tuned to the Raman scattering wavelength for SERS sensing [225], or to the maximum absorption wavelength of the target molecule 


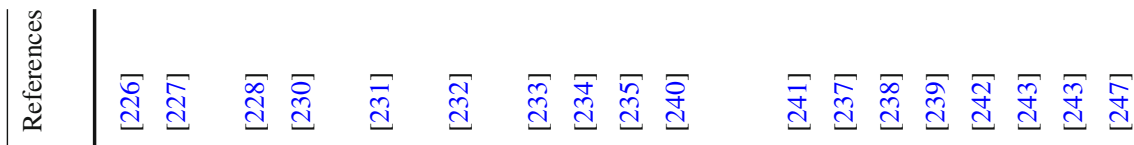

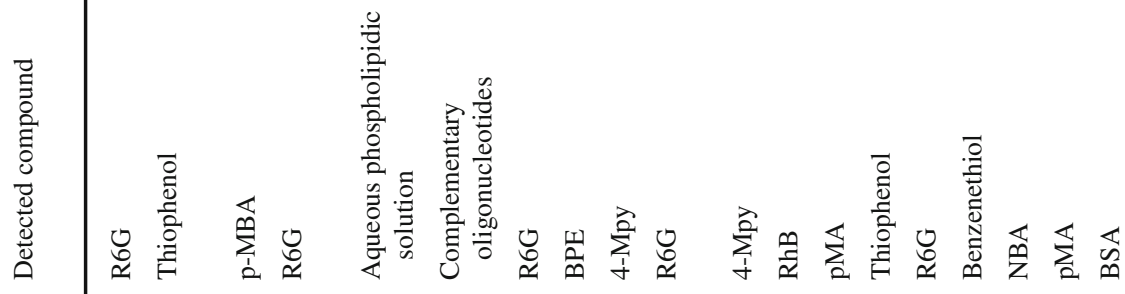

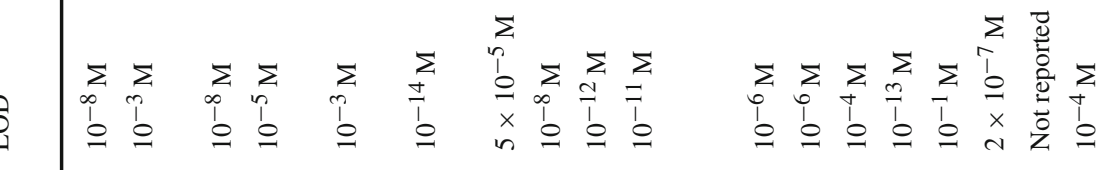

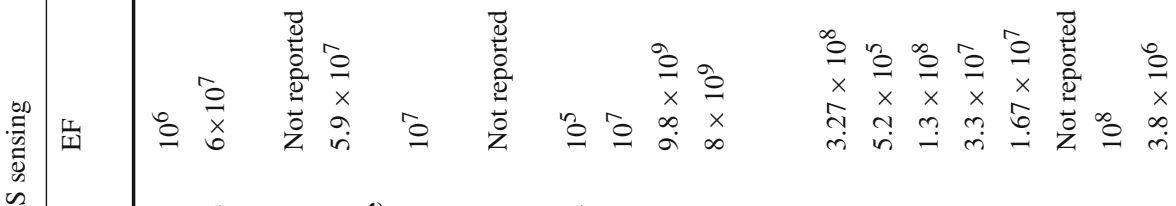

药

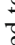

$\frac{7}{2}$

$\stackrel{\leftrightarrow}{\mathscr{2}}$

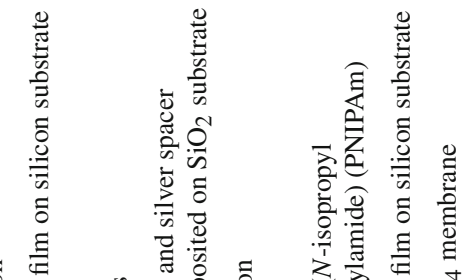

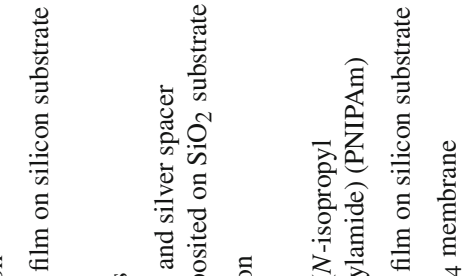

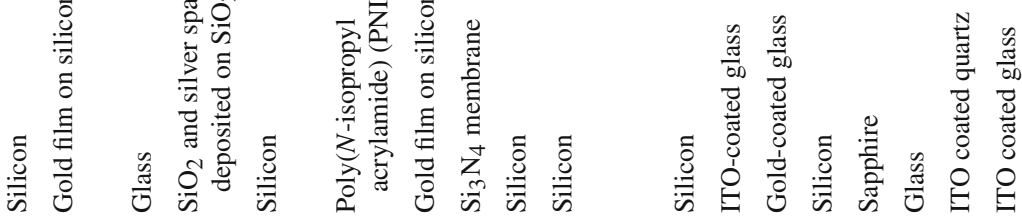

:

ग

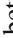

范

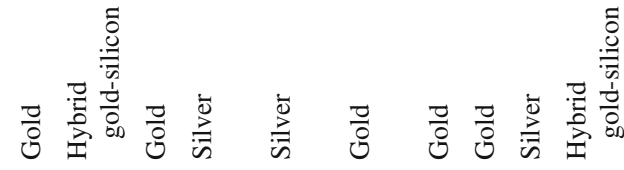

丞

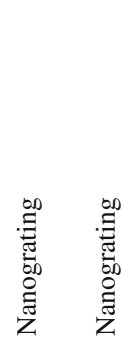

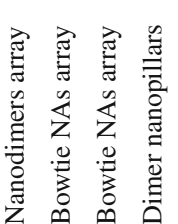

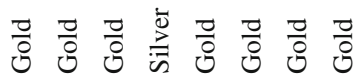

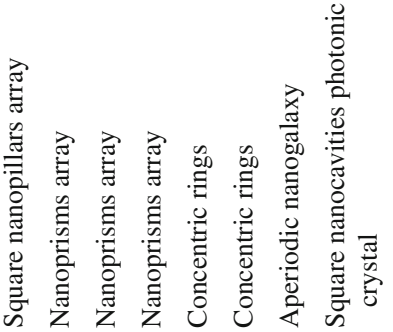


for SEIRA sensing [213], as it has been highlighted in the EFs definitions of Eqs. 9 and 10. Moreover, the gap between each nanostructure is well determined, ensuring a very good localization of the plasmonic hot spots. Thanks to the recent advancement in micro- and nanofabrication methods, it has therefore been possible to develop a large variety of PMSs for SERS/SEIRA sensing purposes in order to exploit the advantages offered by the different arrangements of NAs.

\subsection{SERS experiments}

In Table 1, some of the latest results for SERS biological sensing have been reported. This table does not aim to be exhaustive, but rather to show some of the MS geometries that have been considered so far in the design of SERS substrates. Table 1 has been organized as follows: in the first column it is reported the nanostructure geometry, followed by the description of the plasmonic material and the substrate on which the NAs have been fabricated. Then, the obtained EF and LOD for the detection of the chosen compound have been reported. For example, nanodisks or nanoholes arrays can be fabricated with high throughput and high reliability showing good results both in terms of EF and LOD [226-228]. The performance comparison in the detection of small molecules of thiophenol using hybrid gold/silicon nanodisks array [152] and gold nanohole array [228] is reported in Panels a and b of Fig. 11 respectively. Array of plasmonic nanoholes have attracted great interest thanks also to the possibility to achieve high EFs due to the coupling of two different plasmonic effects, SPP and LSP [229]. By introducing also a Fabry-Perot cavity under the nanohole array it is possible to realize selective SERS enhancements due to the fine tuning of the achieved multiple resonant device, as experimentally shown in Ref. [230] for a silver nanohole array optimized for the detection of Rhodamine 6G (R6G) molecules. Nanogratings, instead, have shown the possibility to enable multiwavelength detection when a gradient in the grating width is introduced, enabling the MS to support multiple resonances [231], together with long-range sensing due to their large area coverage [232]. The effect of the hybridization of localized plasmonic modes with the image modes in a metal film has been studied to improve the intensity of SERS signals, realizing arrays of different plasmonic nanodimers on gold metal film [233]. A comparison between different nanodimers geometries (circular, rectangular and bowtie) has been performed, showing that, although all the experimental EFs were of the order of $10^{5}$ for the detection of R6G molecules, a bowtie configuration should be preferred, since this geometry shows the strongest electric field enhancement. This enhancement is due to the combination of both hot spot localized into the gap between NAs and the enhancement due to the bowtie sharp edges [234], and can be further improved by elevating the NAs on silicon pedestals, thus forming a Fabry-Perot cavity that allows to achieve high EFs $\left(\simeq 10^{10}\right)$ releasing the need of sub-10 nm gaps between NAs tips [235]. The combined effect of the field enhancement due to the gap between NAs and the sharp edges of NAs, namely the lightning-rod effect [236], can also be exploited in plasmonic nanoprisms array configuration. Nanoprisms, in contrast to bowtie NA dimer structures, can lead to a more compact configuration in terms of both plasmonic hot spots and NAs tips, thus enabling high sensitivity detection [237-239]. In addition 

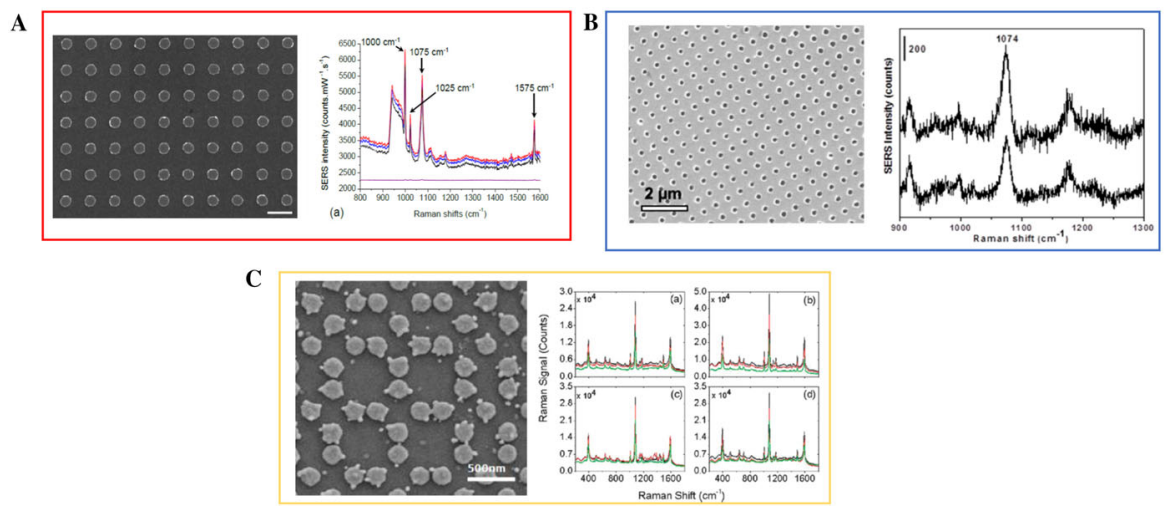

Fig. 11 SERS sensing with plasmonic metasurfaces. a (red box) A SEM image (on the left) and the SERS signals (on the right) of hybrid gold-silicon nanodisks array fabricated on a gold film in detecting thiophenol molecules. Reprinted with permission from [227]. b (blue box) A SEM image (on the left) and the SERS signals (on the right) of a gold nanohole array in detecting para-mercaptobenzoic acid molecules. Reprinted with permission from [228]. c (yellow box) A SEM image (on the left) and the SERS signals (on the right) of an aperiodic nanogalaxy gold array for detection of p-Mercaptoaniline. Adapted with permission from [233]. Copyright 2009 American Chemical Society

to elevated NAs, also arrays of plasmonic nanopillars have been considered for SERS sensing, since this configuration reduces the interference of the electromagnetic field with the substrate and provides, at the same time, a tridimensional sensing environment $[240,241]$. Also structures without sharp edges have shown satisfactory results in terms of both EF and LOD; in particular, metallic concentric rings have been considered due to their capability to focus light, allowing therefore to obtain a high spatial control over SERS signal intensity [242,243]. Finally, even though periodic arrangements of metallic NAs constitute the most employed substrates for SERS sensing, they are not the unique possibility. Indeed, aperiodic plasmonic structures offer a higher flexibility in engineering and tailoring plasmonic resonances and optical responses of the MSs thanks to the relaxing of the periodicity constraint [244]. As an example, in panel c of Fig. 11 the SERS detection of a monolayer of p-mercaptoaniline (pMA) molecules [245] using an aperiodic nanogalaxy of gold nanocylinders is shown.

All the reported results show how the different arrangements and geometries of PMSs can lead to very significant results for SERS sensing. Indeed, as it can be seen, most of the structures resumed in Table 1 show EFs higher than $10^{7}$, that in many cases is enough to perform single molecule analysis [246].

\subsection{SEIRA experiments}

Table 2 is organized as Table 1 and aims to resume some of the latest results achieved with different MSs in SEIRA sensing experiments. Ordered array of metallic nanorods is one of the most studied and employed MS, where the field enhancement is mainly located on the NAs tips and can be further raised thanks to the collective contribution of the periodic arrangement. These structures showed EFs around $10^{4}-10^{5}$, allow- 


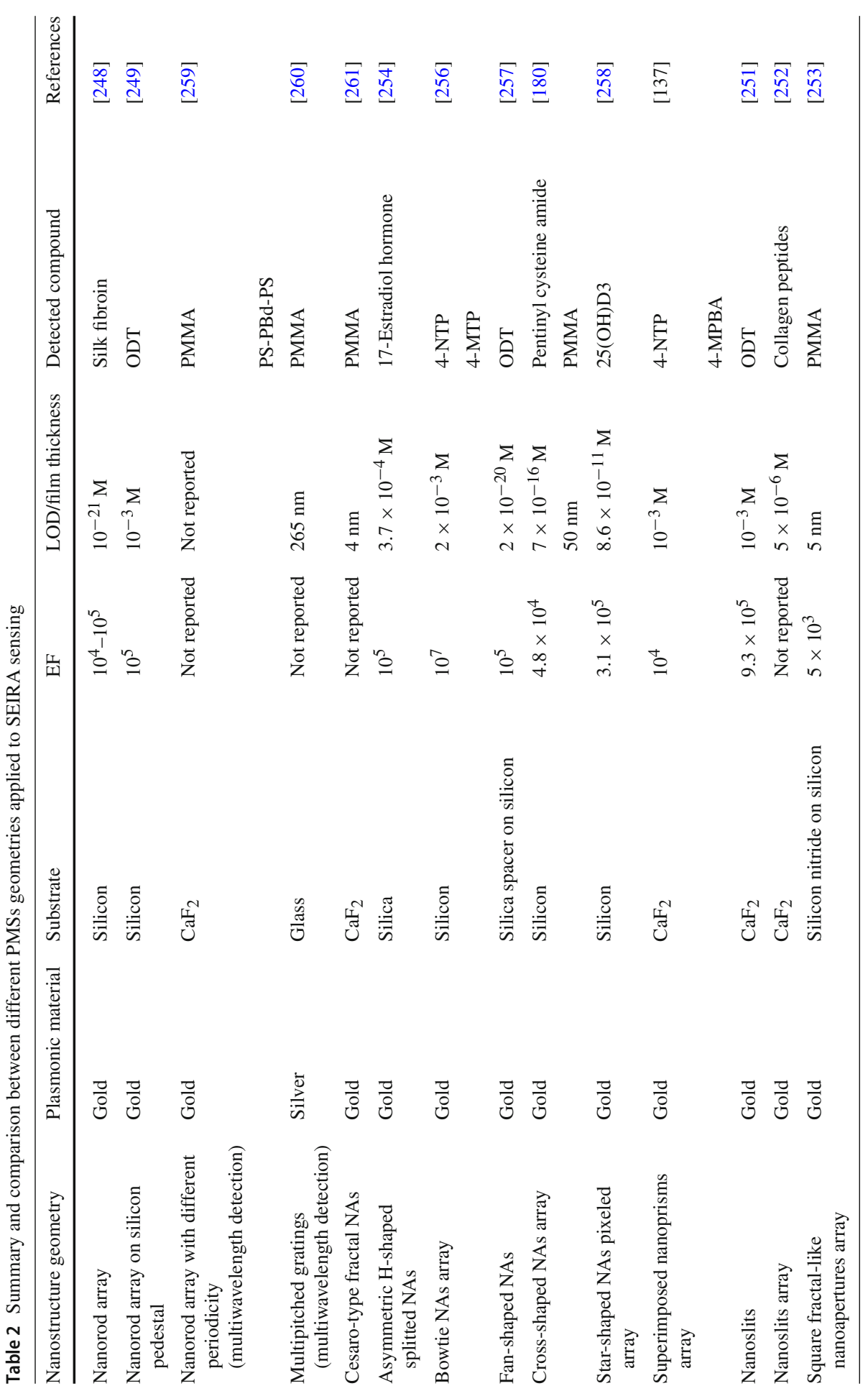


ing the detection of zeptomolar concentrations of proteins [248,249]. Following the Babinet principle [250], also inverse structures like nanoslit arrays have been proposed and tested. Indeed, this kind of structure is able to achieve higher near-field enhancement due to the very strong hot-spot localized in the nanoaperture [251] and results are particularly appealing for the specific chemical identification of particles with sub-micrometer size, like collagen peptides [252]. Moreover, nanoslits also show a reduced sensitivity to the change of RI of the surrounding medium as compared to NAs, thus enabling the possibility to perform an in vitro monitoring of structural changes of molecules [252]. In addition, square fractal-like nanoapertures in a gold film have been considered; they show an $\mathrm{EF} \simeq 10^{3}$ and a refractive index sensitivity of more than $2 \times 10^{3} \mathrm{~nm} / \mathrm{RIU}$ [253]. The combined effect of NAs sharp edges and nanoslit hot-spots has been adopted using an asymmetric $\mathrm{H}$-shaped splitted nanoantennas array, in detecting $M$ concentrations of 17-Estradiol molecules [254]. The sharp edges of NAs and the narrow slit between them allows to achieve high $\mathrm{EF}\left(\simeq 10^{5}\right)$ and good sensitivity, up to $10^{3} \mathrm{~nm} / \mathrm{RIU}$, but the asymmetry of the structure induces some limitation in SEIRA analysis. Indeed, considering for example nanorod configuration, light perpendicularly polarized with respect to nanorod axis does not excite any resonance [255]. Bowtie nanogapped antenna arrays [256], like those shown in panel a of Fig. 12, and fan-shaped nanoantennas array [257] again combines the NAs sharp edges with a nanogap. Such configuration guarantees values for EFs up to $10^{7}$ and the capability to detect monolayers of molecules. However, there is still a dependence on the polarization of the incident light, even if lower as compared to the nanostructures presented hitherto. Analyte orientation on the nanoantennas is randomly distributed, so nanoantennas configurations that are polarization independent could introduce some advantages for SEIRA sensing. For this purpose, cross-shaped gold nanoantennas geometry has been adopted; thanks to the combination of the NAs sharp edges and the collective response of the plasmonic array a value for EF of $5 \times 10^{4}$ and a capability to detect compounds down to femtomolar concentrations have been achieved [180]. The multiplexing capability of SEIRA spectroscopy has been shown to detect extremely low concentrations of hormone 25(OH)D3 (pM concentration) with a MS made of star-shaped gold nanoantennas [258]. Thus, preserving the independence from light polarization, the MS has been arranged in several pixels each having resonance in different part of the spectrum allowing a multiwavelength detection, as shown in panel $b$ of Fig. 12. The possibility to perform a multiwavelength detection in both fingerprint and functional group regions has gained a rising interest, so that several structures have been proposed to this aim by exploring the combination of arrays with different periodicity as shown in panel c of Fig. 12 [259]. A multipitched metal-coated diffraction grating [260] has been used to detect the C-H stretching modes in a 262 nm thick poly(methyl methacrylate) (PMMA) film deposited on it. Cesaro-type gold fractal nanoantennas have been used [261] to detect the presence of a very thin PMMA molecular layer down to the thickness of $4 \mathrm{~nm}$.

Moreover, due to the complementarity of SERS and SEIRA sensing, very interesting results have been achieved [262] with a composite array of nanoprisms and triangles, as shown in panel d of Fig. 12. The superposition between these structures makes the proposed MS suitable for both SEIRA and SERS sensing, with EF of $10^{4}$ and $10^{5}$, respectively, paving the way for multispectral detection of molecules. All the proposed 
A
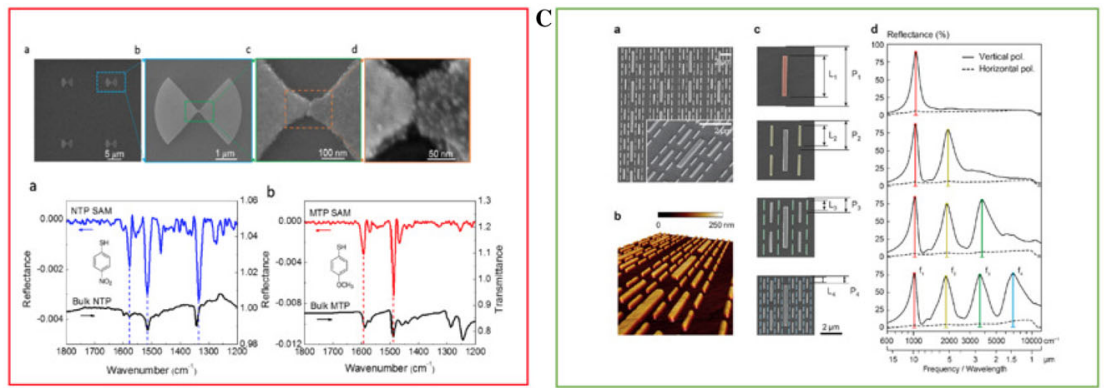

B
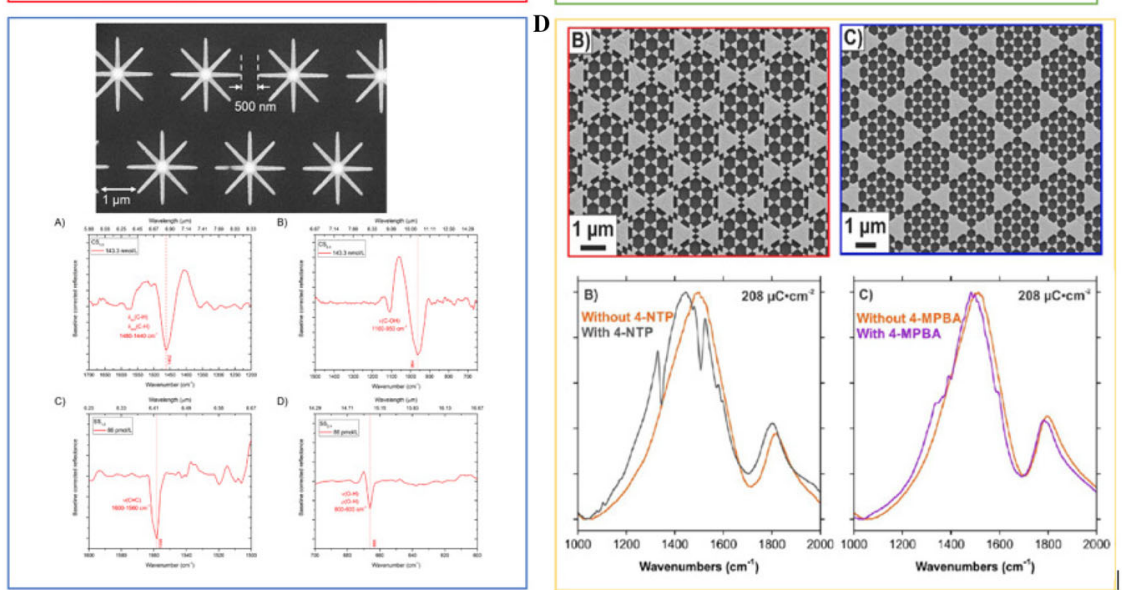

Fig. 12 SEIRA sensing with plasmonic metasurfaces. a (red box) A SEM image (on the top) and the SEIRA signals (on the bottom) of gold bowtie nanoantennas for SEIRA detection of 4-nitrothiophenol (4-NTP) and 4-methoxythiolphenol (4-MTP) molecules. Adapted with permission from [256]. Copyright 2017 American Chemical Society. b (blue box) A SEM image (on the top) and the SEIRA signals (on the bottom) of star-shaped NAs array in detecting pM concentrations of 25(OH)D3 vitamin. The device has been organized in pixels having different NAs arrangements to allow multiwavelength detection. Adapted with permission from [258]. c (green blox) The SEM image (on the left) and the SEIRA signals (on the right) of a multiresonant structure rising from the combination of nanorod arrays with different periods. This metasurface is able to perform SEIRA sensing both in fingerprint region and in functional groups region of the spectrum. Reprinted with permission from [259]. Copyright 2018 American Chemical Society. d (yellow box) The capabilities composite array of nanoprisms and triangles in SEIRA detection of 4-nitrothiophenol (4-NTP) and 4-methoxythiolphenol (4-MTP) molecules. Adapted with permission from [262]. Copyright 2016 American Chemical Society

structures for SEIRA sensing present very high field enhancement values, and, together with SERS sensing, can lead to a complete analysis of the target molecule.

SERS and SEIRA have become mature surface-enhanced/modified vibrational spectroscopic techniques over the last decades. The electromagnetic enhancement has been identified theoretically and experimentally as the dominant mechanism. Nanofabrication methods (described in the next section) have also matured considerably during the last decade. Solid planar/2D SERS substrates can be reliably generated by physical top-down approaches with high precision. One of the major driving forces behind the design of highly sensitive and robust SERS/SEIRA substrates with precisely tuned 
plasmonic properties is devoted to a full understanding and control over the formation of the hot-spot sites. Substantial work remains in order to determine the prominence and estimate the magnitude of the chemical contribution to the enhancement. Finally, the classical/macroscopic description of these effects is not always sufficient and a more sophisticated quantum mechanical/microscopic treatment could be required.

\section{Fabrication methods of plasmonic metasurfaces}

Improvements in fabrication methods have been allowing the realization of cuttingedge metasurface devices. With the full understanding of the optical properties, and the constant improvement of the nanotechnologies employed in the realization of metasurfaces, it is possible to face challenges in several fields, from bio-chemical sensing and imaging in human health, to optoelectronics for energy harvesting and telecommunications. Plasmonic metasurfaces could be implemented also in non-conventional substrates such as optical fibers [263-266].

The methodology chosen for PMS manufacturing has to satisfy essential process requirements, such as resolution, productivity, reproducibility and cost efficiency. The most used fabrication technology in semiconductor integrated circuits is photolithography, that allows high throughput at the micro- and nanoscale. With this technique, the micro- and nano-patterns are transferred from photomasks to photoresists after the exposure and subsequent development process. Final nanostructures are formed on the substrates after a series of processes requiring thin film material depositions, etching and lift-off procedures. Unfortunately, this technique suffers of a very limited spatial resolution due to diffraction limit of light. For this limitation, photolithography is not suitable for realizing high quality nanoscale metasurfaces based on hierarchical 2D and 3D nanostructures. Several types of alternative techniques have been employed, each with its own advantages and disadvantages. A possible classification consists of top-down and bottom-up techniques. Some methods (i.e., nano-sphere litography) conjugate both approaches. The top-down process starts from a suitable large material and then, through high-resolution techniques, creates smaller structures. This methodology comprises electron beam lithography, focused-ion beam lithography, laser lithography and nanoimprint lithography. In the bottom-up approach, small components of atomic or molecular dimensions self-assemble together, according to a natural physical principle or an externally applied driving force, to give rise to larger and more ordered systems.

In this section, the aforementioned techniques will be illustrated reporting characteristics, advantages and drawbacks, citing some examples of plasmonic MSs found in recent literature.

\subsection{Electron beam lithography}

Electron-beam lithography (EBL) employs a modified scanning electron microscope (SEM) to scan a focused beam of electrons able to write a custom nanoscaled pattern on a surface covered with an electron-sensitive film called resist. The electron beam 
changes the solubility of the resist, enabling selective removal of either the exposed or non-exposed regions by immersing it in a solvent (developing). Electron-beam lithography systems can be classified according to both beam shape and beam deflection strategy. Newer systems operate with shaped beams, which can be deflected to various positions in the writing field (vector scan mode). The system uses a blanker to switch on and off the beam. After the pattern is impressed onto the resist (positive or negative tone), the exposed portion of the resist is removed (if the resist is a positive tone) using a developer. This leaves a nanostructure pattern that can be further processed. In EBL a deposition process (such as evaporation or sputtering) together with a lift-off procedure is used to build up structures at the nanoscale (see process schematization in Fig. 13). Another manufacturing process involving EBL system together with dry or wet etching is usually exploited to create mainly dielectric nanostructures, masks and molds for other fabrication techniques, such as nanoimprint lithography. EBL needs a long processing time to realize the desired pattern onto the electron resist, because the electron-beam is focused on a single point of pattern at a time. This characteristic together with the high operating costs of this method [267] limits its use for commercial purpose where high throughput for large-scale production and large area device fabrication are necessary. The main advantages of EBL technique is the excellent flexibility and the high resolution (about $10 \mathrm{~nm}$ ) [268] that allow realizing nanostructures with complex geometric shapes [269]. Thanks to these characteristics, this technique is broadly employed in the fabrication of cutting edge nanostructures also on non-conventional substrates, such as the tip of an optical fiber [263,264,266].

\subsection{Focused ion beam lithography}

Unlike EBL, focused ion beam (FIB) lithography consists in a one-step process, as illustrated in Fig. 13, and it is based on an ion beam (instead of an electron beam as in EBL process) that etches the sample surface. As EBL, also FIB is very advantageous in the fabrication of nanostructures because of the intrinsic high resolution of the process. FIB source is based on heavy ions $\left(\mathrm{He}^{+}\right.$or $\mathrm{Be}^{+}$or $\left.\mathrm{Ga}^{+}\right)$for direct writing of the nanopatterns, ensuring a stable and fine beam spot [270]. Often the FIB column is coupled with a SEM in order to guarantee vision and writing at the same time. Employed electrons, in EBL systems, have larger de Broglie wavelengths and enlarged beam sizes due to scattering, while in FIB, the heavier ions have less penetration depth, which decreases the aspect ratio of the nanostructures [271]. The FIB exposure sensitivity is higher than the EBL one [272], involving energies typically around 100$200 \mathrm{kV}$ [273]. Similar to EBL process, also for FIB procedure the throughput and large area fabrication is a challenge for commercial production. But similar to EBL, the FIB approach could be employed in the fabrication of high-resolution metasurfaces [270] also on the tip of an optical fiber [265].

\subsection{Nanoimprint lithography}

Nanoimprint lithography (NIL) establishes a good compromise between resolution and cost in large-area patterning of nanostructures [149] [150]. NIL is based on the 


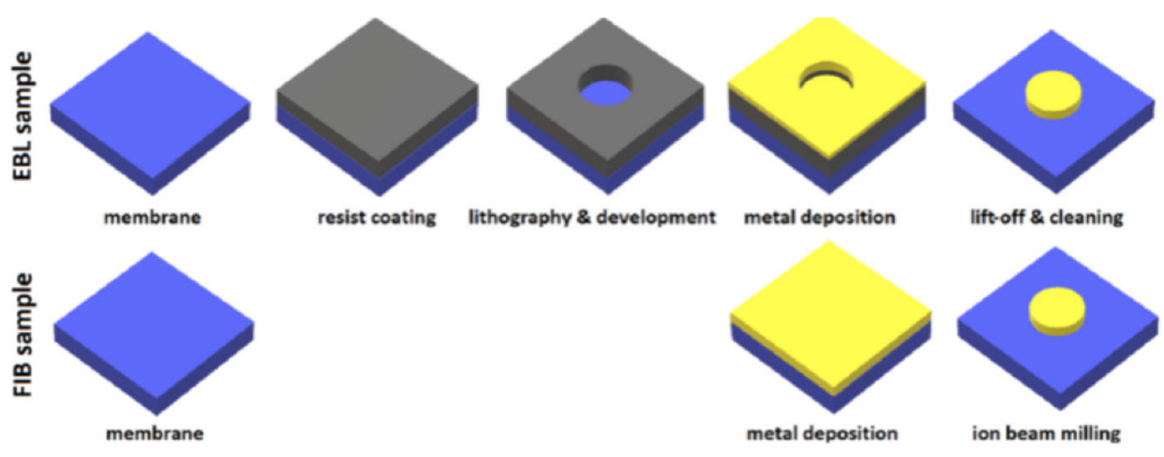

Fig. 13 Schematic overview of the individual steps in the fabrication process of EBL and FIB antennas. Reproduced with permission from Ref. [274]

application of molds (stamps), typically prepared using EBL and reactive ion etching (RIE) techniques, to realize nanostructures with the following steps: (1) the mold with the desired pattern is mechanically pushed into the imprint resist that is coated on a substrate, in order to transfer the design in the specific polymer; (2) successively the resist is cured and the stamp removed, leaving the nano-pattern on the substrate. The stamp pattern size defines the minimum feature size of the nanostructure pattern. Two NIL procedures have been developed, depending on the type of resist curing: thermal NIL and UV NIL [275]. The first one operates with a thermoplastic resist, while, in UV NIL systems, a photocurable polymer is employed. UV-NIL is carried out at a constant temperature and an extremely low pressing pressure, which is desirable for precise patterning required in lithography. However, when UV NIL is performed in air, the low pressing pressure leads to the formation of air bubble defects [276], not observed in the thermal process, that employs a high pressing pressure, not always advisable. The air bubbles formation could be solved by applying a vacuum atmosphere [277], which would affect the cost-effectiveness of the nanoimprint systems. Also with thermal NIL it is possible to obtain high resolution obtaining flexible plasmonic sensing platform as verified also by Farcau et al. in Ref. [278]. In this work, the authors employ a NIL process (schematic steps shown in Fig. 14a) to realize nanopost-shells arrays with periods of $300 \mathrm{~nm}$ and $400 \mathrm{~nm}$ (see Fig. 14b). The main applications of NIL are in optical storage devices, hard disk media devices, light emitting diodes, biosensors, and microfluidic devices [279].

\subsection{Laser lithography}

Laser lithography is a mask-less technique used for the realization of devices on large-scale production. There are two different laser lithography approaches, both of them capable of fast and low-cost processing of nanostructures: laser-direct-write (LDW) lithography and laser-interference lithography (LIL). LDW lithography uses computer-controlled optics to project the desired nano-patterns directly onto the photoresist. This technique allows working materials that are hard to machine and also generating 3D surface profiles by varying the energies of the employed laser. LIL 


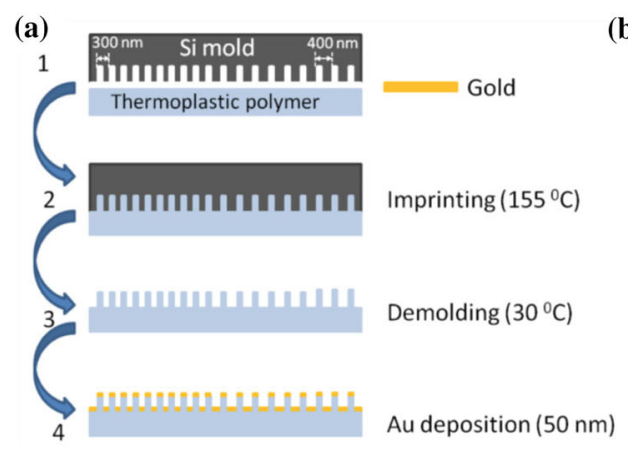

(b)

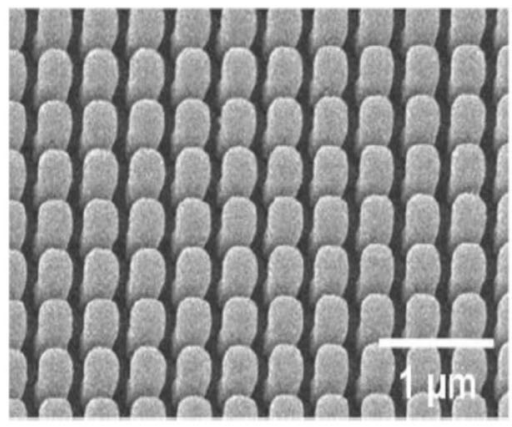

Fig. 14 a Schematic steps of the NIL process used for realizing gold nanopost-shell arrays; b SEM image of the Au nanopost-shell arrays fabricated by NIL with period of $400 \mathrm{~nm}$. Adapted and reprinted with permission from [278]

Fig. 15 SEM image of the array of holes recorded in the Au film after the lift-off of the resist. Reprinted with permission from [281]

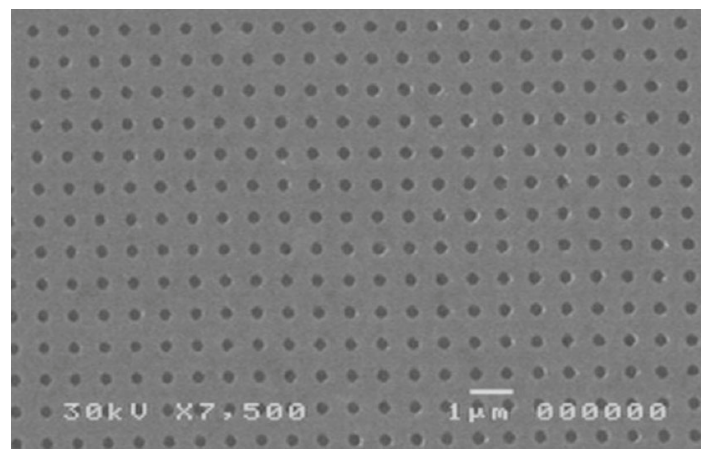

is a mask-less photolithographic technique used for the fabrication of nanostructures through the superposition of multiple laser beams onto a photoresist [280]. As reported recently by Valsecchi et al. [281], this method is well suited to produce on large scale well-resolved plasmonic nanostructures such as gold nanoholes (see Fig. 15) for sensing applications. Patterns of different sizes and shapes can be easily obtained by the interference of multiple laser beams. The resolution is only limited by the wavelength of light being used during the pattern transfer [282]. Indeed, not all shapes can be patterned, and the minimum period of nanostructures is limited to the half of the wavelength of light. This requires the use of deep ultraviolet (UV) light for smaller features [283], which makes LIL very expensive. For large exposure areas, the laser source should have a long coherence length and, for higher resolution [284], lower wavelengths of light are needed. The light source specifications required for the desired patterns makes the LIL challenging for large-scale fabrication.

\subsection{Bottom-up techniques}

Self-assembly lithography is an efficient and facile patterning method for the production of various large-area nanostructures, including chains, sheets, and 3D con- 
structions, where the system is spontaneously assembled by intermolecular balance of attractive and repulsive forces [285].

Self-assembly is realized by exploiting the chemical and physical properties of nanoparticles, surfaces, and their interactions [286]. The principle on which this technique is based is the reduction of the free energy necessary to achieve the local equilibrium of the constituent components. The local equilibrium can be favored or externally modulated by exploiting direction fields and/or confinement effects. In direct self-assembly technique external fields and templates are used to favor a selective grouping of colloids and spatial order. The main method for the bottom-up manufacture of colloidal metasurfaces is template-assisted colloidal self-assembly that uses capillarity and capillary forces to guide the process on the interface of the templates, which consist of objects with modified surfaces that provide sites of selective bonds. Colloidal self-assembly has some advantages over the top-down manufacturing methods described above. This technique allows obtaining well-defined plasmonic nanoparticles in terms of shape and crystallinity, which ensures high optical quality at the level of a single nanoparticle. Furthermore, this template-based approach is scalable and energy efficient.

EBL, FIB and LIL are characterized by a low throughput and high construction costs. These issues make these top-down techniques not the best choice for large-scale manufacturing. For these reasons, some bottom-up techniques have been evaluated to solve these realization challenges. Among these, NanoSphere Litography (NSL) has been in-depth investigated [287,288]. The NSL is a high throughput and inexpensive technique, which allows creating two-dimensional and three-dimensional nanostructures. Using this technique, the spherical colloidal solutions are transferred onto a substrate and dried to form a closed hexagonally packed monolayer (HCP) [289]. This method is usually adopted for polymeric coatings, such as dip coating [290], spin coating [291] and Langmuir-Blodgett [292]. The obtained monolayer is used as a sacrificial mask for the subsequent formation of arrays of nanostructures [293]. With this technique it is possible to produce large and long-range ordered gold nanoring array patterns in a controllable fashion [293] as can be seen in Fig. 16. As shown in Fig. 16a-c, it is possible to obtain gold nanoring array patterns with slightly different parameters (periodicity, radius, gold thickness) on a large scale (see Fig. 16d, e). The plasmonic material is deposited in the interstices of the HCP beads and subsequently, through a lift-off process, the sacrificial mask is removed leaving an ordered array of nanostructures on the chosen substrate. This technique is limited in its versatility: the HCP monolayer used as a lithographic mask does not allow significantly varying the shape and dimensions of the desired geometries.

In addition to the techniques just illustrated, another one, named Dip PeN lithography (DPN), can be employed either as top-down or as bottom-up process. DPN is a direct, AFM-based write method, invented by Mirkin's group and used for top-down and bottom-up soft-lithography applications [294]. In top-down modality, the tips are used to deliver an etch resist to a surface, which then undergoes a standard etching process [295]. In bottom-up applications, the material of interest is delivered directly to the surface via the tips. This technology was developed to pattern inks including organic molecules, colloidal particles, metal ions and biological polymers on various substrates (metals, insulators and semiconductors) [296]. DNP lithography guarantees 

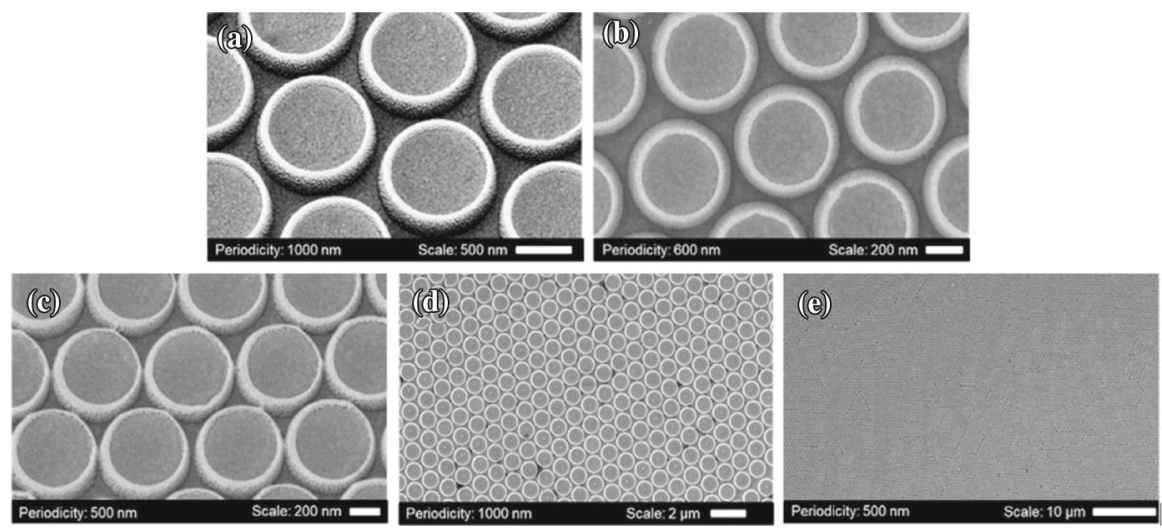

Fig. 16 a SEM image of the Au nanoring pattern (periodicity: $1000 \mathrm{~nm}$, outer radius: $920 \mathrm{~nm}$, thickness: $75 \mathrm{~nm}$, and height: $120 \mathrm{~nm}$ ); b pattern (periodicity: $600 \mathrm{~nm}$, outer radius: $500 \mathrm{~nm}$, thickness: $50 \mathrm{~nm}$, and height: $120 \mathrm{~nm}$ ); c pattern (periodicity: $500 \mathrm{~nm}$, outer radius: $440 \mathrm{~nm}$, thickness: $30 \mathrm{~nm}$, and height: 120 $\mathrm{nm})$; d, e The Au nanoring array patterns with large-scale area. Adapted and reprinted with permission from [293]

high resolution (tens of nanometers), as reported by Mirkin in 2007 [297] for patterning of alkylthiols on gold substrate. The DPN technique is unfortunately expensive, with a limited number of usable materials and has a low production yield, although in 2006 Salaita et al. [298] demonstrated the possibility to use parallel 2D DPN.

Finally, several hybrid patterning processes have been developed for the realization of more complex nanostructures. As depicted in Fig. 17a, hole-mask colloidal lithography and off-normal deposition are combined to fabricate directional metasurfaces with different tilted nanopillars (shown in Fig. 17b) [299]. Hole-mask colloidal lithography using roller template stripping has been proposed to pattern subwavelength metallic nanoholes, nanodisks, nanowires, and pyramids by taking advantage of plastic materials with flexible and stretchable properties [300]. First, the metal film is deposited on a poly(methyl methacrylate) (PMMA) substrate with polystyrene nanospheres, and then the nanospheres are removed by tape stripping to form the hole-mask. Finally, the tilted nanopillars are obtained by deposition of the metal with a tilted angle and subsequent removal of the PMMA.

\section{All-dielectric photonic structures}

Optical devices, metasurfaces and sensors at the nanoscale are often associated with plasmonic structures made of noble metals such as gold or silver in which resonance tailoring and electric field confinement down to volumes comparable with molecular sizes have governed a plethora of fundamental research and cross-linking applications in many fields, from quantum optics, ultrasensitive physical and chemical sensing, to novel investigation tools for advanced biochemistry and medicine. However, in the visible range where detecting schemes are more efficient and footprints are smaller, 
(a)
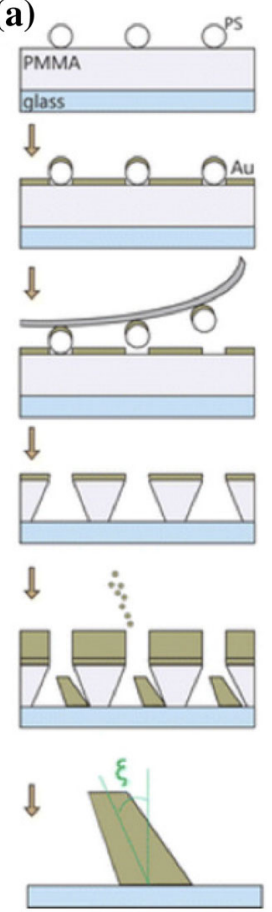
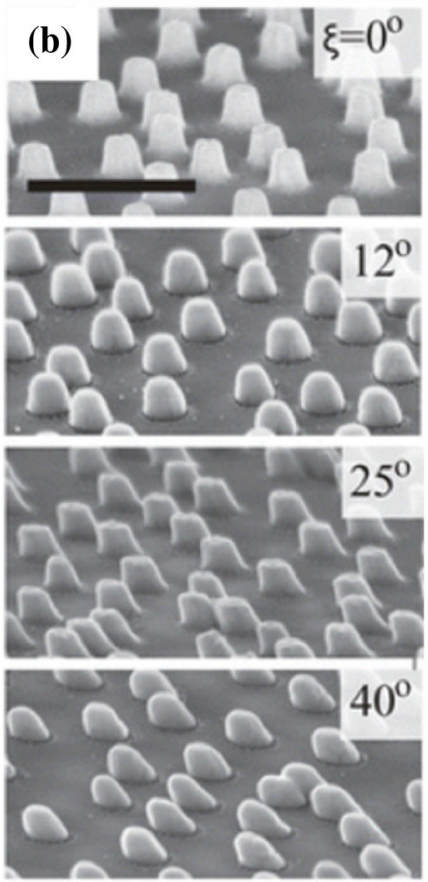

Fig. 17 a Fabrication steps of tilted nanopillars using hole-mask colloidal lithography; b SEM images for different tilting angles (the scale bar corresponds to $500 \mathrm{~nm}$ ). Adapted and reprinted with permission of [299]

plasmonic devices are intrinsically limited by ohmic losses of metals and incompatibility with complementary metal oxide semiconductor fabrication processes.

A new challenge of current photonic research aims at pursuing plasmonic capabilities through dielectrics, in particular high-refractive index materials that can sustain multipolar electric and magnetic resonances, finding unexpected applications in energy conversion, nonlinear photonics, lasing and sensing. At the same time, the fundamental physics of light interaction with dielectric materials has recently reached a higher level of maturity, with the discovery of topological phases and further understanding of spin-orbit angular momentum coupling of light, which has been increasing the capabilities in light control and manipulation. Large efforts have been dedicated in the last few years to the engineering of radiation-less resonances in dielectric materials since the associated large radiative $Q$-factor can bring great advantages for what concerns optical near-field enhancement and sensing due to perturbation processes. These properties are at the foundation of the cutting-edge applications herein reviewed. In the following section, we will first discuss some basic concepts pertaining this topic with the aim to provide a very general framework to the interested reader. Then, we will deepen the discussion about main non-radiating resonances. 


\subsection{Single and multiple scattering in dielectrics}

It is interesting to analyze the origin of resonances in dielectric MSs for the two cases of single scatterer whose dimensions are comparable with or much smaller than the wavelength. In the first case of single scatterer structures of the order of a cubic wavelength and substantially diffraction limited, the resonance is due to a stationary wave produced by waves oscillating between the resonator walls and exchanging energy among electric and magnetic fields, in a way similar to a mass suspended to a spring in which there is a continuous transfer between potential and kinetic energies. The self-sustaining mechanism can lead to very high $Q$-factor values over six orders of magnitude [301]. In this regard, we remember that the $Q$-factor of a resonance for a harmonic wave can be defined, with a factor $2 \pi$, as the ratio between the temporal average of the energy accumulated in the cavity and the losses in a wave cycle. In the second case of scattering structures significantly smaller than the wavelength, well below the diffraction limit, the mechanism of exchange between electric and magnetic field energies can not occur. These subdiffractive structures behave as poorly efficient antennas and the $Q$-factor does not exceed the enhancement of an order of magnitude ( $Q \simeq 10$ ). This significantly differentiates the two resonance regimes, characterized by a strong difference in the $Q$-factor values. In metal structures, the plasmons are confined in an extremely small volume, much smaller than $\lambda^{3}$. In this case, even if the value of $Q$ is limited, the ratio between $Q$ and the interaction volume $V$ [206] is large, which ultimately determines the decay rate and the so-called Purcell effect.

Analytical expressions can also be obtained for the polarizability of some regular objects (such as the well-known Mie theory for spheres but also ellipsoids [302]) and some hybrids. In general, an exact analytical model is not possible and, even for finite size cylinders, numerical calculations must be used to recover quantities such as the scattering cross section [303], showing that some non-radiating modes appear to be an interference mechanism due to the strong coupling between Mie-like and FabryPerot-like modes. The approximation relies on the fact that the high refractive index used does not make such analysis rigorous for the optical and NIR wavelengths. When the single dielectric scatterer is periodically replicated in space, lattice resonances due to Bragg interference among Bloch waves occur. These resonances can be combined with resonances of the single scatterer giving rise to a wide range of scenarios [304]. Among the different possibilities, there are the interactions between the resonances of the single scatterer and the lattice resonances that can interfere, producing a Fano resonance when a zero and a pole of the scattering matrix approach each other. When the zero and pole merge on the real axis, a so-called bound state in the continuum (BIC) emerges [305], which will be discussed in more detail in Sect. 9. The scattering properties of so-called anapoles, another special case of nonradiating sources [306], will be discussed in Sect. 10.

\subsection{Dielectric plasmonics}

A parallelism exists between some relevant plasmonic properties of broad applications and similar behaviors that can be experienced in dielectric materials. Most of the 
plasmonic properties of metals, at visible or infrared wavelengths, comes from the resonances linked to the negative value of the dielectric function, which can be easily described by the Drude-Lorentz model [302]. On the other hand, it is nowadays well known that even structured dielectrics, such as certain photonic crystals, can have negative dielectric function values, and sometimes a negative refractive index [307,308]. For instance, it was shown experimentally [309] that a periodic dielectric structure suitably designed to have a negative effective refractive index in a desired wavelength range, can be described by means of a homogeneous dielectric function with a Lorentz resonator:

$$
\epsilon(\omega)=\frac{f \omega_{0}^{2}}{\omega_{0}^{2}-\omega^{2}+i \gamma \omega} .
$$

The above experiments were made in air for wave vectors above the light-line near the $\Gamma$ point. Analogous results were obtained with a prism set-up coupling of evanescent waves below the light line, measuring a dispersion typical of surface plasmons at the interface with a metal [310]. These measurements, carried out with KretschmannRaether configuration [311], confirmed the existence of surface waves at the external interface of dielectric photonic crystals with a negative effective permittivity, in strict analogy with surface plasmons. The surface wave nature is at the basis of most plasmonic applications, such as near field localization and enhancement, as well as sensing associated to the evanescent behavior of the field. Indeed, the strong enhancement of the EM field and its evanescent tails at the metal/dielectric interface is exploited in sensing small refractive index changes of the surrounding environment. As already mentioned, metal-based structures may have high absorption at optical frequencies. Local heating and heat dissipation broadens resonance profiles and may induce damages to the samples. It is therefore extremely interesting to design surface waves mimicking localized surface-plasmon resonances in terms of high-field intensity and spatial localization. In the following sections we will provide an overview of BIC and anapole resonances, which, as discussed, are providing a paradigm shift in nanophotonic applications of dielectric structures.

\section{Bound states in the continuum}

From being confined to the realm of theoretical physics since the pioneering study of von Neumann and Wigner in 1929 [312], BICs have been later theoretically and experimentally studied in many wave phenomena, from acoustics to optics $[313,314]$. In nanophotonics, the literature regarding BICs correlated phenomena has grown to testify an increasing interest in their understanding and applications [312,315-330]. One of the reason relies on the intriguing physics behind them, the potential associated with non-radiating sources [328] which BICs belong to, but also on the feasibility of their integration in nanophotonic devices and systems that could take advantage of new paradigms in enhanced light-matter interaction control for lasing [331-334], high harmonic generation [335-338], surface-enhanced fluorescence and Raman scattering [339-341], structured radiation and polarization engineering [342,343], spin-to-orbit coupling and transport [344,345], unprecedented waveguiding possibilities in core 
materials with refractive index lower than cladding's [346], high throughput sensing [326,327,330], and enhanced entangled photon pairs generation for quantum processing [347,348]. A recent and comprehensive review on photonic BICs can be found in ref. [349].

For what concerns the optical phenomenon, a BIC is a special mode having a frequency falling in the spectral range of the radiation continuum [322]. Although it shares same frequencies with loss channels, it does not couple with them and therefore it does not loose energy by radiating into the free space. Recent works investigated BICs in periodic structures, photonic crystal slabs, dielectric waveguides, and array of rods or cylinders [350-355].

Below the air-light line, i.e. the dispersion of free space radiation, the dispersion diagram of a photonic crystal slab or a periodic metasurface (with air or dielectric pillars) traces the frequency of modes sustained with specific momentum. The modes are confined inside the structure and as such have infinite radiative $Q$-factor, but only ideally since scattering and absorption losses reduce it to about ten orders of magnitude. When the frequency of the mode climbs over the light line, the radiation in the slab can escape into the free space and is termed leaky mode. Approaching a high symmetric point of the dispersion relation, such as the normal incidence ( $\Gamma$ point in the reciprocal space), the mode may inherit symmetries of the dielectric geometry that are not compatible with radiation. One of the most important demonstrations of photonic BICs has been done with holey photonic crystal slab (Fig. 18). If the mode has $\mathrm{C}_{4 v}$ symmetry inherited by the square lattice, its field structure becomes not compatible with $\mathrm{C}_{2}$ symmetric free-space waves, and as such, coupling with the radiation channel becomes negligible, ideally zero. This kind of BIC is termed symmetry-protected. As such, a BIC can be considered as a resonant mode with an infinite radiative $Q$-factor in an open cavity, a non-Hermitian system in which energy would remain localized, ideally indefinitely, unless other loss mechanisms such as unavoidable material absorption and defects are taken into account. It is clear from this simple view that the build up factor of the optical field associated with a diverging $Q$-factor, practically limited also in numerical simulations to $\sim 10^{10}-10^{13}$, challenges in principle all highly performing resonators, from high finesse electromagnetic cavities and photonic crystal defect nanocavities to whispering gallery mode resonators (for what concerns dielectric materials). Thus, many processes and phenomena, below discussed, may be enhanced in BIC-based systems.

A BIC point can also be seen as a specific point in the parameter space (radius, height, refractive index, incidence angle) at which outgoing radiation components may cancel out each other due to perfect destructive interference in the free space region. This mechanism due to a precise balancing is associated with the so-called accidental BICs and is generally related to a fine tuning of a geometric parameter of the structure that occurs not only in highly symmetric points but at any angle of incidence [319]. The onset of accidental BICs as modal interference between two distinct resonance modes of the same resonator is also called Friederich-Wintgen BIC [349]. In this case, strong coupling among two modes belonging to close dispersion bands, at a given momentum value (angle of incidence), generates an avoided crossing in which one of the bands becomes vanishing with infinite radiative $Q$-factor despite being above the light line [356]. Similarly, Fabry-Pérot BICs can be obtained by interference between 
(a)

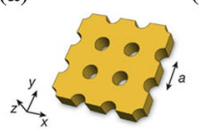

(b)

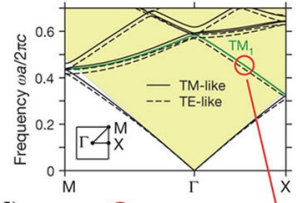

(c) $10^{4} 10^{6} 10^{8}$ to $\infty$

(d) ${ }_{10^{8}}$

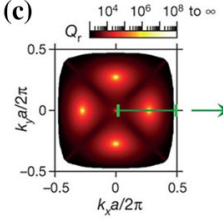

(h)

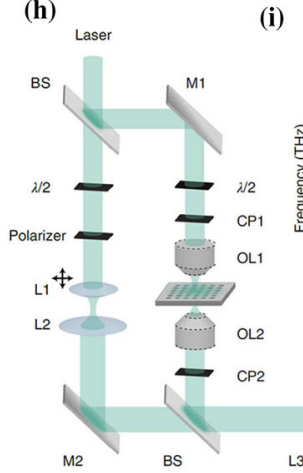

(e) (e)
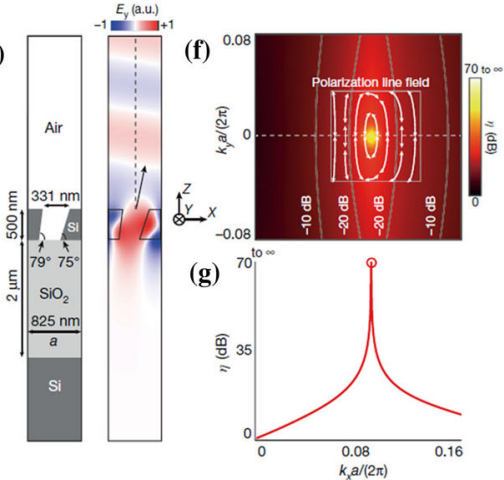

(I)

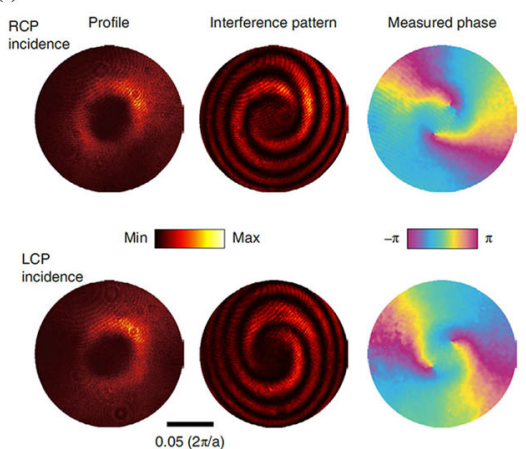

Fig. 18 a Photonic crystal slab with square symmetry. b Numerically calculated dispersion band diagram for transverse electric (TE) and transverse magnetic (TM) modes associated to a. c Corresponding radiative $Q_{r}$-factor mapped in the reciprocal space and detailed (d) diverging $Q_{r}$-factor of accidental BIC with related profile of the confined (major) component $E_{z}\left(\mathrm{TM}_{1}\right)$. e Topologically unidirectional BIC with slanted structure with related vortex polarization profile (f) and top-down asymmetry emission loss $\eta$ (g). h Layout of the experimental setup for spin-to-orbital angular momentum conversion analysis of a photonic crystal slab with square symmetry at the BIC point shown in the dispersion diagram in i. Resulting interference profile demonstrating generation of orbital angular momentum with opposite sign upon spin flip of the input right-circularly polarized (RCP) or left-circularly polarized (LCP) beam. a-d Adapted with permission from [319]. e-g Adapted with permission from [343]. h-l Adapted with permission from [345]

two resonators [316]. Other formation mechanisms pertain to reverse engineering with artificial potentials [357].

In all these circumstances, when inspecting the vector field structure of the BIC mode, it is possible to recognize a vortex structure [331,344]. This property is intimately related to the general nature of BICs. Indeed, even when compatible with radiation in terms of geometric transformation (the case of accidental BIC in non symmetric points), the BIC is a polarization singularity in the reciprocal space $[320,358]$. BIC points are pinned at a point $\overrightarrow{\boldsymbol{k}}_{B}$ that defines the core of a polarization vortex of free-space waves, which makes polarization undefined (Fig. 18e-g). This makes BICs topologically incompatible with free space solutions of Maxwell's equations that have a well-defined polarization representation. A direct consequence is the possibility of polarization engineering of the radiation pattern in structured light [333,359], also 
carrying orbital angular momentum, in proximity of BICs (Fig. 18h-1). The BIC is indeed associated with a topological charge, which can merge or annihilate with other BICs [320], and whose sign and strength determine the nature of the field structure and its orbital angular momentum. Radiation engineering with unidirectional propagation of light has also been demonstrated as a result of topological charge bouncing [343] (Fig. 18e-g). In addition, a partial conversion from spin degree of freedom of input light to orbital angular momentum has been recently observed, despite the homogeneity of the dielectric structure, just thanks to the inhomogeneous orientational response around a BIC [345] (Fig. 18h-1).

Another straightforward way of visualizing BIC formation concerns multipolar radiation engineering [360]. A complex radiation source can be decomposed as a multipole series of elementary electric and magnetic radiation components. Their suitable combination allows not only determining unconventional coupling with waveguides like for Janus and Huyghens dipoles [361], but also describing the radiation cancellation occurring in all non-radiating sources such as BICs. In Ref. [360], this powerful technique is applied to explain the origin of both symmetry-protected and accidental BICs as cancellation in the direction of a given wavevector of all vector harmonics composing the optical resonant field. This mechanism can be directly translated to increase coupling efficiency by engineering the incident source to excite the main multipole components.

While the theoretical predictions of these exotic optical modes were done before the 2000s with different names as trapped modes, embedded eigenvalues, dark states, the first systematic experimental evidence appeared later. In 2008, Marinica et al. [350] showed that the interaction between trapped electromagnetic modes in two parallel dielectric gratings and two arrays of thin parallel dielectric cylinders can lead to scattering resonances with practically zero width. Plotnik et al. [317] showed the coupling of BICs in an optical waveguide array structure, demonstrating that the symmetry breaking of the system couples these special states. Further progress has been done later on photonic crystal slabs supporting BICs [319]. The key point in observing BICs in this kind of structures is that in practical structures several circumstances turn these ideal states to real radiating modes, also termed quasi-BICs, whose spectral profile has a Fano shape as produced by partial coupling with the continuum. The Fano resonances then collapse ideally as a result of zero and pole merging in a pure BIC, which is not excitable from the far field, but requires near field coupling. Experimentally, the $Q$-factor of quasi-BICs is limited by material absorption, scattering loss due to inhomogeneous dielectric/geometric features and Bloch's wavevector spreading due to finite sizes of the fabricated structure [328]. Differently from the symmetry-protected BICs, whose $Q$-factor dramatically decreases far from the $\Gamma$-point, for accidental BICs, $Q$-factor appeared to decay more slowly, remaining high against input beam collimation [331,339].

To stress the relevance of BIC concept for having high- $Q$ resonances, it must be noted that the surviving $Q$-factor is still much larger than leaky resonances for which no mechanisms prevent radiation loss. Typical $Q$-factor of $10^{5}$ can be achieved in high index materials $[319,362]$ with modes that are delocalized in the transverse plane and confined only axially, which allows many applications, from lasing [333,363] to non-linear frequency conversion $[336,364]$ and ultrasensitive sensing and imaging 
$[327,330,365]$. The delocalized nature of BICs, provided by their collective resonance behavior and topological properties, makes the existence of the mode robust against external local perturbations and defects. The large area nature of BICs is a first key factor in determining their success for applications, but also seemingly a limitation in some cases. Recently, this limitation was overcome by the demonstration of quasi-BIC modes of large $Q$-factor even in isolated sub-wavelength dielectric particles [364,366368].

We will now introduce, without aiming to be exhaustive, some important advances regarding applications of BICs. First, a general overview of enhanced light-matter interaction based on BICs will be summarized; then, more attention will be devoted to sensing applications.

\subsection{Enhanced light-matter interaction with BICs}

Nonradiating states of light induce strongly localized EM fields, similar to their plasmonic counterparts, in all-dielectric nanomaterials with negligible or very limited losses. As such, they promise of extending the results of plasmonics to the visible and UV range, where dielectric absorption is in general limited. In addition, due to the multipolar nature and interferences associated with such states, all-dielectric platforms allow several novel effects not even possible in plasmonics, the latter being mainly driven by the physics of electric hot spots and electric dipole modes. As mentioned above, large local near-field enhancement due to the excitation of BIC modes in photonic crystal slabs or metasurfaces can be obtained. Careful control of some parameters, such as slab thickness [370], holes or pillars diameter [363], cladding refractive index [330] or other geometric features, allow the optimization of the resonance mechanism. Numerical analysis showed that such radiationless modes, which for reciprocity would not be excitable, can instead be excited with planar incident waves because of finite precision in frequency tuning [344,358]. As a consequence, a giant field enhancement distributed over the whole structure of about $10^{6}$ was numerically calculated for a specific design in Ref. [370]. To have access to this local field confinement is a crucial point. To facilitate light coupling while providing a tunability degree of freedom, an asymmetry parameter can be introduced in the unit cell to break the geometry symmetry and induce the quasi-BIC regime [371].

An important impulse to the research in this field was given by Kodigala et al. [331] that demonstrated a highly efficient lasing action based on accidental BICs in an InGaAsP nanostructure. Directional lasing and wavelength control with temperature was later shown in GaAs nanocylinders [332]. While early surface emitting vertical lasers based on photonic crystals are now recognized as associated with symmetry protected BICs, Kodigala et al. moved a step forward exploiting a precise engineering of the dispersion curves of the photonic structures by tuning the radius parameter of the pillars in a InGaAsP patterned membrane [331]. In such a way, an accidental BIC at normal incidence was achieved, resulting in a high- $Q$ factor over a rather large solid angle away from normal incidence. This mechanism favored input coupling and light extraction efficiency. Remarkably, lasing action was still preserved, reducing the finite extent of the photonic crystal to smaller sub-domains of a few pillars. The laser mode 


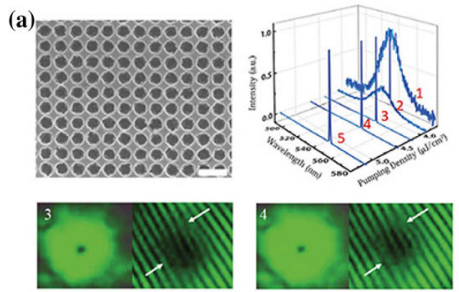

(c)

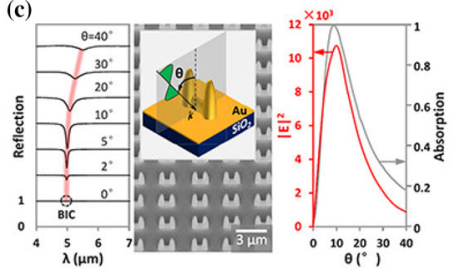

(e)

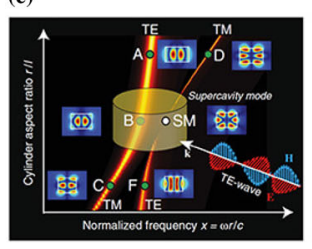

(b)

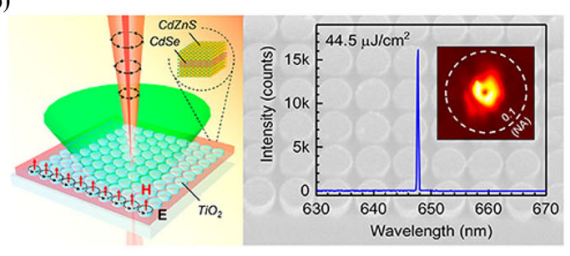

(d)

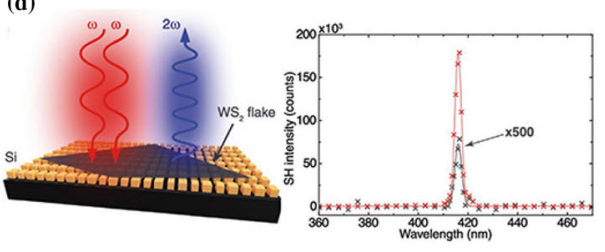

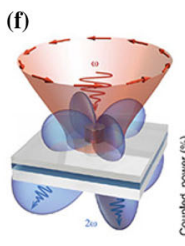
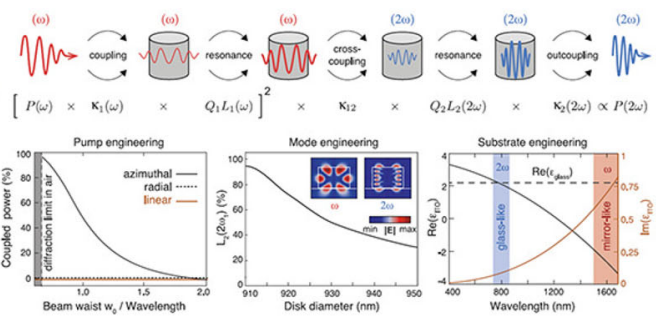

Fig. 19 a Perovskyte photonic crystal slab with square symmetry in which vortex-structured lasing action originated from symmetry-protected BIC. b Lasing action produced by a film of colloidal nanoplatelets with square symmetric nanocylinders of titanium dioxide at a BIC. $\mathbf{c}$ Hybrid dielectric/plasmonic structure capable of near-field enhancement in correspondence of perfect absorption condition with suitable detuning from a BIC. d Enhanced second harmonic generation from a transition metal dichalcogenide flake with a inplane symmetry broken metasurface tailored to support a quasi-BIC. e Representation of modal interference within a single dielectric scatterer producing a super-cavity mode or quasi-BIC.f Enhanced second harmonic generation by a single nanoparticle supporting a quasi-BIC with optimal coupling efficiency via azimuthally polarized input beam, and diagram of doubly resonant mechanism at both fundamental and harmonic frequencies. a Adapted with permission from [333]. b Adapted with permission from [334]. c Adapted with permission from [369]. d Adapted with permission from [336]. e Adapted with permission from [366]. f Adapted with permission from [364]

can be endowed with orbital angular momentum since structured vector beams radiate from BICs, as indeed demonstrated in ultrafast-addressable microresonator of perovskytes generating vortex beams [333] (Fig. 19a). A film of colloidal CdSe/CdZnS core-shell nanoplatelets with square arrays of nanocylinders made of titanium dioxide was used in ref. [334] to achieve optically pumped room temperature lasing at visible wavelengths (Fig. 19b). This is a characteristic of a certain relevance since it opens new scenarios in the direct manipulation of the quantum freedom degrees of light. Furthermore, a recent study on symmetry-protected BICs suggested the possibility of releasing the inhomogeneity and anisotropy of the material necessary to give rise to spin-to-orbital angular momentum conversion [372]. In case of BICs, indeed, the intrinsic dispersion inhomogeneity/anisotropy mediated spin-to-orbit coupling [345] (Fig. 181). In addition, spin-polarized directive coupling associated with 
the pseudo-spin of Poynting vector vortices of degenerate BIC modes excited by circular polarizations was recently shown [344]. This was ascribed to a manifestation of the quantum spin-Hall effect of light generated by the transverse spin angular momentum of the tightly confined field at the BIC.

Besides lasing effect, surface-enhanced fluorescence in all dielectric photonic crystals along with enhanced spontaneous Raman scattering were discussed [339]. The authors also described a hybrid system of isolated plasmonic nanoparticles coupled to the near-field of the BIC. They showed the feasibility to enhance surface enhanced Raman scattering, which may be extended to many other plasmonic phenomena, provided that the perturbation to the BIC mode is properly taken into account.

Significant advances in the exploitation of quasi-BIC near-field enhancement were recently established in several papers. The idea is that an interplay between high but finite radiative $Q$-factor, balanced by the nonradiative $Q$-factor, must be taken into account to maximize the near field enhancement in real-world absorbing structures. This condition is achieved, as first recognized for special Fano resonances [373] and erlier in plasmonic effective mode volume picture [374], at the critical coupling. Feasible near field enhancement up to $10^{4}$ in quasi-BIC regime under critical coupling is claimed even in hybrid dielectric-plasmonic nanostructures, and found correspondent to perfect absorption condition [369] (Fig. 19c). In this direction, so-called virtual critical coupling theory was recently presented with the aim to overcome the poor external coupling efficiency of high- $Q$ resonators in quasi-BIC regime without relying on loss by using a temporal modulated excitation [375].

BIC physics was soon applied in nonlinear photonics where wave mixing may be facilitated by large optical fields. BICs were applied for second [335,336] and third $[337,338]$ harmonics generation with surprising efficiency, with enhancement of $10^{3}$ with respect to unpatterned regions with silicon or gallium phosphide nanostructures tailored for quasi-BIC maximum efficiency, either with large-area resonators [335338] by using the intrinsic material nonlinearity for third harmonic generation or in hybrid systems with materials like transition metal dichalcogenides (Fig. 19d) coupled to the resonator for second harmonic generation, or single-particle resonance tailoring [364,366] (Fig. 19e). Matching the polarization pattern of the mode by using an azimuthally polarized input beam was shown to increase dramatically the coupling efficiency for second harmonic conversion [364,376] (Fig. 19f). Recently, multiwavelength and multistep BIC enhanced processes were discussed [377], and high frequency conversion up to 11th harmonic in the UV range was proposed with femtosecond excitation [378].

\subsection{Optical sensing with BICs}

The possibility of engineering high quality factor cavities in microsphere resonators, ring resonators, photonic crystal resonators, waveguides and circuits has led to interesting applications for perturbation-type measurements used in ultrasensitive (single molecule) biosensing. In this direction, also photonic crystal slabs and metasurfaces supporting BICs were investigated for deployment as biological and chemical sensors $[327,330,365,379]$. Indeed, since the light confined in the photonic nanostructure at the 
BIC has a significant fraction of power in the evanescent tail, the peak wavelength of the resonance is strongly affected by the refractive index (RI) of the external medium or by molecular adsorption in case of surface binding events. This allowed to realize BIC-based sensors with high sensitivity to environment media variation. The strongly confined field over large-area allowed the direct detection of a monolayer of ultralowmolecular-weight molecules with transparent silicon nitride in the visible range [365] and atomically thin graphene layers with in-plane asymmetric silicon metasurfaces in the IR range [327]. The linewidth of a resonance-based device affects its figure of merit (FOM), defined as the ratio between the sensitivity and the full width at half maximum (FWHM), which ultimately represents the capability to follow tiny changes in the environment RI, thus providing sensors with a low limit of detection. In Ref. [379,380], FOM values as large as 450 were reported applying BICs also for ultrasensitive bio-molecular detection. A capability of detecting antigen/antibody bindings with superficial density as low as 0.2 molecules $/ \mu \mathrm{m}^{2}$ was reported in Ref. [365]. By addressing the physical transduction mechanism provided by the evanescent field, the potential tuning of the sensitivity was explored in ref. [381,382] with potential sensitivities as large as $4000 \mathrm{~nm} / \mathrm{RIU}$.

Typical sensing schemes and setups based on BICs made use of normal incidence launching platforms to minimize the optical interrogation setup. BIC-based sensors appear also scalable in all spectral ranges with no specific limitations. Thus, such devices may have great potential for the realization of low-cost sensing platforms, possibly integrated in microfluidics and capable of operating with minimal sample volumes and detecting analytes at extremely low concentration levels. Sophisticated readout systems based on BICs were recently presented [326,327,330]. Indeed, the standard sensing mechanisms can be combined with advanced imaging-based optical data acquisition and processing methods to build a superior ultrasensitive label-free analytical platform for biosensing. Yesilkoy et al. [327] showed a novel technique able to acquire spatially resolved spectra from millions of image pixels and extract highthroughput digital sensing information at the level of less than three molecules per $\mu \mathrm{m}^{2}$, using smart data-processing tools. The device enabled the spectral data retrieval without using spectrometers, useful for portable diagnostic applications. Romano et al. [330], instead, demonstrated the combination of surface-enhanced fluorescence and resonant shift sensing during microscopic raster scanning of cells with micrometric resolution. To increase refractometric sensitivity, a dual BIC-based mechanism was adopted for enhancing the fluorescence emission of a dye in correspondence of an accidental BIC close to normal incidence at critical coupling. The fluorescence emission was a function of the spatially variant dielectric environment in the near-field of the structure. Taking advantage of a concurrent refractometric sensing scheme based on Fano interference in the fluorescence emission, the device was used to map the spatially variant cell environment, with femto-liter volume sampling and enhanced refractometric resolution of $10^{-5}$ RIU. A parallel with another photonic platform for sensing may allow us to better highlight some peculiar aspects of BIC sensing. In particular, typical photonic crystal nanocavities used for refractometric sensing had experimental spectroscopic sensitivities of the order $10^{2} \mathrm{~nm} / \mathrm{RIU}$ [383], whereas in more complex designs like slotted cavities [384], a spectroscopic sensitivity as large as $1500 \mathrm{~nm} / \mathrm{RIU}$ was reported. Experimental resolution in nanocavities was at the best on 
the order of $10^{-5}$ RIU [383], but all used typically materials non transparent in the visible range of light (i.e. mainly silicon) with sensing zones confined to nanoscale defect points. In contrast, BIC sensing mechanism did not require a nanocavity localized in a specific point or line defect given the nature of the confinement. Since the modes were delocalized in the transverse plane, they were used for space variant mapping with RI resolution of the order of $10^{-5}$ RIU like best nanocavities. In addition, the advantage of large-area sensing is also complemented by the facile launching scheme at normal incidence with a far field source. Indeed, the consequent versatility and facility of application challenges most of high- $Q$ resonating systems, and of course in first place guided resonances in photonic crystal slabs.

The other frontier of BIC-based sensing relies on IR absorption, cleverly proposed to take advantage of high- $Q$ resonances based on quasi-BICs [326]. Germaniumbased metasurfaces were realized for detecting the molecular absorption fingerprints over a broad range of IR wavelengths by adopting a bar-coding approach. Operating at the quasi-BIC regime by engineering a suitable in-plane symmetry breaking, the absorption spectrum of the specimen was sampled with sharp multiplexed resonances in a pixelated chip in which each pixel was associated with a slightly different quasiBIC resonance in order to cover a certain spectral range [326]. A further advance was discussed in ref. [385], in which the multiplexing approach associated to different pixel geometry supporting sampling resonances was substituted with a single nanostructure with monotonically increasing dispersion band in order to control the spectral positions of the resonances with the incidence angle. In such a way, a single metasurface was necessary by performing angle-dependent reflectance measurements to acquire the full molecular absorption spectral fingerprint of several specimens.

\section{Optical anapoles}

The physics of interference and interplay between different kinds of multipolar modes underlies nanoscale light manipulation, providing information about the basic characteristics of matter, including absorption, dispersion, and optical activity. Generally, electromagnetic properties of matter can be described by multipoles expansion, i.e. a combination of magnetic and electric dipoles commonly represented by oscillating charges and loop currents, respectively [386]. In 1967, Dubovik [387], in the general framework of research into plasma stability in Tokamaks, introduced a new class of moments being excluded from the family of electric and magnetic moments, with different time-space symmetries but appearing in similar orders in the expansion set with respect to magnetic moments, termed toroidal moments. The toroidal dipole corresponds to currents flowing on the surface of a torus. In the far-field zone, they produce the same radiation patterns as corresponding electric or magnetic multipoles. In particular, when an electric dipole and a toroidal dipole are coexcited and spatially overlapped with the same radiation magnitude but out of phase, they cancel the scattering of each other in the far field region, thus the scatterer becomes invisible. The resulting nonradiating source is known as anapole (Fig. 20a) [388,389]. An overview of recent developments about anapoles and their applications, together with in-depth analytical analysis, can be found in dedicated reviews [388,390]. As for BICs, and in 
particular quasi-BICs in single scatterers [364,366,367] where interference between modes is responsible for radiation suppression, the radiationless nature of the modal engineering underlying the anapole condition is accompanied by large local optical field boosting light-matter interaction, from which a plethora of processes and phenomena can be enhanced. Herein, after introducing anapoles, a selection of relevant applications will be discussed.

Anapole concept was introduced in 1957 by Yakov Zel'dovich in the physics of elementary particles to explain the parity violation of weak interaction in atomic nuclei [391] and experimentally demonstrated by Wood et al. in 1997 [392]. More recently, the anapole moment was suggested as the only allowed electromagnetic moment in Majorana fermions to describe the origin and composition of dark matter in the universe [393,394].

The experimental observation of anapole modes in optics has become possible only with advances in nanofabrication and metamaterials, enabling the discussion of such phenomena in a wide range of the electromagnetic spectrum, from microwave to nearinfrared frequencies [395-398]. Optical anapoles were experimentally demonstrated for the first time at the microwave in a toroidal metamaterial consisting of a metal screen with a dumbbell-shaped aperture as structural element (Fig. 20b) [399]. This class of metamaterials enables a novel mechanism of resonant electromagnetic transparency, producing very narrow and characteristic symmetric Lorentzian transparency lines with $Q$-factors exceeding 300. Similar planar plasmonic metamaterials, consisting of a multilayered structure containing a planar array of vertical split-ring resonators suspended in a dielectric medium and covered with a perforated gold film, was shown to support toroidal dipoles and anapole excitations in the optical part of the spectrum (Fig. 20c) [400].

Despite such artificially structured media on the subwavelength scale may provide the opportunity to explore exotic optical phenomena, the anapole response can be damped by Joule losses. Thus, significant attention has been directed to the study of all-dielectric structures [401]. Under proper excitation, the anapole state can be generated even in simple isolated scatterers, such as dielectric spheres, disks and nanorods [402-404]. Specifically, nearly pure anapole modes were excited in suitably tuned nanodisks capable of exhibiting leading contribution of solely electric and toroidal dipoles, being all other modes strongly suppressed. In particular, Miroshnichenko et al. [402] showed that a silicon nanodisk of height $50 \mathrm{~nm}$, by tuning the diameter from 200 to $400 \mathrm{~nm}$, may support the anapole mode, which was experimentally excited and observed via scanning near field optical microscopy. A spectral dip in the far-field scattering corresponding to the dark anapole was found in the visible, at $550 \mathrm{~nm}$; as the diameter increased, the dip red-shifted and became more pronounced (Fig. 20d, e). This was the first experimental demonstration of an anapole in the visible wavelength range. Wei et al. [405] demonstrated that it is possible to excite the anapole mode even in an isotropic high-index dielectric nanosphere of radius $100 \mathrm{~nm}$ placed at the focal point of two counter-propagating focused radially polarized beams under $4 \pi$ configuration. In addition, excitation of anapole modes in silicon nanoparticles was found facilitated, again using structured light illumination, with azimuthally polarized focused beams, which enabled exciting ideal anapole modes of magnetic type in dielectric nanoparticles [406]. 
(a)

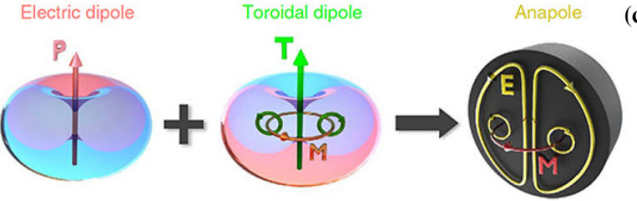

(c)

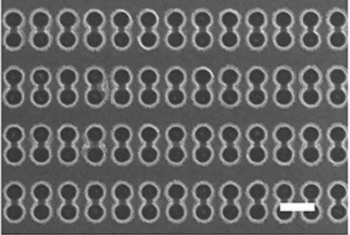

(b)
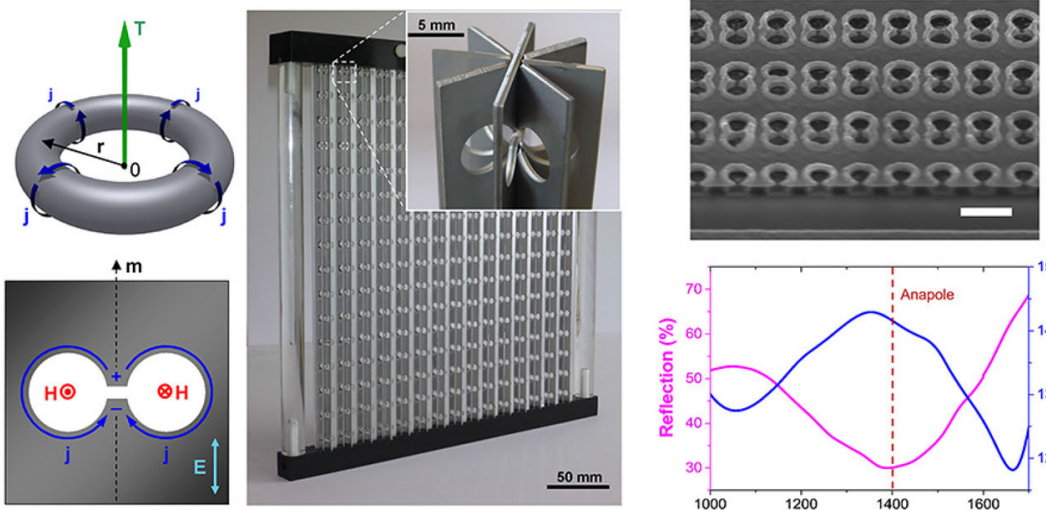

$\Delta \mathrm{m}$
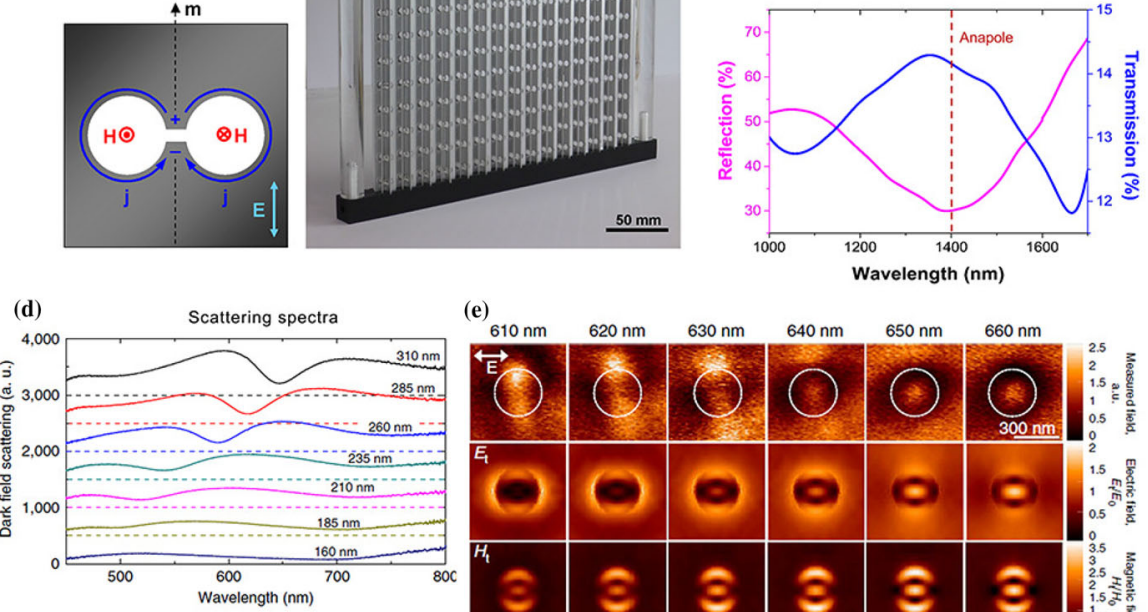

(e)

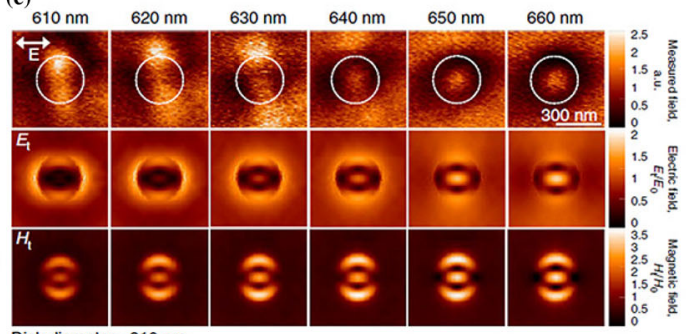

Disk diameter - $310 \mathrm{~nm}$

Fig. 20 a Schematic representation of an anapole state as a superposition of electric dipole and toroidal dipole modes. Adapted with permission from [402]. b First experimental observation of dynamic anapole states in microwave metamaterials. Poloidal currents flowing on a surface of a torus along its meridians create toroidal dipole moment $\mathrm{T}$ and induced charge current distributions around a dumbbell-shaped aperture (left). A photograph of the designed stainless steel slab (right). Adapted with permission from [399].c An optical anapole metamaterial consisting of a planar array of vertical split-ring resonators suspended in a dielectric medium and covered with a perforated gold film. Adapted with permission from [400]. d Experimental dark field scattering spectra of silicon nanodisks with a height of 50nm and a diameter ranging from 160 to 310 $\mathrm{nm}$. e Near-field enhancement around the silicon nanodisk with diameter of $310 \mathrm{~nm}$; top row, experimental NSOM measurements, middle and bottom rows calculated transversal electric and magnetic near-field, respectively. White dashed lines indicate the disk position. Adapted with permission from [402]

\subsection{Anapole applications}

As mentioned above, the concept of anapole involves important implications in nanophotonics. Indeed, it provides an attractive way to efficiently control light-matter interaction at nanoscale, that can be exploited in cloaking, enhanced spectroscopies, sensing and nonlinear optics, as well as in advanced devices, including nanolasers 
(a)

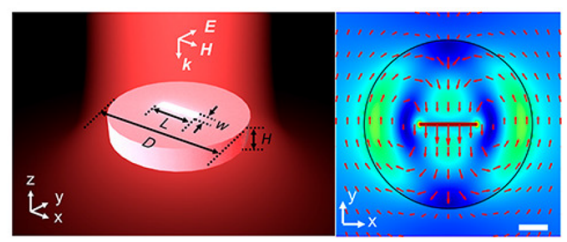

(b)

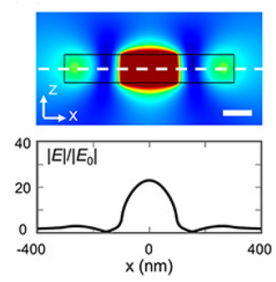

(c)

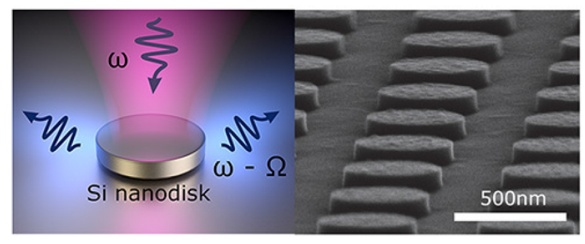

(d)

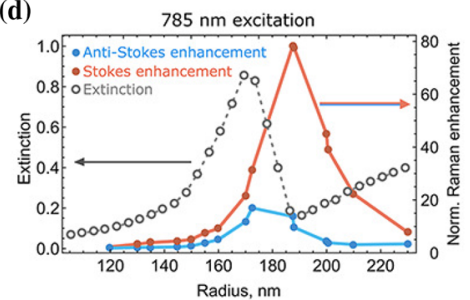

Fig. 21 a Slotted nanodisk sustaining anapole mode for enhanced light confinement. Near-field profiles on the xy plane in the middle of the disk and $\mathbf{b}$ near-field enhancement of the electric field in the nanodisk structure. Adapted with permission from [410]. c Anapole-enhanced Raman scattering in a silicon nanodisk, scheme and SEM image of the disk array. d Integrated Stokes and anti-Stokes intensities of the $522 \mathrm{~cm}^{-1}$ Si phonon line for $785 \mathrm{~nm}$ excitation as a function of disk diameter (solid curves) and measured extinction values at $785 \mathrm{~nm}$ (dashed curve). Adapted with permission from [416]

[407-409]. Thefore, the realization of nonradiating states of light in all-dielectric nanostructures expands the results already carried out by plasmonics.

A strong field enhancement factor can be achieved by utilizing optical anapole modes since electric and toroidal dipoles cancel each other far away from the structure, while concentrating the optical field inside the resonator. Yang et al. [410] demonstrated a light intensity enhancement exceeding three orders of magnitude in a $\mathrm{Si}$ all-dielectric nanodisk, exploiting anapole generation. Cleverly, a further boost of the electric field enhancement was introduced by a slot area with high-contrast interfaces in order to get access to the strongest electric hotspot sitting inside the particle (see Fig. $21 \mathrm{a}, \mathrm{b})$.

To further increase the field enhancement factor, several methods were proposed in hybrid dielectric/plasmonic structures [411-414]. The interaction of anapole and plasmons in a metal-dielectric structure was explored in [415]], where a side-by-side gold nanostrip dimer plasmon resonator was padded on the long sides of the air slot in a silicon Mie resonator nanodisk. A dark plasmon mode was excited by illumination of linearly polarized light via the anapole-plasmon coupling. Such interplay generated hybrid modes showing anticrossing behavior with a Rabi split of $120 \mathrm{meV}$, matching the strong-coupling criterion between anapole and plasmon. This strong coupling produced intensity enhancements at the two peaks of the hybrid mode of 4 and 20 times their respective uncoupled cases. In addition, the anapole-assisted dark plasmon excitation simplified the experimental implementation by eliminating the need to manipulate the light field in free space.

Field enhancements corresponding to the anapole condition can improve efficiency in linear and nonlinear interactions at the nanoscale. This was the case of the Raman 
response of Si nanoparticles, as demonstrated both theoretically and experimentally [416]. Compared to an unstructured Si film, the authors reported a 2 orders of magnitude enhancement of the Si Raman scattering intensity per unit volume for dielectric nanodisks excited at the same wavelength of the anapole state (see Fig. 21c, d). Stokes and anti-Stokes Raman spectra from arrays of Si disks were collected using $785 \mathrm{~nm}$ excitation. For the case of a disk radius around $190 \mathrm{~nm}$, this structure supported the anapole state at the excitation wavelength of $785 \mathrm{~nm}$, as revealed by the minimum in extinction/reflectivity. Matching the pump laser wavelength, an enhancement of the Stokes emission of a factor of $\sim 80$ compared to an unstructured Si film was achieved.

Further works clearly demonstrated a correlation between enhancement of nonlinear optical processes with the near-field enhancement in systems sustaining anapole states. Grinblat et al. [417] demonstrated that an all-dielectric germanium nanodisk supporting anapole states exhibits a strong third order nonlinear response and efficient third harmonic generation in the optical regime. Specifically, by pumping the nanosystem close to the anapole mode, they found that the response of the third harmonic signal versus fundamental wavelength matched that of the cube of the simulated inner electric energy. They reported a conversion enhancement of 4 orders of magnitude with respect to the unstructured germanium film. A similar nonlinear optical response was observed also in the case of degenerate four-wave mixing where two different pump wavelengths were coupled to a single high-order resonant mode [418].

Recently, interference phenomena generating anapole modes in high-refractive index nanoparticles arranged in planar metasurfaces were exploited for sensing [419]. In particular, nanostructured metasurfaces of periodic arrays of nanocuboids on a glass substrate supporting anapole mode were properly designed such that a resonance with ultrahigh $Q$-factor above one million could be excited at the target infrared wavelength of $1.55 \mu \mathrm{m}$. The spectral position of the anapole-induced resonance depended on the refractive index of the cladding over the resonant structure exhibiting a sensitivity up to $180 \mathrm{~nm} / \mathrm{RIU}$. Such values, combined with the ultrahigh $Q$-factor, allowed for refractometric sensing with very low limits of dection in a broad range of refractive indices [419]. A different approach for biodetection exploiting anapole modes was proposed in [420]. The authors simulated the behavior of a silicon nanodisk incorporating a nanoslot at the disk center fabricated over a dielectric thin film of defined thickness on top of a metal back-reflector. The nanoslot was designed to be approximately the same size as a single biomolecule. This configuration made accessible the location of the electric field node of the anapole mode and took advantage of the hybrid coupling effect to achieve even greater field enhancements. The strictly confined electromagnetic field produced a theoretical single point electromagnetic hotspot with up to $6.5 \times$ enhancement of electric field, in 630-650 nm wavelength range, and increased up to $25 \times$ by coupling additional electromagnetic energy from the underlying metallic substrate. Due to the fluorescence amplification of fluorophores captured within the hotspot, the authors envisaged that biomolecular events occurring there could be observed with high signal-to-noise ratio by monitoring the emission of single fluorophores associated with single molecules. 


\section{Conclusions}

The ability to manipulate light at the nanoscale is not a prerogative of the humankind. Millions of years of evolution provided several species of animals, plants, flora and protists with more or less ordered nanostructures able to selectively reflect and transmit light or to handle it in more complex ways, mainly for camouflage, intra- and inter-species communication or optimization of sunlight exploitation. The intricate architectures at the basis of these properties may represent a source of inspiration in the realization of novel photonic devices.

Modern fabrication techniques and the discovery of new artificial materials, with unique properties not found in nature (i.e., metamaterials), have been allowing to cross the limits of natural optical phenomena, mainly through the introduction of plasmonics and dielectric nanophotonics. These new nanotechnologies seem to meet the rapidly increasing demand of high-performance and low-cost integrated optical devices and systems. Optical metasurfaces - due to their subwavelength thickness, thin and planar form factor, and the possibility of being batch fabricated at potentially low cost using standard micro/nanoelectronic techniques_-are offering an alternative approach to the realization of new optical components. Furthermore, metasurfaces not only reproduce but also introduce new functionalities compared to those obtainable with conventional photonics.

This paper is an attempt to contribute at the comprehension of what is going on in this emerging and rapidly moving research field. It moved from a survey of natural photonic structures found in living species, whose study is recently revealing the important role they play in light manipulation. Then, after a short recall of the concepts at the basis of the light-matter interaction in metallic single and arrayed nanostructures, some of the cutting edge plasmonics configurations and applications are reported. A final section was dedicated to the rising possibilities offered by the technology of dielectric-based metasurfaces, in particular, lingering on the new concepts of bound states in continuum and anapoles, in perspective capable of overcoming the intrinsic limits of plasmonics, and so paving the way to new unexplored approaches in light manipulation at the nanoscale.

Open Access This article is licensed under a Creative Commons Attribution 4.0 International License, which permits use, sharing, adaptation, distribution and reproduction in any medium or format, as long as you give appropriate credit to the original author(s) and the source, provide a link to the Creative Commons licence, and indicate if changes were made. The images or other third party material in this article are included in the article's Creative Commons licence, unless indicated otherwise in a credit line to the material. If material is not included in the article's Creative Commons licence and your intended use is not permitted by statutory regulation or exceeds the permitted use, you will need to obtain permission directly from the copyright holder. To view a copy of this licence, visit http://creativecommons.org/licenses/by/4.0/.

\section{References}

1. A. Karabchevsky, A. Katiyi, A.S. Ang, A. Hazan, Nanophotonics 9, 3733 (2020)

2. I. Utke, S. Moshkalev, P. Russell, Nanofabrication Using Focused Ion and Electron Beams: Principles and Applications (Oxford University Press, Oxford, 2012) 
3. F. De Angelis, M. Malerba, M. Patrini, E. Miele, G. Das, A. Toma, R.P. Zaccaria, E. Di Fabrizio, Nano Lett. 13, 3553 (2013)

4. R. Meade, J.N. Winn, J. Joannopoulos, Photonic crystals: Molding the flow of light (1995)

5. D. Threm, Y. Nazirizadeh, M. Gerken, J. Biophotonics 5, 601 (2012)

6. S. Romano, G. Zito, S. Torino, G. Calafiore, E. Penzo, G. Coppola, S. Cabrini, I. Rendina, V. Mocella, Photonics Res. 6, 726 (2018)

7. S.A. Maier, Plasmonics: Fundamentals and Applications (Springer, New York, 2007)

8. J.A. Schuller, E.S. Barnard, W. Cai, Y.C. Jun, J.S. White, M.L. Brongersma, Nat. Mater. 9, 193 (2010)

9. V. Greanya, Bioinspired Photonics: Optical Structures and Systems Inspired by Nature (CRC Press, Boca Raton, 2015)

10. F.G. Omenetto, Photonics in nature-opportunities and challenges at the biological interface (2019)

11. C.M. Soukoulis, M. Wegener, Nat. Photonics 5, 523 (2011)

12. N.I. Zheludev, Y.S. Kivshar, Nat. Mater. 11, 917 (2012)

13. J. Pendry, Y. Luo, R. Zhao, Science 348, 521 (2015)

14. W. Gao, M. Lawrence, B. Yang, F. Liu, F. Fang, B. Béri, J. Li, S. Zhang, Phys. Rev. Lett. 114, 037402 (2015)

15. F. Monticone, A. Alu, Rep. Prog. Phys. 80, 036401 (2017)

16. S.M. Choudhury, D. Wang, K. Chaudhuri, C. DeVault, A.V. Kildishev, A. Boltasseva, V.M. Shalaev, Nanophotonics 7, 959 (2018)

17. D.G. Baranov, D.A. Zuev, S.I. Lepeshov, O.V. Kotov, A.E. Krasnok, A.B. Evlyukhin, B.N. Chichkov, Optica 4, 814 (2017)

18. X. Chen, L. Huang, H. Mühlenbernd, G. Li, B. Bai, Q. Tan, G. Jin, C.-W. Qiu, S. Zhang, T. Zentgraf, Nat. Commun. 3, 1 (2012)

19. X. Ni, A.V. Kildishev, V.M. Shalaev, Nat. Commun. 4, 1 (2013)

20. G. Li, M. Kang, S. Chen, S. Zhang, E.Y.-B. Pun, K.W. Cheah, J. Li, Nano Lett. 13, 4148 (2013)

21. X. Yin, Z. Ye, J. Rho, Y. Wang, X. Zhang, Science 339, 1405 (2013)

22. A. Parker, Natural photonic structures: an overview, in Optical Biomimetics (Elsevier, Amsterdam, 2012), pp. 1-20e

23. M. Kolle, Photonic Structures Inspired by Nature (Springer, New York, 2011)

24. I.C. Cuthill, W.L. Allen, K. Arbuckle, B. Caspers, G. Chaplin, M.E. Hauber, G.E. Hill, N.G. Jablonski, C.D. Jiggins, A. Kelber et al., Science 357, eaan0221 (2017)

25. M. Jacobs, M. Lopez-Garcia, O.-P. Phrathep, T. Lawson, R. Oulton, H.M. Whitney, Nat. Plants 2, 1 (2016)

26. E. Moyroud, T. Wenzel, R. Middleton, P.J. Rudall, H. Banks, A. Reed, G. Mellers, P. Killoran, M.M. Westwood, U. Steiner et al., Nature 550, 469 (2017)

27. P. Vukusic, B. Hallam, J. Noyes, Science 315, 348 (2007)

28. M. Burresi, L. Cortese, L. Pattelli, M. Kolle, P. Vukusic, D.S. Wiersma, U. Steiner, S. Vignolini, Sci. Rep. 4, 6075 (2014)

29. K. Kertész, G. Molnár, Z. Vértesy, A. Koós, Z. Horváth, G. Márk, L. Tapasztó, Z. Bálint, I. Tamáska, O. Deparis et al., Mater. Sci. Eng. B 149, 259 (2008)

30. A.E. Seago, P. Brady, J.-P. Vigneron, T.D. Schultz, J.R. Soc, Interface 6, S165 (2009)

31. P. Vukusic, Science 325, 398 (2009)

32. V. Sharma, M. Crne, J.O. Park, M. Srinivasarao, Science 325, 449 (2009)

33. H.M. Whitney, M. Kolle, P. Andrew, L. Chittka, U. Steiner, B.J. Glover, Science 323, 130 (2009)

34. C.J. van der Kooi, A.G. Dyer, D.G. Stavenga, New Phytol. 205, 18 (2015)

35. P. Vukusic, J. Sambles, C. Lawrence, R. Wootton, Proc. R. Soc. Lond. Ser. B Biol. Sci. 266, 1403 (1999)

36. M. Giraldo, S. Yoshioka, C. Liu, D. Stavenga, J. Exp. Biol. 219, 3936 (2016)

37. E. De Tommasi, J. Gielis, A. Rogato, Mar. Genom. 35, 1 (2017)

38. S. Manago, G. Zito, A. Rogato, M. Casalino, E. Esposito, A.C. De Luca, E. De Tommasi, A.C.S. Appl, Mater. Interfaces 10, 12406 (2018)

39. S.B. Juhl, E.P. Chan, Y.-H. Ha, M. Maldovan, J. Brunton, V. Ward, T. Dokland, J. Kalmakoff, B. Farmer, E.L. Thomas et al., Adv. Funct. Mater. 16, 1086 (2006)

40. E. Rybicki, S. Afr, J. Sci. 86, 182 (1990)

41. P. Lupetti, G. Montesanto, S. Ciolfi, L. Marri, M. Gentile, E. Paccagnini, B.M. Lombardo, Tissue Cell 45, 321 (2013) 
42. P. Daszak, L. Berger, A.A. Cunningham, A.D. Hyatt, D.E. Green, R. Speare, Emerg. Infect. Dis. 5, 735 (1999)

43. C. Radloff, R.A. Vaia, J. Brunton, G.T. Bouwer, V.K. Ward, Nano Lett. 5, 1187 (2005)

44. J. Sun, B. Bhushan, J. Tong, RSC Adv. 3, 14862 (2013)

45. S. Kinoshita, S. Yoshioka, J. Miyazaki, Rep. Prog. Phys. 71, 076401 (2008)

46. F. Schenk, B.D. Wilts, D.G. Stavenga, Bioinspiration Biomim. 8, 045002 (2013)

47. J.W. Galusha, L.R. Richey, J.S. Gardner, J.N. Cha, M.H. Bartl, Phys. Rev. E 77, 050904 (2008)

48. R.T. Lee, G.S. Smith, Appl. Opt. 48, 4177 (2009)

49. V.E. Johansen, O.D. Onelli, L.M. Steiner, S. Vignolini, Photonics in nature: from order to disorder, in Functional surfaces in biology III (Springer, New York, 2017), pp. 53-89

50. S. Kinoshita, S. Yoshioka, Y. Fujii, N. Okamoto, Forma Tokyo 17, 103 (2002)

51. R.A. Potyrailo, H. Ghiradella, A. Vertiatchikh, K. Dovidenko, J.R. Cournoyer, E. Olson, Nat. Photonics 1, 123 (2007)

52. J. Gu, D. Zhang, Y. Tan, in Metallic Butterfly Wing Scales: Superstructures with High SurfaceEnhancement Properties for Optical Applications (Springer, New York, 2014)

53. N.L. Garrett, P. Vukusic, F. Ogrin, E. Sirotkin, C.P. Winlove, J. Moger, J. Biophotonics 2, 157 (2009)

54. D.H. Goldstein, Appl. Opt. 45, 7944 (2006)

55. A. Pace, Science 176, 678 (1972)

56. R. Meister, M.-A. Hallé, H. Dumoulin, P. Pieranski, Phys. Rev. E 54, 3771 (1996)

57. B.D. Wilts, H.M. Whitney, B.J. Glover, U. Steiner, S. Vignolini, Mater. Today Proc. 1, 177 (2014)

58. P. Vukusic, J. Sambles, C. Lawrence, Proc. R. Soc. Lond. Ser. B Biol. Sci. 271, S237 (2004)

59. W. Wang, W. Zhang, X. Fang, Y. Huang, Q. Liu, M. Bai, D. Zhang, Opt. Lett. 39, 4208 (2014)

60. W. Wang, W. Zhang, D. Zhang, G.P. Wang, RSC Adv. 7, 22749 (2017)

61. P. Vukusic, R. Sambles, C. Lawrence, G. Wakely, Appl. Opt. 40, 1116 (2001)

62. R.H. Siddique, G. Gomard, H. Hölscher, Nat. Commun. 6, 1 (2015)

63. T. Fuhrmann, S. Landwehr, M. El Rharbi-Kucki, M. Sumper, Appl. Phys. B 78, 257 (2004)

64. F.E. Round, R.M. Crawford, D.G. Mann, in Diatoms: Biology and Morphology of the Genera (Cambridge University Press, Cambridge, 2007)

65. K. Leblanc, B. Queguiner, F. Diaz, V. Cornet, M. Michel-Rodriguez, X.D. de Madron, C. Bowler, S. Malviya, M. Thyssen, G. Grégori et al., Nat. Commun. 9, 1 (2018)

66. J. Noyes, M. Sumper, P. Vukusic, J. Mater. Res. 23, 3229 (2008)

67. J.W. Goessling, Y. Su, C. Maibohm, M. Ellegaard, M. Kühl, J.R. Soc, Interface Focus 9, 20180031 (2019)

68. C.E. Hamm, R. Merkel, O. Springer, P. Jurkojc, C. Maier, K. Prechtel, V. Smetacek, Nature 421, 841 (2003)

69. M.S. Hale, J.G. Mitchell, Aquat. Microb. Ecol. 24, 287 (2001)

70. M.S. Hale, J.G. Mitchell, Nano Lett. 2, 657 (2002)

71. A. Waite, A. Fisher, P.A. Thompson, P.J. Harrison, Mar. Ecol. Prog. Ser. 157, 97 (1997)

72. E. De Tommasi, J. Spectrosc. 2016 (2016)

73. R.B. Hoover, M.J. Hoover, J. Ark. Acad. Sci. 24, 8 (1970)

74. D. Losic, J.G. Mitchell, R. Lal, N.H. Voelcker, Adv. Funct. Mater. 17, 2439 (2007)

75. M. Villani, V. Onesto, M. Coluccio, I. Valpapuram, R. Majewska, A. Alabastri, E. Battista, A. Schirato, D. Calestani, N. Coppedé et al., Micro Nano Eng. 2, 29 (2019)

76. J. Livage, Comptes Rendus Chim. 21, 969 (2018)

77. F. Sterrenburg, M.A. Tiffany, M.E.M. Del Castillo, J. Nanosci. Nanotechnol. 5, 140 (2005)

78. J.W. Goessling, W.P. Wardley, M. Lopez-Garcia, Adv. Sci. 7, 1903726 (2020)

79. M. Kucki, T. Fuhrmann-Lieker, J.R. Soc, Interface 9, 727 (2012)

80. T. Zhou, M. Tang, G. Xiang, B. Xiang, S. Hark, M. Martin, T. Baron, S. Pan, J.-S. Park, Z. Liu et al., Nat. Commun. 11, 1 (2020)

81. C. Jeffryes, T. Gutu, J. Jiao, G.L. Rorrer, Mater. Sci. Eng. C 28, 107 (2008)

82. C. Jeffryes, T. Gutu, J. Jiao, G.L. Rorrer, ACS Nano 2, 2103 (2008)

83. L. De Stefano, P. Maddalena, L. Moretti, I. Rea, I. Rendina, E. De Tommasi, V. Mocella, M. De Stefano, Superlattices Microstruct. 46, 84 (2009)

84. S. Yamanaka, R. Yano, H. Usami, N. Hayashida, M. Ohguchi, H. Takeda, K. Yoshino, J. Appl. Phys. 103, 074701 (2008)

85. K. Kieu, C. Li, Y. Fang, G. Cohoon, O. Herrera, M. Hildebrand, K. Sandhage, R.A. Norwood, Opt. Express 22, 15992 (2014) 
86. Y. D’Mello, D. Petrescu, J. Skoric, M. Campbell, M. Andrews, D.V. Plant, Characterization of the photonic response in Nitzschia filiformis phytoplankton, in Proc. of CLEO: QELS_Fundamental Science (Optical Society of America, Washington, 2018), pp. JTh2A-96

87. Y. D’Mello, S. Bernal, J. Skoric, D. Petrescu, M. Andrews, D.V. Plant, Photonic crystal behavior of Nitzschia filiformis phytoplankton for chlorophyll a photosynthesis, in Proc. of CLEO: QELS_Fundamental Science. (Optical Society of America, Washington, 2019), pp. JW2A-121

88. L. De Stefano, I. Rea, I. Rendina, M. De Stefano, L. Moretti, Opt. Express 15, 18082 (2007)

89. E. De Tommasi, I. Rea, V. Mocella, L. Moretti, M. De Stefano, I. Rendina, L. De Stefano, Opt. Express 18, 12203 (2010)

90. M.A. Ferrara, P. Dardano, L. De Stefano, I. Rea, G. Coppola, I. Rendina, R. Congestri, A. Antonucci, M. De Stefano, E. De Tommasi, PLoS One 9, e103750 (2014)

91. J. Romann, J.-C. Valmalette, A. Røyset, M.-A. Einarsrud, Opt. Lett. 40, 740 (2015)

92. C. Maibohm, S.M.M. Friis, M. Ellegaard, K. Rottwitt, Opt. Express 23, 9543 (2015)

93. E. De Tommasi, R. Congestri, P. Dardano, A.C. De Luca, S. Managò, I. Rea, M. De Stefano, Sci. Rep. 8, 1 (2018)

94. G. Di Caprio, G. Coppola, L.D. Stefano, M.D. Stefano, A. Antonucci, R. Congestri, E. De Tommasi, J. Biophotonics 7, 341 (2014)

95. D. Losic, J.G. Mitchell, N.H. Voelcker, Adv. Mater. 21, 2947 (2009)

96. R. Ragni, S. Cicco, D. Vona, G. Leone, G.M. Farinola, J. Mater. Res. 32, 279 (2017)

97. R. Ragni, S.R. Cicco, D. Vona, G.M. Farinola, Adv. Mater. 30, 1704289 (2018)

98. E.K. Payne, N.L. Rosi, C. Xue, C.A. Mirkin, Angewandte Chem. 117, 5192 (2005)

99. F. Ren, J. Campbell, G.L. Rorrer, A.X. Wang, IEEE J. Sel. Top. Quantum Electron. 20, 127 (2014)

100. J. Yang, L. Zhen, F. Ren, J. Campbell, G.L. Rorrer, A.X. Wang, J. Biophotonics 8, 659 (2015)

101. J.A. Kraai, A.X. Wang, G.L. Rorrer, Adv. Mater. Interfaces 2000191 (2020)

102. Y. Fang, V.W. Chen, Y. Cai, J.D. Berrigan, S.R. Marder, J.W. Perry, K.H. Sandhage, Adv. Funct. Mater. 22, 2550 (2012)

103. A. De Leebeeck, L.S. Kumar, V. De Lange, D. Sinton, R. Gordon, A.G. Brolo, Anal. Chem. 79, 4094 (2007)

104. J. Ji, J.G. O’Connell, D.J. Carter, D.N. Larson, Anal. Chem. 80, 2491 (2008)

105. E. De Tommasi, A. De Luca, L. Lavanga, P. Dardano, M. De Stefano, L. De Stefano, C. Langella, I. Rendina, K. Dholakia, M. Mazilu, Opt. Express 22, 27214 (2014)

106. M. Mazilu, J. Baumgartl, S. Kosmeier, K. Dholakia, Opt. Express 19, 933 (2011)

107. J. Baumgartl, S. Kosmeier, M. Mazilu, E.T. Rogers, N.I. Zheludev, K. Dholakia, Appl. Phys. Lett. 98, 181109 (2011)

108. X. Tsampoula, M. Mazilu, T. Vettenburg, F. Gunn-Moore, K. Dholakia, Photonics Res. 1, 42 (2013)

109. S. Kosmeier, M. Mazilu, J. Baumgartl, K. Dholakia, J. Opt. 13, 105707 (2011)

110. E. Van Putten, D. Akbulut, J. Bertolotti, W.L. Vos, A. Lagendijk, A. Mosk, Phys. Rev. Lett. 106, 193905 (2011)

111. E.T. Rogers, J. Lindberg, T. Roy, S. Savo, J.E. Chad, M.R. Dennis, N.I. Zheludev, Nat. Mater. 11, 432 (2012)

112. E.T. Rogers, S. Savo, J. Lindberg, T. Roy, M.R. Dennis, N.I. Zheludev, Appl. Phys. Lett. 102, 031108 (2013)

113. B. O'regan, M. Grätzel, Nature 353, 737 (1991)

114. M. Grätzel, Photoelectrochemical cells, in Materials For Sustainable Energy: A Collection of PeerReviewed Research and Review Articles from Nature Publishing Group (World Scientific, Singapore, 2011), pp. 26-32

115. C. Jeffryes, J. Campbell, H. Li, J. Jiao, G. Rorrer, Energy Environ. Sci. 4, 3930 (2011)

116. J. Toster, K.S. Iyer, W. Xiang, F. Rosei, L. Spiccia, C.L. Raston, Nanoscale 5, 873 (2013)

117. T. Bandara, M. Furlani, I. Albinsson, A. Wulff, B.-E. Mellander, Nanoscale Adv. 2, 199 (2020)

118. P. Bermel, C. Luo, L. Zeng, L.C. Kimerling, J.D. Joannopoulos, Opt. Express 15, 16986 (2007)

119. S.B. Mallick, M. Agrawal, P. Peumans, Opt. Express 18, 5691 (2010)

120. R. Dewan, M. Marinkovic, R. Noriega, S. Phadke, A. Salleo, D. Knipp, Opt. Express 17, 23058 (2009)

121. N.-N. Feng, J. Michel, L. Zeng, J. Liu, C.-Y. Hong, L.C. Kimerling, X. Duan, IEEE Trans Electron Dev. 54, 1926 (2007)

122. H.A. Atwater, A. Polman, Plasmonics for improved photovoltaic devices, in Materials for Sustainable Energy: A Collection of Peer-Reviewed Research and Review Articles from Nature Publishing Group (World Scientific, Singapore, 2011), pp. 1-11 
123. K. Aydin, V.E. Ferry, R.M. Briggs, H.A. Atwater, Nat. Commun. 2, 1 (2011)

124. C. Wang, S. Yu, W. Chen, C. Sun, Sci. Rep. 3, 1 (2013)

125. T. Qin, T. Gutu, J. Jiao, C.-H. Chang, G.L. Rorrer, J. Nanosci. Nanotechnol. 8, 2392 (2008)

126. M. Sprynskyy, P. Pomastowski, M. Hornowska, A. Król, K. Rafińska, B. Buszewski, Mater. Design 132, 22 (2017)

127. C. Itoh, K. Tanimura, N. Itoh, J. Phys. C Solid State Phys. 21, 4693 (1988)

128. M. Kucki, Biological photonic crystals: Diatoms: Dye functionalization of biological silica nanostructures, Ph.D. thesis (2009)

129. L. De Stefano, I. Rendina, M. De Stefano, A. Bismuto, P. Maddalena, Applied Physics Letters 87, 233902 (2005)

130. A. Setaro, S. Lettieri, P. Maddalena, L. De Stefano, Appl. Phys. Lett. 91, 051921 (2007)

131. S. Lettieri, A. Setaro, L. De Stefano, M. De Stefano, P. Maddalena, Adv. Funct. Mater. 18, 1257 (2008)

132. D.K. Gale, T. Gutu, J. Jiao, C.-H. Chang, G.L. Rorrer, Adv. Funct. Mater. 19, 926 (2009)

133. H.A. Bethe, Phys. Rev. 66, 163 (1944)

134. T.W. Ebbesen, H.J. Lezec, H. Ghaemi, T. Thio, P.A. Wolff, Nature 391, 667 (1998)

135. J.B. Pendry, Phys. Rev. Lett. 85, 3966 (2000)

136. N. Yu, F. Capasso, Nat. Mater. 13, 139 (2014)

137. A.V. Kildishev, A. Boltasseva, V.M. Shalaev, Science 339 (2013)

138. N. Meinzer, W.L. Barnes, I.R. Hooper, Nat. Photonics 8, 889 (2014)

139. A.E. Minovich, A.E. Miroshnichenko, A.Y. Bykov, T.V. Murzina, D.N. Neshev, Y.S. Kivshar, Laser Photonics Rev. 9, 195 (2015)

140. M. Hong, Sci. Bull. 61, 112 (2016)

141. L. Wu, Z. Yang, Y. Cheng, R. Gong, M. Zhao, Y. Zheng, J. Duan, X. Yuan, Appl. Phys. A 116, 643 (2014)

142. Q. Lévesque, M. Makhsiyan, P. Bouchon, F. Pardo, J. Jaeck, N. Bardou, C. Dupuis, R. Haïdar, J.-L. Pelouard, Appl. Phys. Lett. 104, 111105 (2014)

143. N. Yu, P. Genevet, M.A. Kats, F. Aieta, J.-P. Tetienne, F. Capasso, Z. Gaburro, Science 334, 333 (2011)

144. J. Shi, X. Fang, E.T. Rogers, E. Plum, K.F. MacDonald, N.I. Zheludev, Opt. Express 22, 21051 (2014)

145. V.S. Asadchy, Y. Ra’Di, J. Vehmas, S. Tretyakov, Phys. Rev. Lett. 114, 095503 (2015)

146. C.L. Holloway, D. Love, E. Kuester, A. Salandrino, N. Engheta, I.E.T. Microw, Antennas Propag. 2, 120 (2008)

147. C. Valagiannopoulos, A. Tukiainen, T. Aho, T. Niemi, M. Guina, S. Tretyakov, C. Simovski, Phys. Rev. B 91, 115305 (2015)

148. Y. Ra'di, V.S. Asadchy, S.A. Tretyakov, IEEE Trans. Antennas Propag. 61, 4606 (2013)

149. J.F. Nye, M.V. Berry, Proc. R. Soc. Lond. A Math. Phys. Sci. 336, 165 (1974)

150. E. Karimi, S.A. Schulz, I. De Leon, H. Qassim, J. Upham, R.W. Boyd, Light Sci. Appl. 3, e167 (2014)

151. J. Sun, X. Wang, T. Xu, Z.A. Kudyshev, A.N. Cartwright, N.M. Litchinitser, Nano Lett. 14, 2726 (2014)

152. J. Lin, P. Genevet, M.A. Kats, N. Antoniou, F. Capasso, Nano Lett. 13, 4269 (2013)

153. J. Hunt, J. Gollub, T. Driscoll, G. Lipworth, A. Mrozack, M.S. Reynolds, D.J. Brady, D.R. Smith, JOSA A 31, 2109 (2014)

154. G. Zheng, H. Mühlenbernd, M. Kenney, G. Li, T. Zentgraf, S. Zhang, Nature Nanotechnol. 10, 308 (2015)

155. C. Pfeiffer, A. Grbic, Physical Review B 91, 115408 (2015)

156. A. Li, S. Singh, D. Sievenpiper, Nanophotonics 7, 989 (2018)

157. S.M. Kamali, E. Arbabi, A. Arbabi, A. Faraon, Nanophotonics 7, 1041 (2018)

158. M. Pu, X. Ma, X. Li, Y. Guo, X. Luo, J. Mater. Chem. C 5, 4361 (2017)

159. F. Ding, Y. Yang, R.A. Deshpande, S.I. Bozhevolnyi, Nanophotonics 7, 1129 (2018)

160. M. Chen, M. Kim, A.M. Wong, G.V. Eleftheriades, Nanophotonics 7, 1207 (2018)

161. X. Ren, P.K. Jha, Y. Wang, X. Zhang, Nanophotonics 7, 1233 (2018)

162. B. Liang, J.-C. Cheng, C.-W. Qiu, Nanophotonics 7, 1191 (2018)

163. K. Achouri, M.A. Salem, C. Caloz, IEEE Trans. Antennas Propag. 63, 2977 (2015)

164. C. Pfeiffer, N.K. Emani, A.M. Shaltout, A. Boltasseva, V.M. Shalaev, A. Grbic, Nano Lett. 14, 2491 (2014)

165. C. Pfeiffer, A. Grbic, Phys. Rev. Appl. 2, 044011 (2014) 
166. P. Halevi, A. Boardman, Electromagnetic surface modes (1982)

167. A. Wokaun, Surface plasmons on smooth and rough surfaces and on gratings (1984)

168. M.C. Hutley, (1982)

169. E. Kretschmann, H. Raether, Z. Naturforsch, A 23, 2135 (1968)

170. A. Otto, Zeitschrift für Phys. A Hadrons Nuclei 216, 398 (1968)

171. J. Homola, M. Piliarik, Surface plasmon resonance (spr) sensors, in Surface plasmon resonance based sensors (Springer, New York, 2006), pp. 45-67

172. C. Bohren, D (Huffman, New York, 1983)

173. U. Kreibig, M. Vollmer, Optical Properties of Metal Clusters, vol. 25 (Springer, New York, 2013)

174. T.R. Jensen, M.D. Malinsky, C.L. Haynes, R.P. Van Duyne, J. Phys. Chem. B 104, 10549 (2000)

175. X. Zhang, E.M. Hicks, J. Zhao, G.C. Schatz, R.P. Van Duyne, Nano Lett. 5, 1503 (2005)

176. G. Si, Y. Zhao, J. Lv, M. Lu, F. Wang, H. Liu, N. Xiang, T.J. Huang, A.J. Danner, J. Teng et al., Nanoscale 5, 6243 (2013)

177. F. Hao, C.L. Nehl, J.H. Hafner, P. Nordlander, Nano Lett. 7, 729 (2007)

178. P.S. Kumar, I. Pastoriza-Santos, B. Rodríguez-González, F.J.G. De Abajo, L.M. Liz-Marzán, Nanotechnology 19, 015606 (2007)

179. F.J. Rodríguez-Fortuño, M. Martínez-Marco, B. Tomás-Navarro, R. Ortuño, J. Martí, A. Martínez, P.J. Rodríguez-Cantó, Appl. Phys. Lett. 98, 133118 (2011)

180. V. Di Meo, A. Caporale, A. Crescitelli, M. Janneh, E. Palange, A. De Marcellis, M. Portaccio, M. Lepore, I. Rendina, M. Ruvo et al., Sens. Actuators B Chem. 286, 600 (2019)

181. F. Wang, Y.R. Shen, Phys. Rev. Lett. 97, 206806 (2006)

182. E. Heilweil, R. Hochstrasser, J. Chem. Phys. 82, 4762 (1985)

183. J.R. Krenn, A. Dereux, J.-C. Weeber, E. Bourillot, Y. Lacroute, J.-P. Goudonnet, G. Schider, W. Gotschy, A. Leitner, F.R. Aussenegg et al., Phys. Rev. Lett. 82, 2590 (1999)

184. E. Prodan, C. Radloff, N.J. Halas, P. Nordlander, Science 302, 419 (2003)

185. N.J. Halas, S. Lal, W.-S. Chang, S. Link, P. Nordlander, Chem. Rev. 111, 3913 (2011)

186. L. Panina, A. Grigorenko, D. Makhnovskiy, Phys. Rev. B 66, 155411 (2002)

187. A.L. Koh, K. Bao, I. Khan, W.E. Smith, G. Kothleitner, P. Nordlander, S.A. Maier, D.W. McComb, ACS Nano 3, 3015 (2009)

188. I. Zoric, M. Zach, B. Kasemo, C. Langhammer, ACS Nano 5, 2535 (2011)

189. S. Rodriguez, M. Schaafsma, A. Berrier, J.G. Rivas, Phys. B Condens. Matter 407, 4081 (2012)

190. N. Félidj, J. Aubard, G. Lévi, J.R. Krenn, G. Schider, A. Leitner, F. Aussenegg, Phys. Rev. B 66, 245407 (2002)

191. N. Lawandy, Appl. Phys. Lett. 85, 5040 (2004)

192. N.K. Emani, A.V. Kildishev, V.M. Shalaev, A. Boltasseva, Nanophotonics 1, 214 (2015)

193. S. Kawata, Y. Inouye, P. Verma, Nat. Photonics 3, 388 (2009)

194. Z. Li, E. Palacios, S. Butun, K. Aydin, Nano Lett. 15, 1615 (2015)

195. S. Choudhury, U. Guler, A. Shaltout, V.M. Shalaev, A.V. Kildishev, A. Boltasseva, Adv. Opt. Mater. 5, 1700196 (2017)

196. Y. Zhao, A. Alù, Phys. Rev. B 84, 205428 (2011)

197. N. Yu, F. Aieta, P. Genevet, M.A. Kats, Z. Gaburro, F. Capasso, Nano Lett. 12, 6328 (2012)

198. F. Ding, Z. Wang, S. He, V.M. Shalaev, A.V. Kildishev, ACS Nano 9, 4111 (2015)

199. F. Yue, D. Wen, J. Xin, B.D. Gerardot, J. Li, X. Chen, ACS Photonics 3, 1558 (2016)

200. X. Ni, Z.J. Wong, M. Mrejen, Y. Wang, X. Zhang, Science 349, 1310 (2015)

201. S. Ishii, A.V. Kildishev, V.M. Shalaev, K.-P. Chen, V.P. Drachev, Opt. Lett. 36, 451 (2011)

202. J.N. Anker, W.P. Hall, O. Lyandres, N.C. Shah, J. Zhao, R.P. Van Duyne, Biosensing with plasmonic nanosensors, in Nanoscience and Technology: A Collection of Reviews from Nature Journals (World Scientific, Singapore, 2010), pp. 308-319

203. A. Kabashin, P. Evans, S. Pastkovsky, W. Hendren, G. Wurtz, R. Atkinson, R. Pollard, V. Podolskiy, A. Zayats, Nat. Mater. 8, 867 (2009)

204. V. Kravets, F. Schedin, R. Jalil, L. Britnell, R. Gorbachev, D. Ansell, B. Thackray, K. Novoselov, A. Geim, A.V. Kabashin et al., Nat. Mater. 12, 304 (2013)

205. K.V. Sreekanth, Y. Alapan, M. ElKabbash, E. Ilker, M. Hinczewski, U.A. Gurkan, A. De Luca, G. Strangi, Nat. Mater. 15, 621 (2016)

206. J.B. Khurgin, Nat. Nanotechnol. 10, 2 (2015)

207. X. Han, K. Liu, C. Sun, Materials 12, 1411 (2019) 
208. P. Larkin, Infrared and Raman Spectroscopy: Principles and Spectral Interpretation (Elsevier, Amsterdam, 2017)

209. S. Nie, S.R. Emory, Science 275, 1102 (1997)

210. K. Kneipp, Y. Wang, H. Kneipp, L.T. Perelman, I. Itzkan, R.R. Dasari, M.S. Feld, Phys. Rev. Lett. 78, 1667 (1997)

211. S. Schlücker, Angew. Chem. Int. Ed. 53, 4756 (2014)

212. J. Vogt, C. Huck, F. Neubrech, A. Toma, D. Gerbert, A. Pucci, Phys. Chem. Chem. Phys. 17, 21169 (2015)

213. R. Adato, S. Aksu, H. Altug, Mater. Today 18, 436 (2015)

214. F. Neubrech, C. Huck, K. Weber, A. Pucci, H. Giessen, Chem. Rev. 117, 5110 (2017)

215. T.H. Reilly, S.-H. Chang, J.D. Corbman, G.C. Schatz, K.L. Rowlen, J. Phys. Chem. C 111, 1689 (2007)

216. S. Pang, T. Yang, L. He, TrAC. Trends Anal. Chem. 85, 73 (2016)

217. D.L. Jeanmaire, R.P. Van Duyne, J. Electroanal. Chem. Interfacial Electrochem. 84, 1 (1977)

218. M.G. Albrecht, J.A. Creighton, J. Am. Chem. Soc. 99, 5215 (1977)

219. A. Hartstein, J. Kirtley, J. Tsang, Phys. Rev. Lett. 45, 201 (1980)

220. J.P. Camden, J.A. Dieringer, J. Zhao, R.P. Van Duyne, Acc. Chem. Res. 41, 1653 (2008)

221. Y. Nishikawa, T. Nagasawa, K. Fujiwara, M. Osawa, Vib. Spectrosc. 6, 43 (1993)

222. M. Fan, G.F. Andrade, A.G. Brolo, Anal. Chim. Acta 693, 7 (2011)

223. A.B. Serrano-Montes, J. Langer, M. Henriksen-Lacey, D. Jimenez de Aberasturi, D.M. Solís, J.M. Taboada, F. Obelleiro, K. Sentosun, S. Bals, A. Bekdemir et al., J. Phys. Chem. C 120, 20860 (2016)

224. T.B. Neves, S.M. Landi, L.A. Sena, B.S. Archanjo, G.F. Andrade, RSC Adv. 5, 59373 (2015)

225. K.A. Willets, R.P. Van Duyne, Annu. Rev. Phys. Chem. 58, 267 (2007)

226. P. Zhang, S. Yang, L. Wang, J. Zhao, Z. Zhu, B. Liu, J. Zhong, X. Sun, Nanotechnology 25, 245301 (2014)

227. G. Barbillon, A. Ivanov, A.K. Sarychev, Nanomaterials 9, 1588 (2019)

228. E.B. Santos, C. Valsecchi, J.L. Gonçalves, L.F. Ávila, J.W. Menezes, Sensors 19, 4394 (2019)

229. S.-H. Chang, S.K. Gray, G.C. Schatz, Opt. Express 13, 3150 (2005)

230. C. Lu, Z. Qi, X. Hu, Z. Ni, Y. Cui, Opt. Commun. 452, 494 (2019)

231. N. Kazemi-Zanjani, M. Shayegannia, R. Prinja, A.O. Montazeri, A. Mohammadzadeh, K. Dixon, S. Zhu, P.R. Selvaganapathy, A. Zavodni, N. Matsuura et al., Adv. Opt. Mater. 6, 1701136 (2018)

232. O. Guselnikova, P. Postnikov, A. Pershina, V. Svorcik, O. Lyutakov, Appl. Surf. Sci. 470, 219 (2019)

233. W. Yue, Z. Wang, J. Whittaker, F. Lopez-royo, Y. Yang, A.V. Zayats, J. Mater. Chem. C 5, 4075 (2017)

234. J. Zhang, M. Irannejad, B. Cui, Plasmonics 10, 831 (2015)

235. L. Feng, R. Ma, Y. Wang, D. Xu, D. Xiao, L. Liu, N. Lu, Nano Res. 8, 3715 (2015)

236. P. Liao, A. Wokaun, J. Chem. Phys. 76, 751 (1982)

237. L. Petti, R. Capasso, M. Rippa, M. Pannico, P. La Manna, G. Peluso, A. Calarco, E. Bobeico, P. Musto, Vib. Spectrosc. 82, 22 (2016)

238. J.-F. Bryche, A. Tsigara, B. Bélier, M.L. de la Chapelle, M. Canva, B. Bartenlian, G. Barbillon, Sens. Actuators B Chem. 228, 31 (2016)

239. L. Zhang, W. Zhang, F. Lu, Z. Yang, T. Xue, M. Liu, C. Meng, P. Li, D. Mao, T. Mei et al., Photonics Res. 7, 1447 (2019)

240. W. Yue, T. Gong, X. Long, V. Kravets, P. Gao, M. Pu, C. Wang, Sens. Actuators B Chem. 322, 128563 (2020)

241. N. Chamuah, G.P. Vaidya, A.M. Joseph, P. Nath, Plasmonics 12, 1353 (2017)

242. N.A. Cinel, S. Cakmakyapan, G. Ertaş, E. Özbay, I.E.E.E.J. Sel, Topics. Quantum Electron. 19, 4601605 (2012)

243. G.F. Andrade, Q. Min, R. Gordon, A.G. Brolo, J. Phys. Chem. C 116, 2672 (2012)

244. M.S. Davis, W. Zhu, T. Xu, J.K. Lee, H.J. Lezec, A. Agrawal, Nat. Commun. 8, 1 (2017)

245. A. Gopinath, S.V. Boriskina, W.R. Premasiri, L. Ziegler, B.M. Reinhard, L. Dal Negro, Nano Lett. 9 , $3922(2009)$

246. D. Radziuk, H. Moehwald, Phys. Chem. Chem. Phys. 17, 21072 (2015)

247. M. Rippa, R. Castagna, M. Pannico, P. Musto, E. Bobeico, J. Zhou, L. Petti, Opt. Data Process. Storage 3, 54 (2017)

248. R. Adato, A.A. Yanik, J.J. Amsden, D.L. Kaplan, F.G. Omenetto, M.K. Hong, S. Erramilli, H. Altug, Proc. Natl. Acad. Sci. 106, 19227 (2009) 
249. C. Huck, A. Toma, F. Neubrech, M. Chirumamilla, J. Vogt, F. De Angelis, A. Pucci, ACS Photonics 2, 497 (2015)

250. M. Hentschel, T. Weiss, S. Bagheri, H. Giessen, Nano Lett. 13, 4428 (2013)

251. C. Huck, J. Vogt, M. Sendner, D. Hengstler, F. Neubrech, A. Pucci, ACS Photonics 2, 1489 (2015)

252. R. Semenyshyn, M. Hentschel, C. Huck, J. Vogt, F. Weiher, H. Giessen, F. Neubrech, ACS Sens. 4, 1966 (2019)

253. E. Aslan, M. Turkmen, Sens. Actuators A Phys. 259, 127 (2017)

254. I. Mbomson, S. Tabor, B. Lahiri, G. Sharp, S. McMeekin, R. De La Rue, N. Johnson, Biomed. Opt. Express 8, 395 (2017)

255. S. Bagheri, K. Weber, T. Gissibl, T. Weiss, F. Neubrech, H. Giessen, ACS Photonics 2, 779 (2015)

256. L. Dong, X. Yang, C. Zhang, B. Cerjan, L. Zhou, M.L. Tseng, Y. Zhang, A. Alabastri, P. Nordlander, N.J. Halas, Nano Lett. 17, 5768 (2017)

257. L.V. Brown, X. Yang, K. Zhao, B.Y. Zheng, P. Nordlander, N.J. Halas, Nano Lett. 15, 1272 (2015)

258. V. Di Meo, A. Crescitelli, M. Moccia, A. Sandomenico, A.M. Cusano, M. Portaccio, M. Lepore, V. Galdi, E. Esposito, Nanophotonics 9, 3921 (2020)

259. D. Rodrigo, A. Tittl, A. John-Herpin, O. Limaj, H. Altug, ACS Photonics 5, 4903 (2018)

260. J.W. Petefish, A.C. Hillier, Anal. Chem. 87, 10862 (2015)

261. E. Aslan, E. Aslan, R. Wang, M.K. Hong, S. Erramilli, M. Turkmen, O.G. Saracoglu, L. Dal Negro, ACS Photonics 3, 2102 (2016)

262. G.Q. Wallace, M. Tabatabaei, R. Hou, M.J. Coady, P.R. Norton, T.S. Simpson, S.M. Rosendahl, A. Merlen, F. Lagugné-Labarthet, ACS Photonics 3, 1723 (2016)

263. G. Kostovski, P.R. Stoddart, A. Mitchell, Adv. Mater. 26, 3798 (2014)

264. A. Cusano, M. Consales, M. Pisco, A. Crescitelli, A. Ricciardi, E. Esposito, A. Cutolo, Lab on fiber technology and related devices, part I: A new technological scenario; lab on fiber technology and related devices, part II: The impact of the nanotechnologies, in Proc. of International Conference on Applications of Optics and Photonics, vol. 8001 (International Society for Optics and Photonics, 2011)

265. M. Principe, M. Consales, A. Micco, A. Crescitelli, G. Castaldi, E. Esposito, V. La Ferrara, A. Cutolo, V. Galdi, A. Cusano, Light Sci. Appl. 6, e16226 (2017)

266. P. Malara, A. Crescitelli, V. Di Meo, A. Giorgini, S. Avino, E. Esposito, A. Ricciardi, A. Cusano, I. Rendina, P. De Natale et al., Sens. Actuators B Chem. 273, 1587 (2018)

267. A.N. Broers, Philos. Trans. R Soc. Lond. Ser. A Phys. Eng. Sci. 353, 291 (1995)

268. A. Broers, W. Molzen, J. Cuomo, N. Wittels, Appl. Phys. Lett. 29, 596 (1976)

269. A. Crescitelli, A. Ricciardi, M. Consales, E. Esposito, C. Granata, V. Galdi, A. Cutolo, A. Cusano, Adv. Funct. Mater. 22, 4389 (2012)

270. S. Matsui, Y. Kojima, Y. Ochiai, Appl. Phys. Lett. 53, 868 (1988)

271. K. Arshak, M. Mihov, J Optoelectron Adv. Mater. 7, 193 (2005)

272. K. Gamo, Microelectron. Eng. 32, 159 (1996)

273. S. Matsui, K. Mori, K. Saigo, T. Shiokawa, K. Toyoda, S. Namba, J. Vac. Sci. Technol. B Microelectron. Process. Phenom. 4, 845 (1986)

274. M. Horák, K. Bukvišová, V. Švarc, J. Jaskowiec, V. Křápek, T. Šikola, Sci. Rep. 8, 1 (2018)

275. M. Colburn, S.C. Johnson, M.D. Stewart, S. Damle, T.C. Bailey, B. Choi, M. Wedlake, T.B. Michaelson, S. Sreenivasan, J.G. Ekerdt et al., Step and flash imprint lithography: a new approach to high-resolution patterning, in proc. of Emerging Lithographic Technologies III, vol. 3676 (International Society for Optics and Photonics, 1999), pp. 379-389

276. H. Hiroshima, M. Komuro, N. Kasahara, Y. Kurashima, J. Taniguchi, Jpn. J. Appl. Phys. 42,3849 (2003)

277. A. Fuchs, M. Bender, U. Plachetka, U. Hermanns, H. Kurz, J. Vac. Sci. Technol. B Microelectron. Nanometer Struct. Process. Meas. Phenom. 23, 2925 (2005)

278. C. Farcau, D. Marconi, A. Colniţă, I. Brezeţtean, L. Barbu-Tudoran, Nanomaterials 9, 1519 (2019)

279. S. Kasani, K. Curtin, N. Wu, Nanophotonics 8, 2065 (2019)

280. J.H. Moon, S.-M. Yang, D.J. Pine, W.-S. Chang, Appl. Phys. Lett. 85, 4184 (2004)

281. C. Valsecchi, L.E. Gomez Armas, J. Weber de Menezes, Sensors 19, 2182 (2019)

282. I. Byun, J. Kim, J. Micromech. Microeng. 20, 055024 (2010)

283. M. Helgert, M. Burkhardt, K. Rudolf, R. Steiner, R. Brunner, High-frequent structures generated by interference lithography in the duv, in Proc. of Diffractive Optics and Micro-Optics (Optical Society of America, Washington, 2004) 
284. T.B. O'Reilly, H.I. Smith, J. Vac. Sci. Technol. B Microelectron. Nanometer Struct. Process. Meas. Phenom. 26, 2131 (2008)

285. M. Grzelczak, J. Vermant, E.M. Furst, L.M. Liz-Marzán, ACS Nano 4, 3591 (2010)

286. M. Karg, T.A. König, M. Retsch, C. Stelling, P.M. Reichstein, T. Honold, M. Thelakkat, A. Fery, Mater. Today 18, 185 (2015)

287. P.N. Bartlett, P.R. Birkin, M.A. Ghanem, Chem. Commun. 1671 (2000)

288. J.C. Hulteen, D.A. Treichel, M.T. Smith, M.L. Duval, T.R. Jensen, R.P. Van Duyne, J. Phys. Chem. B 103, 3854 (1999)

289. P. Pieranski, Phys. Rev. Lett. 45, 569 (1980)

290. R.C. Rossi, M.X. Tan, N.S. Lewis, Appl. Phys. Lett. 77, 2698 (2000)

291. J.C. Hulteen, R.P. Van Duyne, J. Vac Sci. Technol. A Vac. Surf. Films 13, 1553 (1995)

292. W.-d RUAN, Z.-c LÜ, J. Nan, C.-x WANG, Z. Bing, J.-h ZHANG, Chem. Res. Chin. Univ. 23, 712 (2007)

293. S. Kasani, P. Zheng, N. Wu, J. Phys. Chem. C 122, 13443 (2017)

294. R.D. Piner, J. Zhu, F. Xu, S. Hong, C.A. Mirkin, Science 283, 661 (1999)

295. H. Zhang, N.A. Amro, S. Disawal, R. Elghanian, R. Shile, J. Fragala, Small 3, 81 (2007)

296. D.S. Ginger, H. Zhang, C.A. Mirkin, Angew. Chem. Int. Ed. 43, 30 (2004)

297. C.A. Mirkin, ACS Nano 1, 79 (2007)

298. K. Salaita, Y. Wang, J. Fragala, R.A. Vega, C. Liu, C.A. Mirkin, Angew. Chem. 118, 7378 (2006)

299. R. Verre, M. Svedendahl, N.O. Lank, Z. Yang, G. Zengin, T. Antosiewicz, M. Kall, Nano Lett. 16, 98 (2016)

300. P. Gutruf, C. Zou, W. Withayachumnankul, M. Bhaskaran, S. Sriram, C. Fumeaux, ACS Nano 10, $133(2016)$

301. K.J. Vahala, Nature 424, 839 (2003)

302. C.F. Bohren, D.R. Huffman, Absorption and Scattering of Light by Small Particles (Wiley, New York, 2008)

303. A.A. Bogdanov, K.L. Koshelev, P.V. Kapitanova, M.V. Rybin, S.A. Gladyshev, Z.F. Sadrieva, K.B. Samusev, Yu.S. Kivshar, M.F. Limonov, 1 (2018). arXiv.org

304. F.J. García de Abajo, Rev. Mod. Phys. 79, 1267 (2007)

305. C. Blanchard, J.-P. Hugonin, C. Sauvan, Phys. Rev. B 94, 155303 (2016)

306. N. Bleistein, J.K. Cohen, J. Math. Phys. 18, 194 (1977)

307. M. Notomi, 62, 696 (2000)

308. V. Mocella, S. Cabrini, a Chang, P. Dardano, L. Moretti, I. Rendina, D. Olynick, B. Harteneck, S. Dhuey, Phys. Rev. Lett. 102, 133902 (2009)

309. S. Romano, S. Cabrini, I. Rendina, V. Mocella, Light Sci. Appl. 3, e120 (2014)

310. E. De Tommasi, A. Chiara De Luca, S. Cabrini, I. Rendina, S. Romano, V. Mocella, Appl. Phys. Lett. $102(2013)$

311. H. Raether, Surface Plasmons on Smooth and Rough Surfaces and on Gratings (Springer, New York, 1988)

312. J. von Neumann, E.P. Wigner, Phys. Zeitschrift 30, 465 (1929)

313. Z. Yu, X. Sun, Light Sci. Appl. 9, 1 (2020)

314. C. Linton, P. McIver, Wave Motion 45, 16 (2007)

315. R. Porter, D.V. Evans, Wave Motion 43, 29 (2005)

316. E.N. Bulgakov, A.F. Sadreev, Phys. Rev. B 78, 0751051 (2008)

317. Y. Plotnik, O. Peleg, F. Dreisow, M. Heinrich, S. Nolte, A. Szameit, M. Segev, Phys. Rev. Lett. 107, $183901(2011)$

318. M.I. Molina, A.E. Miroshnichenko, Y.S. Kivshar, Phys. Rev. Lett. 108, 0704011 (2012)

319. C.W. Hsu, B. Zhen, J. Lee, S.-L. Chua, S.G. Johnson, J.D. Joannopoulos, M. Soljačić, Nature 499, 188 (2013)

320. B. Zhen, C.W. Hsu, L. Lu, aD. Stone, M. Soljačić, Phys. Rev. Lett. 113, 2574011 (2014)

321. V. Mocella, S. Romano, Phys. Rev. B 92, 1 (2015)

322. C.W. Hsu, B. Zhen, A.D. Stone, J.D. Joannopoulos, M. Soljacic, Nat. Rev. Mater. 1 (2016)

323. E. Penzo, S. Romano, Y. Wang, S. Dhuey, L. Dal Negro, V. Mocella, S. Cabrini, J. Vac. Sci. Technol. B 35, 06G401 (2017)

324. E.N. Bulgakov, D.N. Maksimov, J. Opt. Soc. Am. B 35, 2443 (2018)

325. K. Koshelev, A. Bogdanov, Y. Kivshar, Sci. Bull. 64, 836 (2019) 
326. A. Tittl, A. Leitis, M. Liu, F. Yesilkoy, D.-Y. Choi, D.N. Neshev, Y.S. Kivshar, H. Altug, Science 360, 1105 (2018)

327. F. Yesilkoy, E.R. Arvelo, Y. Jahani, M. Liu, A. Tittl, V. Cevher, Y. Kivshar, H. Altug, Nat. Photonics 13, 390 (2019)

328. K. Koshelev, G. Favraud, A. Bogdanov, Y. Kivshar, A. Fratalocchi, Nanophotonics 8, 725 (2019)

329. G. Zito, S. Romano, S. Cabrini, G. Calafiore, A.C. De Luca, E. Penzo, V. Mocella, Optica 6, 1305 (2019)

330. S. Romano, M. Mangini, E. Penzo, S. Cabrini, A.C. De Luca, I. Rendina, V. Mocella, G. Zito, ACS Nano 14, 15417 (2020)

331. A. Kodigala, T. Lepetit, Q. Gu, B. Bahari, Y. Fainman, B. Kanté, Nature 541, 196 (2017)

332. S.T. Ha, Y.H. Fu, N.K. Emani, Z. Pan, R.M. Bakker, R. Paniagua-Domínguez, A.I. Kuznetsov, Nat. Nanotechnol. 13, 1042 (2018)

333. C. Huang, C. Zhang, S. Xiao, Y. Wang, Y. Fan, Y. Liu, N. Zhang, G. Qu, H. Ji, J. Han et al., Science 367, 1018 (2020)

334. M. Wu, S.T. Ha, S. Shendre, E.G. Durmusoglu, W.-K. Koh, D.R. Abujetas, J.A. Sánchez-Gil, R. Paniagua-Dominguez, H.V. Demir, A.I. Kuznetsov, Nano Lett. 20, 6005 (2020)

335. A.P. Anthur, H. Zhang, R. Paniagua-Dominguez, D.A. Kalashnikov, S.T. Ha, T.W. Maß, A.I. Kuznetsov, L. Krivitsky, Nano Lett. (2020)

336. N. Bernhardt, K. Koshelev, S.J. White, K.W.C. Meng, J.E. Froch, S. Kim, T.T. Tran, D.-Y. Choi, Y. Kivshar, A.S. Solntsev, Nano Lett. 20, 5309 (2020)

337. K. Koshelev, Y. Tang, K. Li, D.-Y. Choi, G. Li, Y. Kivshar, ACS Photonics 6, 1639 (2019)

338. Z. Liu, Y. Xu, Y. Lin, J. Xiang, T. Feng, Q. Cao, J. Li, S. Lan, J. Liu, Phys. Rev. Lett. 123, 253901 (2019)

339. S. Romano, G. Zito, S. Managò, G. Calafiore, E. Penzo, S. Cabrini, A.C. De Luca, V. Mocella, J. Phys. Chem. C 122, 19738 (2018)

340. C. Huang, C. Zhang, S. Xiao, Y. Wang, Y. Fan, Y. Liu, N. Zhang, G. Qu, H. Ji, J. Han, L. Ge, Y. Kivshar, Q. Song, Science 367, 1018 (2020)

341. N. Bernhardt, K. Koshelev, S.J. White, K.W.C. Meng, J.E. Froch, S. Kim, T.T. Tran, D.-Y. Choi, Y. Kivshar, A.S. Solntsev, Nano Lett. 20, 5309 (2020)

342. E.N. Bulgakov, D.N. Maksimov, Phys. Rev. A 96, 063833 (2017)

343. X. Yin, J. Jin, M. Soljačić, C. Peng, B. Zhen, Nature 580, 467 (2020)

344. G. Zito, S. Romano, S. Cabrini, G. Calafiore, A.C. De Luca, E. Penzo, V. Mocella, Optica 6, 1305 (2019)

345. B. Wang, W. Liu, M. Zhao, J. Wang, Y. Zhang, A. Chen, F. Guan, X. Liu, L. Shi, J. Zi, Nat. Photonics 14, 623 (2020)

346. Z. Yu, X. Xi, J. Ma, H.K. Tsang, C.-L. Zou, X. Sun, Optica 6, 1342 (2019)

347. T. Wang, Z. Li, X. Zhang, Photonics Res. 7, 341 (2019)

348. A.N. Poddubny, D.A. Smirnova, (2018). arXiv:1808.04811

349. S.I. Azzam, A.V. Kildishev, Adv. Opt. Mater. 2001469

350. D. Marinica, a Borisov, S. Shabanov, Phys. Rev. Lett. 100, 183902 (2008)

351. C. Wei Hsu, B. Zhen, S.-L. Chua, S.G. Johnson, J.D. Joannopoulos, M. Soljačić, Light Sci. Appl. 2, e84 (2013)

352. E.N. Bulgakov, A.F. Sadreev, Phys. Rev. A 90, 053801 (2014)

353. Z. Hu, Y.Y. Lu, J. Opt. 17, 065601 (2015)

354. L. Yuan, Y.Y. Lu, J. Phys. B At. Mol. Opt. Phys. 50, 05LT01 (2017)

355. K. Koshelev, A. Bogdanov, Y. Kivshar, Sci. Bull. 64, 836 (2019)

356. S.I. Azzam, V.M. Shalaev, A. Boltasseva, A.V. Kildishev, Phys. Rev. Lett. 121, 253901 (2018)

357. N. Gallo, M. Molina, J. Phys. A Math. Theor. 48, 045302 (2014)

358. H.M. Doeleman, F. Monticone, W. den Hollander, A. Alù, A.F. Koenderink, Nat. Photonics 12, 397 (2018)

359. C.W. Hsu, B. Zhen, M. Soljačić, A.D. Stone, (2017). arXiv:1708.02197

360. Z. Sadrieva, K. Frizyuk, M. Petrov, Y. Kivshar, A. Bogdanov, Phys. Rev. B 100, 115303 (2019)

361. M.F. Picardi, A.V. Zayats, F.J. Rodríguez-Fortuño, Phys. Rev. Lett. 120, 117402 (2018)

362. J. Jin, X. Yin, L. Ni, M. Soljačić, B. Zhen, C. Peng, Nature 574, 501 (2019)

363. A. Kodigala, T. Lepetit, Q. Gu, B. Bahari, Y. Fainman, B. Kanté, Nature 541, 196 (2017)

364. K. Koshelev, S. Kruk, E. Melik-Gaykazyan, J.-H. Choi, A. Bogdanov, H.-G. Park, Y. Kivshar, Science 367, 288 (2020) 
365. S. Romano, A. Lamberti, M. Masullo, E. Penzo, S. Cabrini, I. Rendina, V. Mocella, Materials 11, 1 (2018)

366. M.V. Rybin, K.L. Koshelev, Z.F. Sadrieva, K.B. Samusev, A.A. Bogdanov, M.F. Limonov, Y.S. Kivshar, Phys. Rev. Lett. 119, 243901 (2017)

367. V. Mylnikov, S.T. Ha, Z. Pan, V. Valuckas, R. Paniagua-Dominguez, H.V. Demir, A.I. Kuznetsov, ACS Nano 14, 7338 (2020)

368. E. Bulgakov, K. Pichugin, A. Sadreev, (2020). arXiv:2005.05554

369. Y. Liang, K. Koshelev, F. Zhang, H. Lin, S. Lin, J. Wu, B. Jia, Y. Kivshar, Nano Lett. 20, 6351 (2020)

370. V. Mocella, S. Romano, Phys. Rev. B 92, 155117 (2015)

371. K. Koshelev, S. Lepeshov, M. Liu, A. Bogdanov, Y. Kivshar, Phys. Rev. Lett. 121, 193903 (2018)

372. L. Marrucci, C. Manzo, D. Paparo, Phys. Rev. Lett. 96, 163905 (2006)

373. B. Zhen, S.-L. Chua, J. Lee, A.W. Rodriguez, X. Liang, S.G. Johnson, J.D. Joannopoulos, M. Soljacic, O. Shapira, Proc. Natl. Acad. Sci. USA 110, 13711 (2013)

374. S.A. Maier, Opt. Express 14, 1957 (2006)

375. Y. Ra'di, A. Krasnok, A. Alú, ACS Photonics (2020)

376. L. Carletti, K. Koshelev, C. De Angelis, Y. Kivshar, Phys. Rev. Lett. 121, 033903 (2018)

377. L. Carletti, S.S. Kruk, A.A. Bogdanov, C. De Angelis, Y. Kivshar, Phys. Rev. Res. 1, 023016 (2019)

378. G. Zograf, K. Koshelev, A. Zalogina, V. Korolev, D.-Y. Choi, M. Zurch, C. Spielmann, B. LutherDavies, D. Kartashov, S. Makarov et al., (2020). arXiv:2008.11481

379. S. Romano, G. Zito, S. Torino, S. Cabrini, I. Rendina, G. Coppola, G. Calafiore, E. Penzo, V. Mocella, Photonics Res. 6, 726 (2018)

380. Y. Liu, W. Zhou, Y. Sun, Sensors (Switzerland) 17 (2017)

381. S. Romano, G. Zito, S.N. Lara Yépez, S. Cabrini, E. Penzo, G. Coppola, I. Rendina, V. Mocella, Opt. Express 27, 18776 (2019)

382. D.N. Maksimov, V.S. Gerasimov, S. Romano, S.P. Polyutov, Opt. Express 28, 38907 (2020)

383. Y.-N. Zhang, Y. Zhao, R.-Q. Lv, Sens. Actuators A Phys. 233, 374 (2015)

384. A. Di Falco, L. O'faolain, T. Krauss, Appl. Phys. Lett. 94, 063503 (2009)

385. A. Leitis, A. Tittl, M. Liu, B.H. Lee, M.B. Gu, Y.S. Kivshar, H. Altug, Sci. Adv. 5, eaaw2871 (2019)

386. R.E. Raab, O.L. De Lange, O.L. de Lange et al., Multipole Theory in Electromagnetism: Classical, Quantum, and Symmetry Aspects, with Applications, vol. 128 (Oxford University Press on Demand, Oxford, 2005)

387. V.M. Dubovik, V.V. Tugushev, Phys. Rep. 187, 145 (1990)

388. K.V. Baryshnikova, D.A. Smirnova, B.S. Luk'yanchuk, Y.S. Kivshar, Adv. Opt. Mater. 7, 1801350 (2019)

389. N. Talebi, S. Guo, P.A. van Aken, Nanophotonics 7, 93 (2018)

390. Y. Yang, S.I. Bozhevolnyi, Nanotechnology 30, 204001 (2019)

391. I.B. ZeläôDovich, Sov. Phys. JETP 6, 1184 (1958)

392. C. Wood, S. Bennett, D. Cho, B. Masterson, J. Roberts, C. Tanner, C.E. Wieman, Science 275, 1759 (1997)

393. Y. Gao, C.M. Ho, R.J. Scherrer, Phys. Rev. D 89, 045006 (2014)

394. C.M. Ho, R.J. Scherrer, Phys. Lett. B 722, 341 (2013)

395. N. Papasimakis, V. Fedotov, V. Savinov, T. Raybould, N. Zheludev, Nat. Mater. 15, 263 (2016)

396. T. Kaelberer, V. Fedotov, N. Papasimakis, D. Tsai, N. Zheludev, Science 330, 1510 (2010)

397. A. Sayanskiy, M. Danaeifar, P. Kapitanova, A.E. Miroshnichenko, Adv. Opt. Mater. 6, 1800302 (2018)

398. L.-Y. Guo, M.-H. Li, X.-J. Huang, H.-L. Yang, Appl. Phys. Lett. 105, 033507 (2014)

399. V.A. Fedotov, A. Rogacheva, V. Savinov, D.P. Tsai, N.I. Zheludev, Sci. Rep. 3, 1 (2013)

400. P.C. Wu, C.Y. Liao, V. Savinov, T.L. Chung, W.T. Chen, Y.-W. Huang, P.R. Wu, Y.-H. Chen, A.-Q. Liu, N.I. Zheludev et al., ACS Nano 12, 1920 (2018)

401. N.A. Nemkov, I.V. Stenishchev, A.A. Basharin, Sci. Rep. 7, 1 (2017)

402. A.E. Miroshnichenko, A.B. Evlyukhin, Y.F. Yu, R.M. Bakker, A. Chipouline, A.I. Kuznetsov, B. Luk'yanchuk, B.N. Chichkov, Y.S. Kivshar, Nat. Commun. 6, 1 (2015)

403. M. Timofeeva, L. Lang, F. Timpu, C. Renaut, A. Bouravleuv, I. Shtrom, G. Cirlin, R. Grange, Nano Lett. 18, 3695 (2018)

404. S.-H. Kim, S.S. Oh, K.-J. Kim, J.-E. Kim, H.Y. Park, O. Hess, C.-S. Kee, Phys. Rev. B 91, 035116 (2015)

405. L. Wei, Z. Xi, N. Bhattacharya, H.P. Urbach, Optica 3, 799 (2016)

406. A.G. Lamprianidis, A.E. Miroshnichenko, Beilstein J. Nanotechnol. 9, 1478 (2018) 
407. J.S.T. Gongora, A.E. Miroshnichenko, Y.S. Kivshar, A. Fratalocchi, Nat. Commun. 8, 1 (2017)

408. I. Staude, A.E. Miroshnichenko, M. Decker, N.T. Fofang, S. Liu, E. Gonzales, J. Dominguez, T.S. Luk, D.N. Neshev, I. Brener et al., ACS Nano 7, 7824 (2013)

409. A.K. Ospanova, I.V. Stenishchev, A.A. Basharin, Laser Photonics Rev. 12, 1800005 (2018)

410. Y. Yang, V.A. Zenin, S.I. Bozhevolnyi, ACS Photonics 5, 1960 (2018)

411. T. Shibanuma, G. Grinblat, P. Albella, S.A. Maier, Nano Lett. 17, 2647 (2017)

412. G. Grinblat, Y. Li, M.P. Nielsen, R.F. Oulton, S.A. Maier, ACS Nano 11, 953 (2017)

413. S.-D. Liu, J.-L. Fan, W.-J. Wang, J.-D. Chen, Z.-H. Chen, ACS Photonics 5, 1628 (2018)

414. S.-D. Liu, Z.-X. Wang, W.-J. Wang, J.-D. Chen, Z.-H. Chen, Opt. Express 25, 22375 (2017)

415. K. Du, P. Li, K. Gao, H. Wang, Z. Yang, W. Zhang, F. Xiao, S.J. Chua, T. Mei, J. Phys. Chem. Lett. 10, 4699 (2019)

416. D.G. Baranov, R. Verre, P. Karpinski, M. Käll, ACS Photonics 5, 2730 (2018)

417. G. Grinblat, Y. Li, M.P. Nielsen, R.F. Oulton, S.A. Maier, Nano Lett. 16, 4635 (2016)

418. G. Grinblat, Y. Li, M.P. Nielsen, R.F. Oulton, S.A. Maier, ACS Photonics 4, 2144 (2017)

419. J.F. Algorri, D.C. Zografopoulos, A. Ferraro, B. García-Cámara, R. Vergaz, R. Beccherelli, J.M. Sánchez-Pena, Nanomaterials 9, 30 (2019)

420. L. Sabri, Q. Huang, J.-N. Liu, B.T. Cunningham, Opt. Express 27, 7196 (2019) 